GÖTTINGER ZENTRUM

FÜR BIODIVERSITÄTSFORSCHUNG UND ÖKOLOGIE

- Göttingen CentRe for Biodiversity ANd ECOlogy -

\title{
Evolutionary History of Snub-nosed Monkeys (Rhinopithecus spp.) and Population Genetic Structure of the Guizhou Snub-nosed Monkey (R. brelichi)
}

\author{
Dissertation zur Erlangung des Doktorgrades der \\ Mathematisch-Naturwissenschaftlichen Fakultäten der \\ Georg-August-Universität Göttingen
}

vorgelegt von

Master Degree, Medicine

Mouyu Yang

aus

Sichuan, China

Göttingen, November 2011 
Referentin/Referent: Prof. Dr. Peter M. Kappeler

Korreferentin/Korreferent: Prof. Dr. Eckhard W. Heymann

Tag der mündlichen Prüfung: 


\section{Table of Contents}

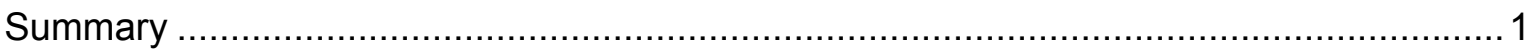

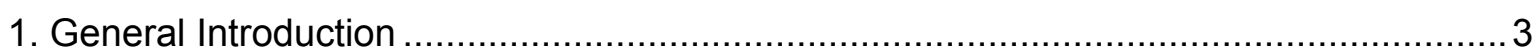

Chapter 1: Nuclear Versus Mitochondrial DNA: Evidence for Hybridization in

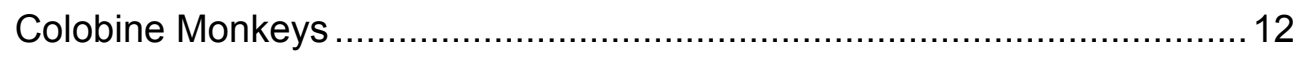

Chapter 2: The Evolutionary History of Odd-Nosed Monkeys......................................42

Chapter 3: Population Genetic Structure of Guizhou Snub-Nosed Monkeys (Rhinopithecus brelichi) as Inferred from Mitochondrial Control Region Sequences, and Comparison with $R$. roxellana and $R$. bieti .....................60

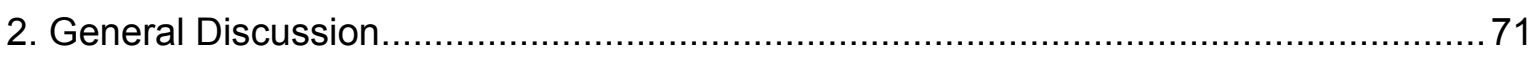

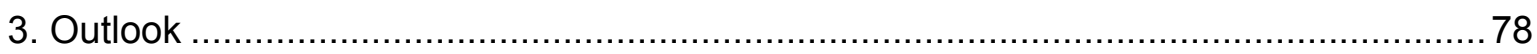

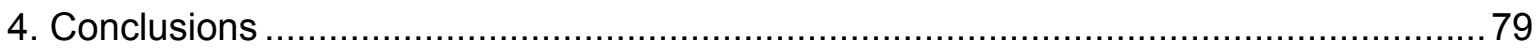

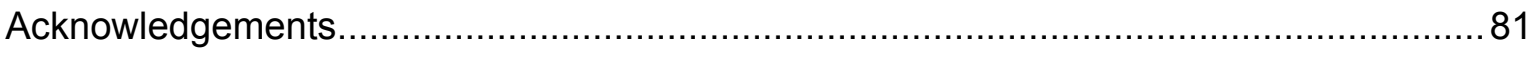

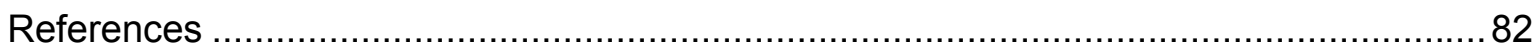

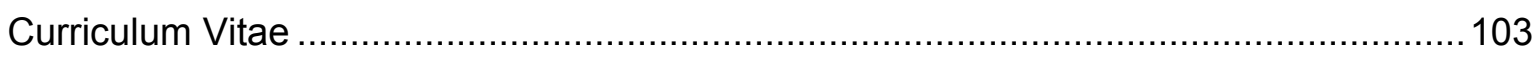

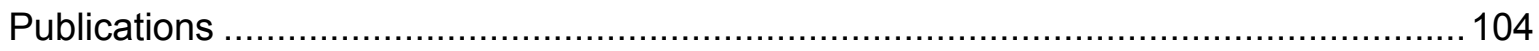

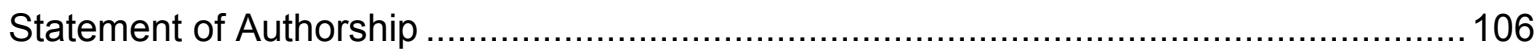




\section{Summary}

The colobines (leaf monkeys) represent a diverse group of Old World primates. They comprise ten genera with major radiations in Africa and Asia. As in most other primate radiations, recent molecular studies detected discordant gene trees depending on respective markers. Thus, the phylogeny among these genera remains debated. For the odd-nosed monkeys, one of the two major groups of Asian colobines and comprising ten species within four genera (Nasalis, Simias, Rhinopithecus and Pygathrix), the phylogenetic relationships among genera and species are also still largely unknown.

The genus Rhinopithecus represents one genus of the odd-nosed monkey group. It comprises five species, of which three are endemic to China ( $R$. roxellana, $R$. bieti, and $R$. brelichi), one occurs in northern Vietnam ( $R$. avunculus) and one in Myanmar (R. strykeri). All of them are classified as "Endangered" or "Critically Endangered" (IUCN, 2011). Among the three Chinese snub-nosed monkey species, $R$. brelichi shows the smallest wild population size and the most restricted distribution. Information about its population genetic structure is still missing.

In my thesis, I applied a top-down approach to clarify the phylogenetic relationships among all colobines genera, the phylogenetic relationships among the odd-nosed monkey species and the population genetic structure of one of the most endangered species of the colobines, the Guizhou snub-nosed monkey (R. brelichi).

The phylogenetic relationships among colobines genera are analyzed in this study by using maternal-, paternal- and biparental-inherited molecular markers (Chapter 1). The phylogenetic reconstructions from all these markers show similar results. However, a few discordances occurred, which are most likely caused by sexspecific introgression and hybridization among ancestral lineages. However, the monophyly of the odd-nosed monkeys and the relationships among genera appeared robust and was supported by the various topologies based on different genetic markers.

To further understand the phylogenetic relationships within the odd-nosed monkeys nine of the ten species were analyzed based on complete mitochondrial genome data (Chapter 2) providing the most comprehensive overview published so far. $R$. strykeri was not included in this part of my thesis, because this species was just 
recently discovered and genetic material was not available. Among the odd-nosed monkeys, the genus Rhinopithecus is most basal, whereas Pygathrix forms a sister lineage to the Nasalis + Simias clade. Within Rhinopithecus, R. roxellana $+R$. brelichi form a sister clade to $R$. bieti, while $R$. avunculus appears as the most basal taxon. For Pygathrix, the analysis supported $P$. nigripes as the basal taxon to the $P$. cinerea $+P$. nemaeus clade. The diversification of odd-nosed monkeys into genera started roughly 6.85 mya, with the major speciation events occurring during the Pleistocene.

$R$. brelichi with a maximum of 800 individuals in the wild is one of the rarest Chinese primates. However, population genetic data is crucial for assessing the persistence of a population, especially for endangered species with a small population. Previous population genetic studies focused on $R$. roxellana and $R$. bieti, but no such information was available for $R$. brelichi. Therefore, the aim of chapter 3 was an assessment of the population genetic and demographic history of $R$. brelichi in comparison with respective data from the two other Chinese species ( $R$. roxellana, $R$. bieti). The population genetic variation and the demographic history of this endangered species were assessed by using the hypervariable region I of the mitochondrial control region. All three Chinese snub-nosed monkey species showed rather low genetic diversity, while $R$. brelichi has the lowest. All tests for the demographic history proposed population equilibrium in $R$. brelichi. The low genetic diversity, the small population size and the restricted distribution imply that $R$. brelichi might be the most vulnerable species among the three Chinese snub-nosed monkeys.

Future studies should apply nuclear data to further understand the phylogenetic relationships among the odd-nosed monkeys' species and to answer questions about possible ancient hybridization events as depicted for colobines genera. For $R$. strykeri the phylogenetic position within the odd-nosed monkeys should be examined to complete the picture. Further detailed population genetic analyses for $R$. brelichi should be applied by using nuclear markers, e.g. microsatellites to get more detailed information about the genetic diversity and demographic history of $R$. brelichi. This information is also required for the two most endangered species of the genus Rhinopithecus, $R$. avunculus and $R$. strykeri. 


\section{General Introduction}

"Nothing in biology makes sense except in the light of evolution" was famously claimed by Theodosius Dobzhansky (1973). It clearly indicates that the modern evolutionary synthesis is the cornerstone of biology. Evolution is a process of change on population-level over time and space. Evolutionary research includes two central goals: 1) describe the branching order of the life history on earth, and 2) explain why species differ from one another and how the evolutionary processes occur (Whelan et al., 2001). Nei (1975) gave a clear definition of the goal of studying evolution: "understand all the processes of evolution quantitatively and be able to predict and control the future evolution of organisms".

Since Willi Hennig published his paper in 1965 "Phylogenetic Systematic", the phylogenetic tree gradually became the critical underpinning of research in all evolutionary studies (Soltis and Soltis, 2003). Phylogenetic research mainly aims to represent the evolutionary relationships among organisms (and genes) as a tree, which constitutes a phylogenetic hypothesis. Phylogenetic trees are constructed based on molecular or morphological data, or data on other traits (Edwards and Cavalli-Sforza, 1964; Stearns and Hoekstra, 2000). Phylogenies provide extremely useful information for many different fields within biology. Their paramount uses are testing hypotheses about the evolution of characters and tracking the history of any change in a specific character. Overall, phylogenies give scientists new perspectives for looking into the past (Losos, 2011). This important development has given rise to phylogenetic analyses as an instrument used for classification, identification, and naming of organisms (Edwards and Cavalli-Sforza, 1964; Wiley et al., 1991; Harvey et al., 1996). Phylogenetics is nowadays a critical important tool for investigating the evolutionary history of organisms, like J. M. Savage said "nothing in evolutionary biology makes sense except in the light of a phylogeny" (Savage, 1997 cited in Johnson et al., 2003).

Among our closest living relatives are the Old World monkeys (Cercopithecidae), one of the most diverse primate groups. Unfortunately, the evolutionary history and phylogenetic relationships among its members are not well resolved yet, thus, requiring additional phylogenetic studies. Old World monkeys comprise two subfamilies: Cercopithecinae and Colobinae (Groves, 2001). Each subfamily is clearly differentiated by distinctive morphological adaptations, mainly related to their digestive systems. The 
cercopithecines - also called cheek pouch monkeys - are predominantly frugivorous and have cheek pouches, which they use for temporarily storing food. In contrast, colobines are predominantly folivorous and they have a multi-chambered ruminant-like stomach to digest food rich in cellulose (Groves, 1970; Brandon-Jones, 1984; Davies and Oates, 1994; Groves, 2001; Fashing, 2011, Kirkpatrik, 2011).

Cercopithecines have been extensively studied in many aspects of their natural history including molecular phylogenies (e.g., Jolly, 1966; Hill, 1974; Groves, 1978; Dutrillaux, 1979; Szalay and Delson, 1979; Groves, 1987; Strasser and Delson, 1987; Disotell et al., 1992; Jablonski and Peng, 1993; Benson et al., 1998; Harris and Disotell, 1998; Morales and Melnick, 1998; Evans et al., 1999; Jolly and Whitehead, 2000; McGraw and Fleagle, 2000; Frost et al., 2003; Roos et al., 2003; Tosi et al., 2003, Roos, 2004; Tosi et al., 2004, 2005; Roos et al., 2007; Xing et al., 2007; Ziegler et al., 2007; Moulin et al., 2008; Fabre et al., 2009; Li et al., 2009; Gilbert, 2011). Less emphasis was placed on the analysis of the phylogeny of the sister taxon of the cercopithecines, the colobines. Colobines build a diverse group of monkeys (Oates, 1994) with ongoing disputes about phylogenetic relationships among its members (Groves, 1989; Jablonski, 1998c; Jablonski, 1999; Stewart and Disotell, 1999; Zhang and Ryder, 1999) and their molecular phylogeny has only recently been studied (Zhang and Ryder, 1998; Roos, 2004; Sterner et al., 2006; Whittaker et al., 2006; Roos et al., 2007; Osterholz et al., 2008; Roos et al., 2008; Ting, 2008; Ting et al., 2008; Karanth, 2010; Md Zain et al., 2010; Meyer et al., 2011). One reason is probably that most of these species are difficult to keep in captivity and obtaining samples from them in the wild is due to their mainly arboreal life style more difficult than from cercopithecines (Zhang and Ryder, 1998; Xing et al., 2005).

Traditionally, colobines have been divided into an African and Asian group based on both morphological traits and geographical distribution (Delson, 1975). Both taxa are believed to form reciprocally monophyletic groups (Napier and Napier, 1967; Szalay and Delson, 1979; Davies and Oates, 1994; Groves, 2001), though paraphyly has also been proposed (Groves, 1989; Jablonski, 1998c). African colobines are distributed in many parts of Sub-Saharan Africa, but are predominantly found in the western and central rain humid forests. They are arboreal and share traits such as a reduced or missing pollex, mid-tarsal shortening, and some other postcranial and dental features (Szalay and Delson, 1979; Strasser and Delson, 1987; Falk, 2000; 
Groves, 2001). Phylogenetic hypotheses for the African colobines have been developed based on pelage coloration and anatomical features (Kingdon, 1997; Groves, 2001; Grubb et al., 2003; Groves, 2007). Traditionally, all African colobines were classified as members of the genus Colobus (Szalay and Delson, 1979). Verheyen (1962) and Davies and Oates (1994) split the African colobines into two genera, Procolobus and Colobus, while some authors also separate red colobus monkeys from Procolobus as a distinct genus, Piliocolobus (Struhsaker, 1981; Groves, 2001; Grubb et al., 2003; Groves, 2007). The Piliocolobus + Procolobus clade is regarded as sister group of Colobus (Hill, 1952; Kuhn, 1972; Napier, 1985; Strasser and Delson, 1987; Ting, 2008).

Asian colobines are distributed throughout Southeast Asia, including Southern China, the Indian subcontinent, Indochina and Sundaland. They occupy a variety of habitats ranging from tropical rainforests to semi-desert conditions (Bennet and Davies, 1994). They are predominantly arboreal, but under certain conditions, such as in the semi-desert of north-western India, they are almost entirely terrestrial (Hanuman langurs of Jodhpur, Vogel, 1976). Traits such as a shorter face and the presence of a suborbital fossa distinguish them from the African species. Although the Asian colobines have somewhat larger pollexes than the African species, their pollexes are still reduced compared to those of the cercopithecines (Falk, 2000; Groves, 2001). Asian colobines are more diverse than the African colobines (Oates and Davies, 1994) and they comprise the langur/leaf monkey group with three genera (Presbytis, Semnopithecus, Trachypithecus) and the odd-nosed monkey group with four genera (Nasalis, Simias, Rhinopithecus, Pygathrix) (Jablonski and Peng, 1993; Jablonski, 1998c; Falk, 2000; Groves, 2001; Brandon-Jones et al., 2004; Groves, 2007; Osterholz et al., 2008).

The taxonomy of langurs and leaf monkeys is still disputed and contradicting. In the most recent overview (IUCN, 2011), Trachypithecus is acknowledged consisting of 16 species, Semnopithecus including eight species, and Presbytis comprising 11 species. Similarly to their taxonomy, the phylogenetic relationships among the langurs and leaf monkeys are also disputed. All langurs and leaf monkeys have been originally classified in the single genus Semnopithecus or Presbytis (Reichenbach, 1862; Napier and Napier, 1967; Groves, 1970; Delson, 1975). Brandon-Jones (1984) separated Semnopithecus from Presbytis and established Trachypithecus as a morphological 
subgenus of the former based on the overall appearance. Based on neonatal coloration and cranial morphology, Pocock (1935) divided langurs and leaf monkeys into the three genera Semnopithecus, Trachypithecus, and Presbytis. Hill (1934) and added Kasi as a fourth genus. However, recent classification of the langurs has been commonly accepted as consisting of three genera: Presbytis, Trachypithecus and Semnopithecus (Weitzel et al., 1988; Groves, 1989; Davies and Oates, 1994; Groves, 2001; BrandonJones et al., 2004; Osterholz et al., 2008; Karanth, 2010). Phylogenetic studies now arrange Semnopithecus with Trachypithecus to the exclusion of Presbytis (BrandonJones, 1984; Strasser and Delson, 1987; Brandon-Jones, 1995; Groves, 2001; Osterholz et al., 2008; Ting et al., 2008) or place Trachypithecus and Presbytis as sister taxa to the exclusion of Semnopithecus (Sterner et al., 2006).

Odd-nosed monkeys comprise the genera Rhinopithecus, Pygathrix, Nasalis and Simias. All are characterised by particular modifications to their noses (Groves 2001). The noses of the Nasalis/Simias group are entire. Their face is long and narrow and the inter-orbital pillar looks unusually narrow. Proboscis monkeys (Nasalis) have a long and narrow skull with elongated noses (in particular adult males) and strong supraorbital ridges. This genus comprises only one species ( $N$. larvatus), which lives mainly in mangrove and riverine forests on Borneo. Pig-tailed monkeys (Simias) have smaller noses than Nasalis and a very short upturned, curly and nearly hairless tail. The single species $S$. concolor is endemic to the Mentawai Islands, west of Sumatra. The Pygathrix/Rhinopithecus group shows flaps of skin on the upper margins of the nostrils and their nasal bones are reduced. Their face looks short and wide. Douc langurs (Pygathrix) have a short and wide facial skeleton, with strong supra-orbital ridges and a broad inter-orbital pillar. It comprises three species, red-shanked doucs ( $P$. nemaeus), gray-shanked doucs ( $P$. cinerea) and black-shanked doucs ( $P$. nigripes). Doucs are distributed through parts of Vietnam, Laos and Cambodia, east of the Mekong River.

Snub-nosed monkeys (Rhinopithecus, Fig. 1) display a more prognathous and wider facial skeleton and stronger supra-orbital torus than Pygathrix and the palate is longer. This genus comprises five species: the Tonkin snub-nosed monkey $(R$. avunculus), the Myanmar snub-nosed monkey ( $R$. strykeri), the golden or Sichuan snub-nosed monkey ( $R$. roxellana), the gray or Guizhou snub-nosed monkey $(R$. brelichi), and the black or Yunnan snub-nosed monkey (R. bieti) (Groves, 2001; Roos et al., 2007; Geissmann et al., 2011; IUCN, 2011). 


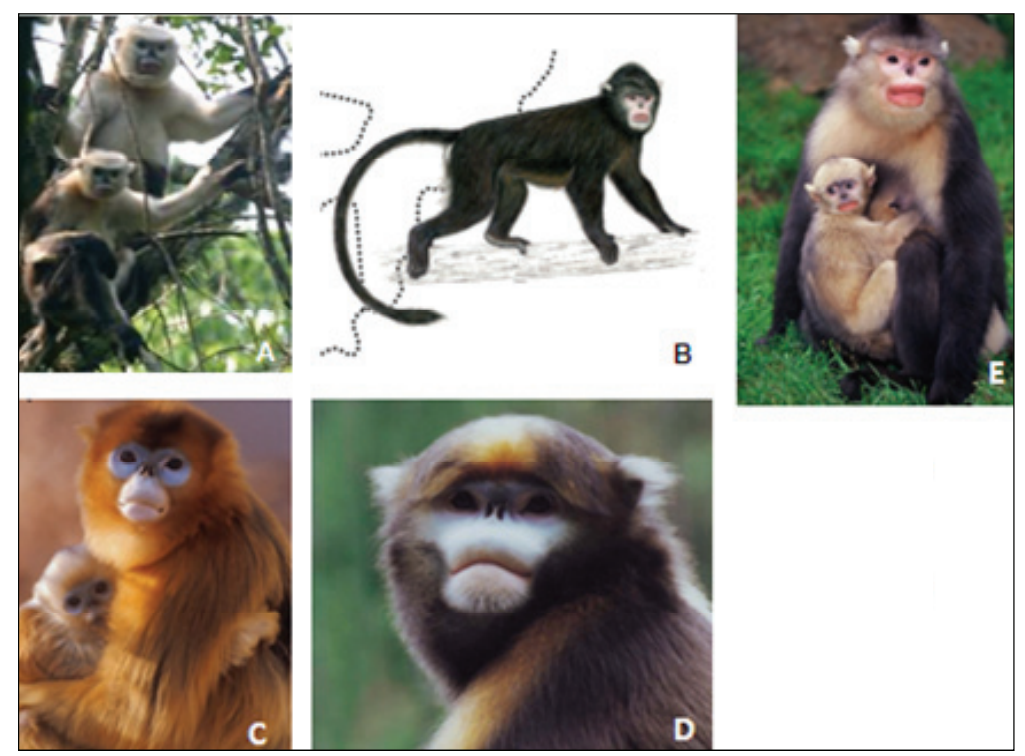

Figure 1: The five snub-nosed monkey species. A) $R$. avunculus; B) $R$. strykeri; C) $R$. roxellana; D) R. brelichi; E) R. bieti (photographs and drawings from Dong Thanh Hai, Primate Conservation INC.; Stephen Nash, Conservation International; Heather Angel, Naturalvisions.co.uk; Yeqin Yang, Fanjingshan National Nature Reserve; China Kunming animal institution).

Although a common origin of odd-nosed monkeys has been supported by recent genetic analyses (Sterner et al., 2006; Osterholz et al., 2008; Ting et al., 2008; Li et al., 2011; Perelman et al., 2011), debates on the phylogenetic relationships among genera and species still continue. Based on morphological or molecular data, previous studies proposed several phylogenetic hypotheses: (1) a sister taxon relationship between Rhinopithecus and Pygathrix (Delson, 1975; Napier, 1985; Jablonski and Peng, 1993; Oates et al., 1994; Wang et al., 1997; Groves, 2001; Li et al., 2004); (2) a sister taxon relationship between Pygathrix and Nasalis to the exclusion of Rhinopithecus (Jablonski, 1998c); (3) a sister taxon relationship between Rhinopithecus and Nasalis (Zhang and Ryder, 1998); (4) a sister taxon relationship between Simias and Nasalis (Whittaker et al., 2006); and (5) a basal position of Simias among Asian colobines (Jablonski, 1998c).

The monophyly of odd-nosed monkey genera is generally accepted, but Peng et al. (1993) suggested a paraphyly of Rhinopithecus and placed $R$. avunculus as sister taxon to Pygathrix. Within Pygathrix, $P$. nigripes is basal to a clade consisting of $P$. cinerea and P. nemaeus (Roos and Nadler, 2001; Roos, 2004; Roos et al., 2007). In contrast, the phylogenetic relationships among the five Rhinopithecus species are 
debated. Jablonski and Peng (1993) pointed out that $R$. roxellana clusters together with the $R$. bieti $+R$. brelichi clade. A three-way relationship between R. bieti, R. roxellana and $R$. avunculus was proposed by Zhang and Ryder (1998). This hypothesis was supported by molecular studies of Roos (2004), Roos et al. (2007) and Li et al. (2004). In a recent study by $\mathrm{Li}$ et al. (2011), $R$. avunculus appeared as the sister lineage to the $R$. bieti $+R$. roxellana clade. Although $R$. strykeri was not included in all these studies, this species resembles $R$. bieti in many morphological attributes suggesting a sister taxon relationship of the two species (Geissmann et al., 2011).

Although the phylogenetic relationships within the Asian colobines became clearer due to a number of recent molecular studies, several questions remain especially in light of the observed incongruences found among various gene tree phylogenies (Sterner et al., 2006; Karanth et al., 2008; Osterholz et al., 2008; Ting et al., 2008). Therefore, the first aim of my study is to clarify the phylogenetic relationships among the four odd-nosed monkey genera and respective species.

Many of the Asian colobines are endangered including all snub-nosed monkey species (IUCN, 2011). The ultimate interest of conservation is the preservation of genetic diversity (Avise and Hamrick, 1995). The degree of genetic diversity within a population can have impacts on the long-term survival of the respective population, especially in case of rare and endangered species (Frankham et al., 2004). Molecular methods have been successfully applied in conservation biology, in particular on the population genetic level. Information about the genetic diversity within a population often provides hints for the management of such populations.

Fossils records of snub-nosed monkeys indicate that they were widely distributed in China and Vietnam during the Pleistocene ( $\mathrm{Li}$ et al., 2002). The contemporary highly fragmented ranges of snub-nosed monkeys most likely result from environmental changes in the Holocene (Pan and Jablonski, 1987; Pan, 1995), and from increasing human activities during the last centuries. Suitable habitats for snubnosed monkeys were heavily reduced and their populations survived only in restricted areas (Jablonski, 1998b; Li et al., 2002). Currently, R. avunculus occurs in tropical monsoon forests in Tuyen Quang, Bac Kan, Thai Nguyen and Ha Giang provinces of northern Vietnam at elevations of 200 - 1,200 m (Kirkpatrick, 1995; Nguyen, 2000; Groves, 2001; Nadler et al., 2003; Dong and Boontatana, 2006; IUCN, 2011) (Fig. 2). Its population comprises roughly 200 individuals (Covert et al., 2008; Mittermeier et al., 
2009; IUCN, 2011). R. strykeri has been recently discovered in Kachin state in northeastern Myanmar where it inhabits mixed temperate and conifer forests at 1,720 3,190 m with an estimated population size of 260 - 330 individuals (Geissmann et al., 2011). The remaining three species are endemic to mountainous regions of southwestern China. R. roxellana occupies deciduous broadleaf, conifer and mixed forests at an elevation of 1,200 - 3,000 $\mathrm{m}$ in the provinces of Sichuan, Hubei, Shaanxi and Gansu and its population size is approximately 15,000 individuals (IUCN, 2011). R. bieti inhabits evergreen forests composed primarily of conifers, evergreen oak in Yunnan and Tibet at 2,700 - 4,600 m. Its population comprises about 2,000 individuals (IUCN, 2011). R. brelichi lives in mixed deciduous and evergreen broadleaf forests in Guizhou at 1,500 - 2,200 $\mathrm{m}$ and its population comprises 750 - 800 individuals (Yang et al., 2002; IUCN, 2011). All species are today classified as "Endangered" or even "Critically Endangered" (IUCN, 2011).

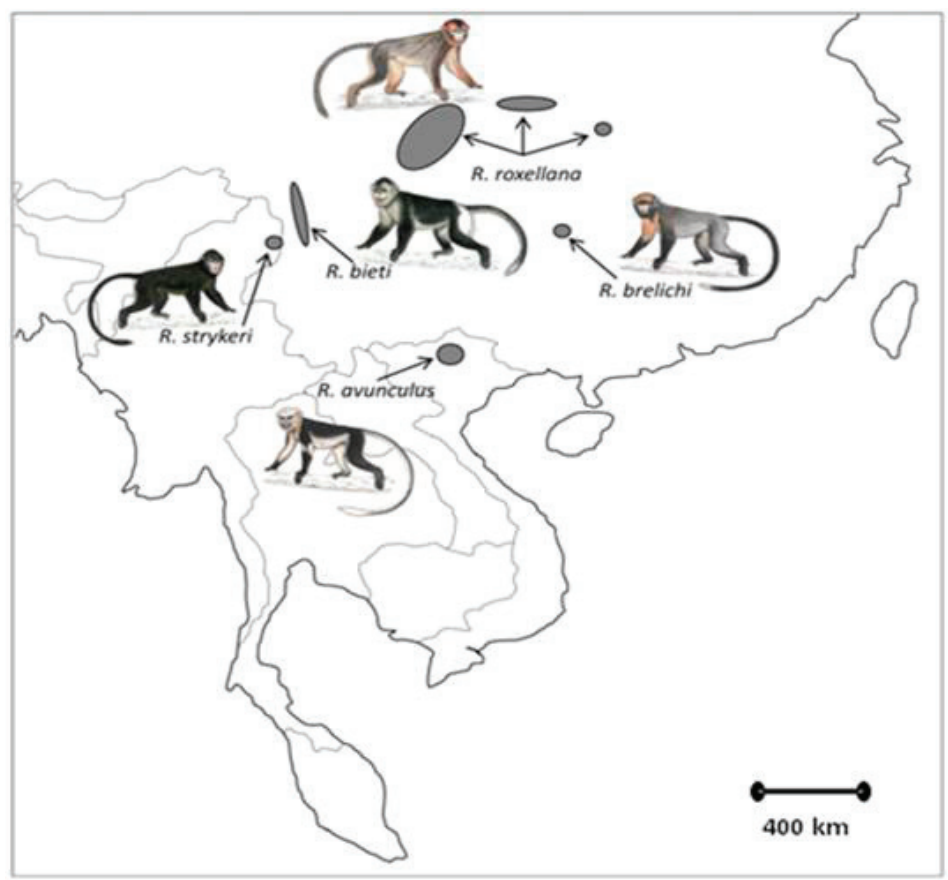

Figure 2: Map of the northern part of Southeast Asia indicating the approximate distributions of the five snub-nosed monkey species. Illustrations of monkeys by Stephen Nash, Conservation International.

The Guizhou snub-nosed monkey (R. brelichi) (Fig. 3) has the most restricted distribution and comprises the smallest population compared to the other two Chinese 
snub-nosed monkey species. It occurs only in evergreen and deciduous broadleaf forests in a strictly protected core area of about $260 \mathrm{~km}^{2}$ of the Fanjingshan National Nature Reserve, a small area of the Wuling Mountains in Guizhou province (Bleisch and Xie, 1998; Groves, 2001; Yang et al., 2002; Wu et al., 2004; MacKinnon, 2008; IUCN, 2011). Additionally, in a inter-specific comparison among the three Chinese snub-nosed monkey species indications were found that $R$. brelichi has the slowest population recovery capability due to a slower life-history (female age at first birth: $R$. brelichi 8-9 years, $R$. roxellana and $R$. bieti 5-6 years; longer inter-birth interval: $R$. brelichi: 3 years, $R$. roxellana and R. bieti 2 years (Ji et al., 1998; Zhang et al., 2000; Liang et al., 2001; Cui et al., 2006; Qi et al., 2008; Yang et al., 2009).

Population genetic data for $R$. brelichi are not available. Therefore, I did a detailed population genetic study of $R$. brelichi and compared it with already existing data for the other two Chinese snub-nosed monkey species (Li et al., 2001; Li et al., 2003; Li et al., 2007; Liu et al., 2007). The second aim of my thesis is to analyze the level of genetic diversity and demographic history of the wild population of $R$. brelichi.

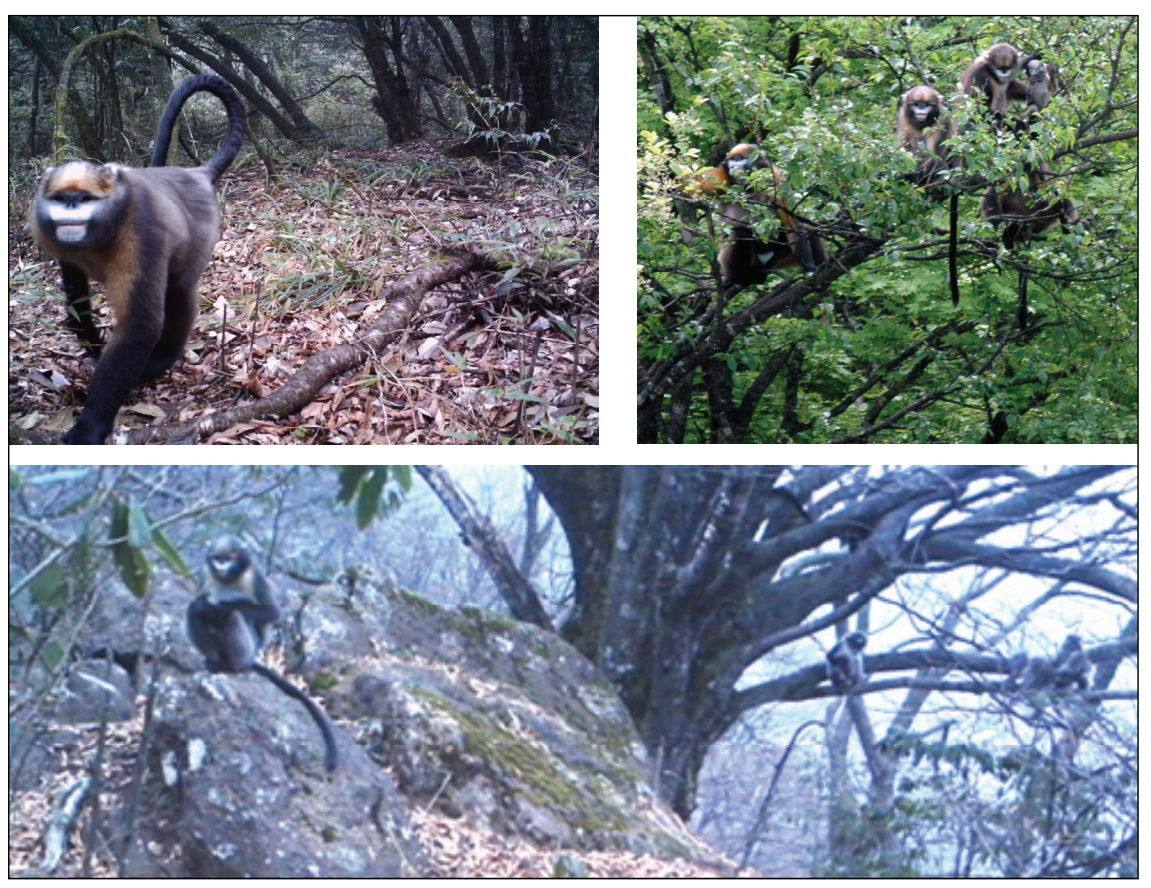

Figure 3: Guizhou snub-nosed monkeys in the Fanjingshan National Nature Reserve (Photographs taken with camera traps). 


\section{Aim and content of this thesis}

The first aim of my study was to elucidate the phylogenetic relationships among odd-nosed monkey genera and among respective species. The second aim was to analyze the population history and genetic diversity of $R$. brelichi. I organized my study in a top down manner, i.e. from the higher taxonomic levels to the species and population levels.

Chapter 1: On the first level we analyzed the evolutionary history of the subfamily Colobinae, in particular the phylogenetic relationships among the odd-nosed monkey genera. To obtain a better understanding of the evolutionary history of the oddnosed monkey group, we analyzed sequence data from mitochondrial genomes and 12 nuclear loci (in total $\sim 30,000$ bp per genus), and combined it with the presence / absence patterns of mobile elements. (Roos et al. 2011. Nuclear versus mitochondrial DNA: evidence for hybridization in colobine monkeys. BMC Evolutionary Biology 11: 77).

Chapter 2: In a second step, we determined the phylogenetic relationships among nine of the ten odd-nosed monkey species using complete mitochondrial genome sequences. The obtained phylogeny is robust and provides new insights into the evolutionary history of the odd-nosed monkey group. (Yang et al. in press. The evolutionary history of odd-nosed monkeys. In: Tan C, Grueter C, Wright B, editors. Odd-nosed Monkeys: Recent Advances in the Study of the Forgotten Colobines).

Chapter 3: On the third level, my aim was to compare the genetic diversity of the three Chinese snub-nosed monkey species based on mitochondrial data (hypervariable region I of the control region). Analyses on level three are also designed to provide useful information for conservation management decisions. Here, I provide a comprehensive overview over the population genetic structure and the population history of the three Chinese Rhinopithecus species. (Yang et al. 2011. Population genetic structure of Guizhou snub-nosed monkeys (Rhinopithecus brelichi) as inferred from mitochondrial control region sequences, and comparison with $R$. roxellana and $R$. bieti. Doi 10.1002/ajpa.21618 
Chapter 1

\section{Nuclear Versus Mitochondrial DNA: \\ Evidence for Hybridization in Colobine Monkeys}

Published in:

BMC Evolutionary Biology 11: 77 


\title{
Nuclear versus mitochondrial DNA: evidence for hybridization in colobine monkeys
}

\author{
Christian Roos ${ }^{1,2^{*}+}$, Dietmar Zinner ${ }^{3+}$, Laura S Kubatko ${ }^{4}$, Christiane Schwarz ${ }^{1}$, Mouyu Yang ${ }^{1}$, Dirk Meyer ${ }^{5}$, \\ Stephen D Nash ${ }^{6}$, Jinchuan Xing ${ }^{7}$, Mark A Batzer ${ }^{8}$, Markus Brameier ${ }^{1}$, Fabian H Leendertz ${ }^{9}$, Thomas Ziegler ${ }^{5}$, \\ Dyah Perwitasari-Farajallah ${ }^{10}$, Tilo Nadler ${ }^{11}$, Lutz Walter ${ }^{1,2}$, Martin Osterholz $z^{1,12^{*}}$
}

\begin{abstract}
Background: Colobine monkeys constitute a diverse group of primates with major radiations in Africa and Asia. However, phylogenetic relationships among genera are under debate, and recent molecular studies with incomplete taxon-sampling revealed discordant gene trees. To solve the evolutionary history of colobine genera and to determine causes for possible gene tree incongruences, we combined presence/absence analysis of mobile elements with autosomal, $\mathrm{X}$ chromosomal, $\mathrm{Y}$ chromosomal and mitochondrial sequence data from all recognized colobine genera.
\end{abstract}

Results: Gene tree topologies and divergence age estimates derived from different markers were similar, but differed in placing Piliocolobus/Procolobus and langur genera among colobines. Although insufficient data, homoplasy and incomplete lineage sorting might all have contributed to the discordance among gene trees, hybridization is favored as the main cause of the observed discordance. We propose that African colobines are paraphyletic, but might later have experienced female introgression from Piliocolobus/Procolobus into Colobus. In the late Miocene, colobines invaded Eurasia and diversified into several lineages. Among Asian colobines, Semnopithecus diverged first, indicating langur paraphyly. However, unidirectional gene flow from Semnopithecus into Trachypithecus via male introgression followed by nuclear swamping might have occurred until the earliest Pleistocene.

Conclusions: Overall, our study provides the most comprehensive view on colobine evolution to date and emphasizes that analyses of various molecular markers, such as mobile elements and sequence data from multiple loci, are crucial to better understand evolutionary relationships and to trace hybridization events. Our results also suggest that sex-specific dispersal patterns, promoted by a respective social organization of the species involved, can result in different hybridization scenarios.

\section{Background}

With more than 50 species and due to some ecological adaptations, such as a ruminant-like chambered stomach to digest food rich in fiber, the Old World monkey subfamily Colobinae represents a diverse and enigmatic group of primates $[1,2]$. Colobines are predominantly arboreal and occur in forest and woodland habitats. They have experienced two major radiations, one in Africa with the genera Procolobus, Piliocolobus and

\footnotetext{
*Correspondence: croos@dpz.eu; mosterh@gwdg.de

+ Contributed equally

'Primate Genetics Laboratory, German Primate Center, Kellnerweg 4, 37077 Göttingen, Germany

Full list of author information is available at the end of the article
}

Colobus, and a second in South and Southeast Asia comprising the langur genera Semnopithecus, Trachypithecus and Presbytis, and the odd-nosed monkey genera Rhinopithecus, Pygathrix, Nasalis and Simias [2]. However, their phylogenetic relationships are disputed [3-7], and recent molecular studies detected substantial gene tree discordance [8-10].

Traditionally, African and Asian genera are believed to form reciprocally monophyletic groups $[1,2,11,12]$, though paraphyly has also been proposed [3-5]. Molecular investigations clearly confirm a common origin of Asian colobines and the odd-nosed monkey group [8-10], but evidence for monophyly of the langur group as well as for African colobines is still lacking.
C Biomed Central

C 2011 Roos et al; licensee BioMed Central Ltd. This is an Open Access article distributed under the terms of the Creative Commons Attribution License (http://creativecommons.org/licenses/by/2.0), which permits unrestricted use, distribution, and reproduction in any medium, provided the original work is properly cited. 
Moreover, nuclear and mitochondrial data indicate conflicting relationships among langur genera, and between langurs and the odd-nosed monkeys [8-10]. While nuclear data consistently link Semnopithecus and Trachypithecus to the exclusion of all other Asian colobines $[9,10]$, mitochondrial data either do not resolve these relationships [9] or suggest a clade consisting of Presbytis and Trachypithecus [8].

Incongruent phylogenetic relationships among genes, like those detected among colobines are common in phylogenetic studies and could be explained by homoplasy, insufficient data, nucleotide composition, differential lineage sorting, or hybridization [13-21]. To ascertain which of these possibilities are responsible for the incongruence, information from various independent molecular loci can be helpful [22]. To date, only mitochondrial and $\mathrm{X}$ chromosomal data as well as presence/ absence information of mobile elements, all based on an incomplete taxon sampling, are available for comparative phylogenetic studies in colobines [8-10,23]. Among all marker systems, mobile element insertions are a promising tool to uncover phylogenetic relationships among colobine genera. Compared to sole sequence data, mobile elements such as Short Interspersed Elements (SINEs) and Long Interspersed Elements (LINEs) exhibit advantages which make them ideal markers for phylogenetic reconstructions (for review see [24-30]). Accordingly, mobile elements are successfully applied in numerous primate phylogenetic studies [9,28,31-39].

In our study, we examined the presence/absence pattern of mobile elements and compared the inferred phylogeny with those derived from mitochondrial and nuclear sequence data (in total $\sim 30,000$ bp per genus). We extended available $\mathrm{X}$ chromosomal and mitochondrial genome data, and sequenced de novo five autosomal loci that map to different human chromosomes, and six $\mathrm{Y}$ chromosomal loci from all ten colobine genera. By combining results from different marker systems, we provide detailed insights into the evolutionary and biogeographic history of colobine monkeys, and show that different hybridization mechanisms might have been involved during the colobine radiation.

\section{Results}

\section{Nuclear phylogeny}

Eighty-three mobile elements are phylogenetically informative for colobines (Figure 1A, Additional file 1). Each of the following clades is strongly supported by at least five integrations: all colobines (clade I [A-I]), Asian colobines (A-IV), odd-nosed monkeys (A-VII), Trachypithecus and Semnopithecus (A-V), and Nasalis and Simias (A-IX). Three integrations were found in Piliocolobus and Procolobus and all Asian colobines (A-II), but not in Colobus. Two insertions suggested a sister grouping of Procolobus and Piliocolobus (A-III), Presbytis and the odd-nosed monkeys (A-VI), and a basal position of Rhinopithecus among the latter (A-VIII). Based on maximum-parsimony (MP) bootstrap analysis, most relationships were strongly supported ( $\geq 95 \%)$. Only the Piliocolobus/Procolobus (A-III), Presbytis/odd-nosed monkey (A-VI), and Pygathrix/Nasalis/Simias (A-VIII) clades gained relatively weak bootstrap values (86\%). Based on alternative tree topology tests, different positions of the Piliocolobus/Procolobus clade and Presbytis among colobines were not rejected $(P>0.05)$, while relationships other than the most likely one were significantly rejected for all other taxa $(P<0.001, P<0.05)$ (Additional file 2).

Next, we performed phylogenetic analyses based on the concatenated nuclear sequence dataset, including five autosomal loci, six Y chromosomal loci and a fragment of the X chromosomal Xq13.3 region (see Methods for detailed locus description). We combined all nuclear sequence data, because heuristic search methods for individual loci produced no conflicting relationships (Additional file 3), and partition homogeneity tests revealed no significant difference in their evolutionary history (Y chromosomal loci combined: $P=0.2939$; autosomal loci combined: $P=0.1543$; all nuclear loci combined: $P=0.3559$ ). Nucleotide composition of studied species was similar (Additional file 4). Phylogenetic reconstructions yielded identical and significantly supported branching patterns irrespectively of the applied algorithm (MP, neighbor-joining [NJ], maximum-likelihood [ML], Bayesian) (Figure 1B, for a phylogram see Additional file 5). Only the Pygathrix/Nasalis/Simias (BVIII) clade had lower support values (MP: 93\%, NJ: 80\%, but ML: 98\%, Bayesian posterior probabilities [PP]: 1.0). The resultant tree topology was mainly congruent with the mobile element-based phylogeny, but two cases of incongruence were obvious. First, in the nuclear sequence-based phylogeny, African (B-II) and Asian (BIV) colobine genera formed reciprocally monophyletic clades and second, Presbytis represented a sister lineage to the other Asian genera (B-V). According to alternative tree topology tests (Additional file 2), paraphyly of African colobines with Piliocolobus/Procolobus being closer related to Asian colobines than to Colobus as well as various alternative positions of Presbytis among Asian colobines were not rejected $(P>0.05)$. However, affiliations of Presbytis to either Semnopithecus or Trachypithecus were rejected $(P<0.001)$.

Estimated divergence ages from the combined nuclear dataset (Table 1) and single loci (Additional file 6), both based on an a-priori fixed tree topology as obtained from mobile elements, differed slightly, most likely due to the general low variability in the studied loci (Additional file 7). However, estimates were in the same range suggesting that loci evolve at similar evolutionary rates (Additional file 8). 


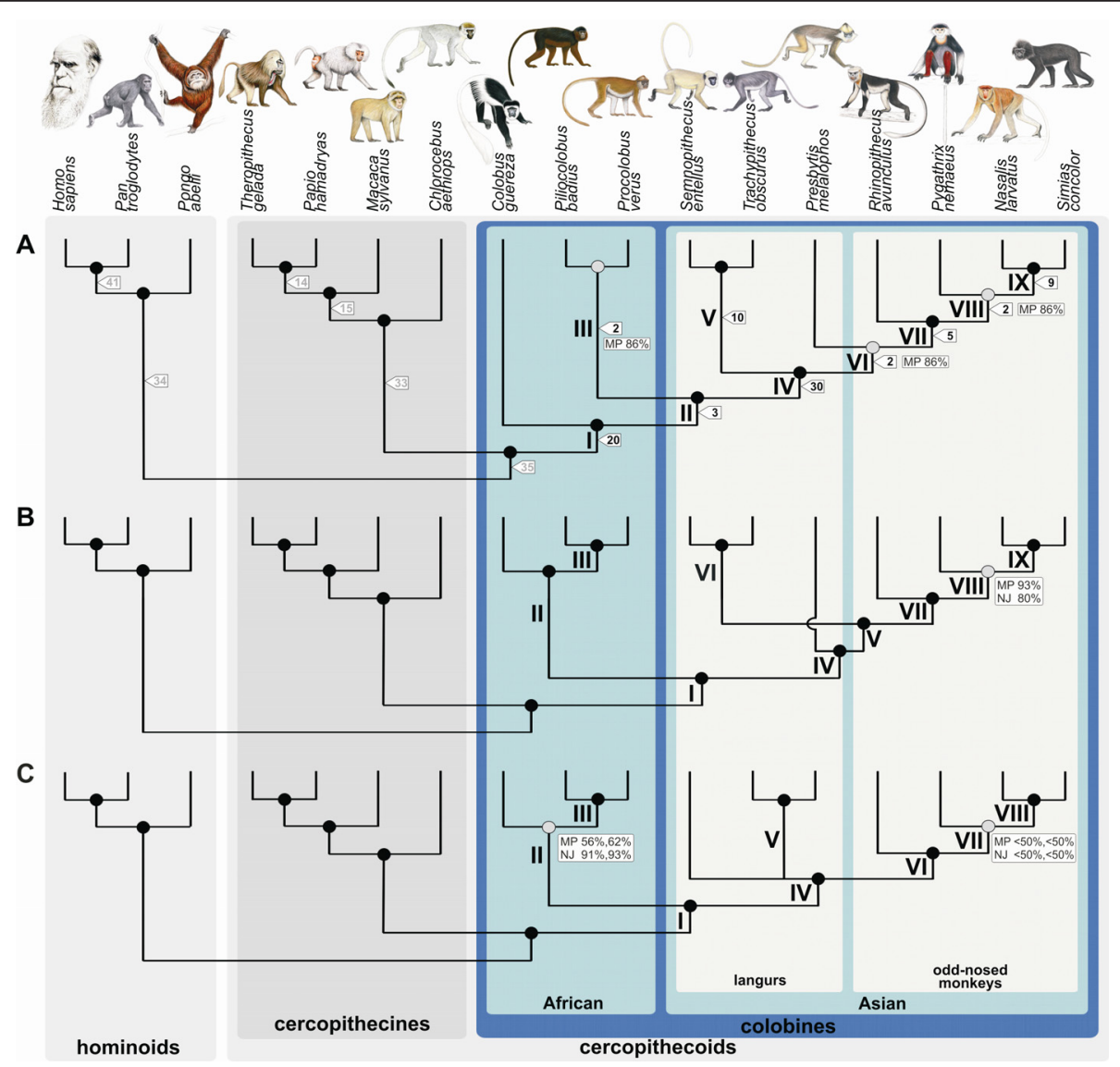

Figure 1 Phylogenetic relationships among colobine and outgroup genera as inferred from different datasets. Panels refer to insertions of mobile elements (A), combined nuclear sequence data (B), and mitochondrial genome data (C). Roman numbers are used as branch identifiers and are discussed in the text. In A, numbers in flags represent the number of available mobile elements (black: colobine markers, grey: non-colobine markers). In B and C, all nodes are significantly supported by ML and Bayesian reconstructions ( $\geq 95 \%, 1.0)$. Black and grey dots on nodes indicate high ( $\geq 95 \%$ ) and lower ( $<95 \%$ ) branch support as obtained from MP (in A-C) and NJ (in B and C) reconstructions, respectively. Bootstrap values $<95 \%$ are presented at respective nodes. In C, first and second values refer to those obtained from reconstructions using datasets mtDNA1 and mtDNA2, respectively.

According to our nuclear estimates, Colobus and Piliocolobus/Procolobus successively split off from Asian genera 10.93 million years ago (mya) and 10.73 mya, respectively (for 95\% highest posterior densities see Table 1). The latter two separated 6.92 mya. In Asia, an initial split occurred 8.12 mya and led to a clade consisting of Trachypithecus and Semnopithecus, and a group containing Presbytis and the odd-nosed monkeys. Among the latter, Presbytis diverged 7.96 mya and the odd-nosed monkeys began differentiating 6.43 mya. The most recent splits among Asian genera occurred between Trachypithecus and Semnopithecus (2.56 mya) and between Nasalis and Simias (1.06 mya).

\section{Mitochondrial phylogeny}

Mitochondrial and nuclear datasets were not combined, because the partition homogeneity test suggested that both track different evolutionary histories $(P=0.0002)$. Thus, mitochondrial sequence data were analyzed separately. For both alignments (mtDNA1, mtDNA2; for details about alignments see Methods), we observed a major shift in nucleotide composition between colobine and non-colobine representatives (Additional file 4). Both alignments produced identical and significantly supported branching patterns among genera (Figure 1C, for a phylogram see Additional file 5). Only the Pygathrix/Nasalis/Simias (CVII) and African colobine (C-II) clades gained low MP $(<50 \%,<50 \%, 56 \%, 62 \%)$ and NJ $(<50 \%,<50 \%, 91 \%, 93 \%)$ bootstrap values, but ML and Bayesian reconstructions provided strong support for both nodes (96\%, 100\%; 1.0, 1.0). In principal, the tree topology was identical to those obtained from mobile elements and nuclear sequence data. However, as in the nuclear sequence tree, mitochondrial data suggested African (C-II) and Asian (C-IV) colobines as reciprocal monophyletic clades. Moreover, Asian colobines further diverged into a lineage leading to the odd-nosed monkeys (C-VI), a lineage comprising 
Table 1 Estimation of divergence ages in mya ( $95 \%$ highest posterior density)

\begin{tabular}{lcc}
\hline node & nuclear DNA & mitochondrial DNA \\
\hline cercopithecoids - hominoids & $24.39(22.44-26.47)$ & $23.73(21.88-25.94)$ \\
Pongo - Homo/Pan & $13.89(12.80-14.95)$ & $13.58(12.51-14.64)$ \\
Homo - Pan & $6.39(5.85-7.01)$ & $6.18(5.62-6.70)$ \\
cercopithecines - colobines & $15.50(14.45-16.56)$ & $15.92(14.11-17.79)$ \\
Cholorocebus - other cercopithecines & $9.47(7.52-11.57)$ & $10.56(8.78-12.29)$ \\
Macaca - Papio/Theropithecus & $6.59(5.12-8.27)$ & $8.55(6.82-10.03)$ \\
Papio - Theropithecus & $3.80(3.20-4.38)$ & $3.97(3.39-4.46)$ \\
Colobus - other colobines (A-I) & $10.93(9.60-12.31)$ & - \\
Piliocolobus/Procolobus - Asian colobines (A-II) & $10.73(9.38-12.04)$ & - \\
African - Asian colobines (C-I) & - & $10.90(9.34-12.44)$ \\
Colobus - Piliocolobus/Procolobus (C-II) & - & $8.47(6.83-9.88)$ \\
Piliocolobus - Procolobus (A-III, C-III) & $6.92(4.38-9.35)$ & $6.58(4.99-8.04)$ \\
Asian colobines (A-IV, C-IV) & $8.12(7.14-9.16)$ & $8.91(7.43-10.23)$ \\
Trachypithecus - Semnopithecus (A-V) & $2.56(1.25-4.22)$ & - \\
Presbytis - odd-nosed monkeys (A-VI) & $7.96(6.93-8.95)$ & - \\
Presbytis - Trachypithecus (C-V) & - & $7.45(5.88-8.86)$ \\
odd-nosed monkeys (A-VIII, C-VI) & $6.43(5.03-7.75)$ & 6.91 (5.60-8.20) \\
Pygathrix - Nasalis/Simias (A-VIII, C-VII) & $5.66(4.22-7.01)$ & $6.23(5.11-7.38)$ \\
Nasalis - Simias (A-IX, C-VIII) & $1.06(0.44-1.81)$ & $1.88(1.21-2.45)$ \\
\hline
\end{tabular}

Trachypithecus and Presbytis $(\mathrm{C}-\mathrm{V})$, and finally a lineage with solely Semnopithecus, while the relationships among these three lineages remained unresolved.

According to alternative tree topology tests, paraphyly of African colobines with Piliocolobus/Procolobus being closer related to Asian colobines than to Colobus was rejected $(P<0.001$, Additional file 2). Among Asian colobines, relationships in which Trachypithecus and Presbytis do not form a monophyletic clade were also rejected $(P<0.001, P<0.05)$, as well as a close relationship of Trachypithecus and Semnopithecus $(P<0.01)$. In contrast, different positions of Semnopithecus among Asian colobines were similarly likely $(P>0.05)$.

Divergence age estimates from mitochondrial data were similar to nuclear estimates in case where identical branching patterns were obtained (Table 1). According to mitochondrial data, African and Asian colobine lineages were separated 10.90 mya. In Africa, Colobus represents the first split ( 8.47 mya), followed by the divergence of Piliocolobus and Procolobus (6.58 mya). The major Asian split leading to the three lineages Semnopithecus, Trachypithecus/Presbytis and the odd-nosed monkeys occurred 8.91 mya. Trachypithecus diverged from Presbytis 7.45 mya. The diversification of oddnosed monkeys into genera started 6.91 mya and ended with the split between Nasalis and Simias 1.88 mya.

\section{Inferring hybridization in the presence of incomplete lineage sorting}

To assess the possible reasons for the incongruence between the nuclear and mitochondrial trees, we applied the method proposed by Kubatko [40]. The method assumes that incomplete lineage sorting (ILS) explains observed gene tree incongruence to some extent, and seeks to determine whether all variation in observed gene trees can be explained by ILS alone, as modeled by the coalescent process, or whether hybridization helps to explain significantly more the observed variation. Then, the Akaike information criterions (AIC) in each model (may or may not include hybridization scenarios) were compared to determine the best-fit model. For our data, two possible hybridization events were hypothesized. The first involved Trachypithecus, with parental taxa Semnopithecus and Presbytis, while the second involved the clade containing Piliocolobus and Procolobus, Colobus and the ancestor of Asian colobines.

By comparing the results from models with or without the hybridization events, the best-fit model (AIC = 3021.79, Figure 2F) was a tree in which Trachypithecus is the result of hybridization between Presbytis and Semnopithecus. The second best-fit model (AIC $=3023.57$, Figure 2I) comprised the tree that includes both tested hybridization events. AIC values for all seven other models were considerably higher (3072.25 - 4051.14). Since AIC values for the scenarios presented in Figures $2 \mathrm{~F}$ and $2 \mathrm{I}$ were the lowest and were within 2 of one another, both were considered plausible explanations for the observed gene tree discordances [41]. It is worth pointing out that the model used here to compute the AIC assumes that ILS is a possible source of gene trees incongruence. Since the two best-fit models include at least one hybridization event, it is clear that ILS alone 


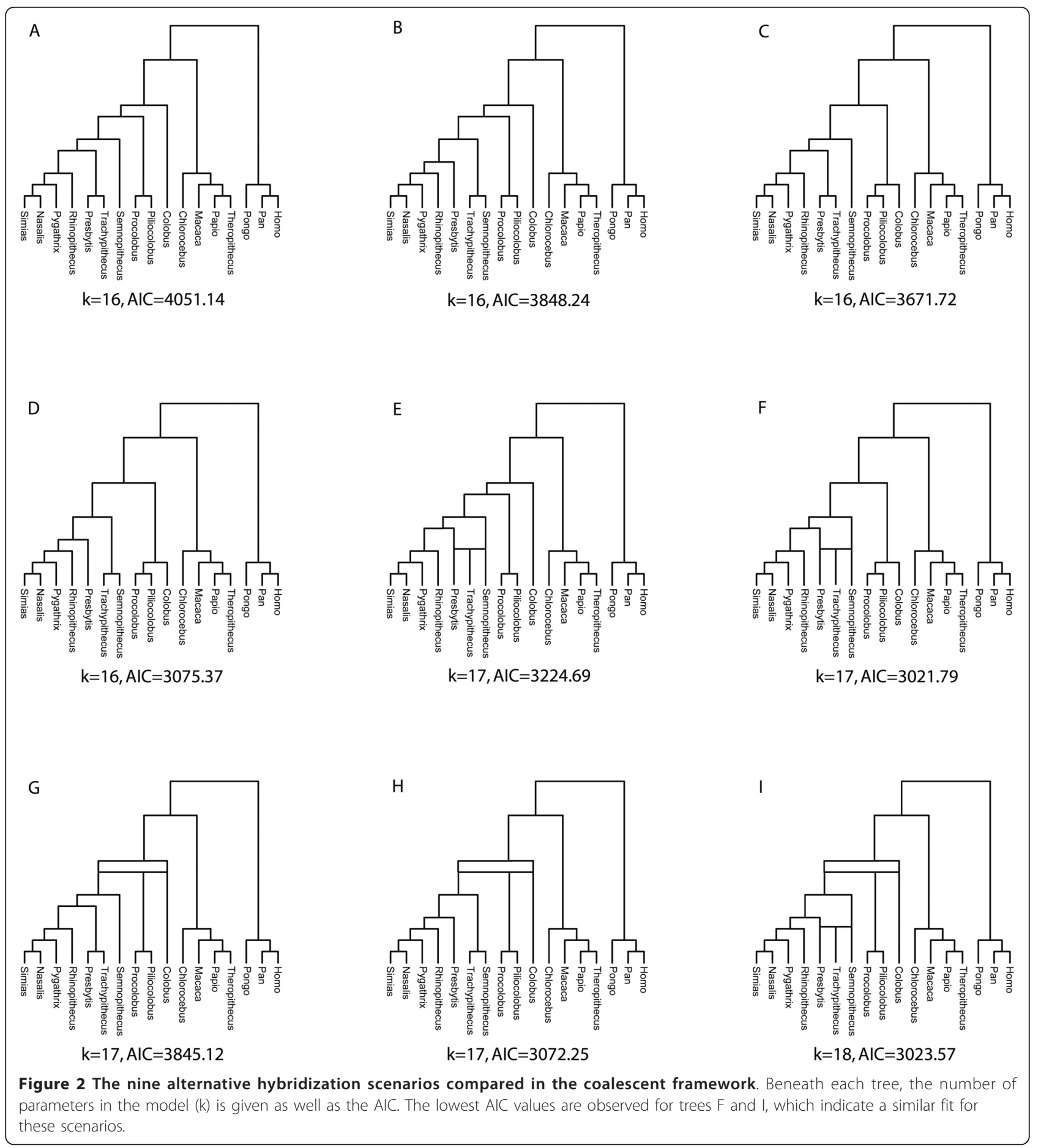

does not adequately describe the extent of incongruence in the observed gene trees.

\section{Discussion}

By combining presence/absence analysis of mobile elements with autosomal, X chromosomal, Y chromosomal and mitochondrial sequence data, the present study provides comprehensive insights into the evolutionary history of colobines. Most relationships are resolved and strongly supported by mobile elements and sequence data. Moreover, relationships and estimated divergence ages as obtained from different datasets are mainly congruent and in agreement with earlier studies [8-10,23,42-45]. Our study, however, also reveals significant discrepancies among gene trees. First, mitochondrial and nuclear sequence data suggest a monophyletic African colobine 
clade, while mobile elements provide evidence for a closer connection of the Piliocolobus/Procolobus clade to Asian genera than to Colobus. Second, mobile elements indicate close relationships between Semnopithecus and Trachypithecus, and between Presbytis and the odd-nosed monkeys. Nuclear sequence data support the former clade, but suggest Presbytis as basal among Asian colobines. In contrast, in the mitochondrial phylogeny, Presbytis and Trachypithecus are displayed as sister lineages, while the position of Semnopithecus remains ambiguous.

\section{Possible explanations for gene tree discordance}

Inadequate data, homoplasy, nucleotide composition, ILS or hybridization could be potential explanations for the observed differences [13-21]. For the mitochondrial dataset, at least for the African and Presbytis/Trachypithecus clades, incorrect branching patterns due to inadequate data or homoplasy are unlikely, since sufficient phylogenetic resolution with long internal branches is obtained. Likewise, a shift in nucleotide composition and differential sorting of ancestral mitochondrial lineages is implausible. Since the major shift in nucleotide composition was detected between colobines and non-colobines, it cannot be responsible for gene tree discordances among colobines. If the African and Presbytis/Trachypithecus clades are indeed the result of incomplete sorting of mitochondrial lineages, the mitochondrial divergence between respective genera should predate the nuclear splitting times, which is not the case (African colobines: 10.93 mya nuclear vs. 8.47 mya mitochondrial; Presbytis - Trachypithecus: 8.12 mya nuclear vs. 7.45 mya mitochondrial). However, the unresolved position of Semnopithecus among Asian colobines might have been affected by one or several of the above mentioned factors, or alternatively, might be the result of a true radiation-like divergence of lineages. For nuclear data, these factors are unlikely explanations as well for the branching of Trachypithecus and Semnopithecus, because ten independent insertions and sequence data from 12 nuclear loci clearly confirm their close relationship. More challenging are explanations for the discordant positions of Presbytis and the African genera among colobines in phylogenies revealed by mobile elements and nuclear sequence data. Mixed genomes due to differentially selected genes cannot be excluded, but interestingly, both mobile elements and nuclear sequence data (as revealed from single locus analysis) show no conflicting phylogenies themselves. Most prominent, however, the mobile element-based phylogeny is not rejected by nuclear sequence data, indicating that insufficient informative sites, as also suggested by the low resolution of phylogenetic relationships in single-locus analysis, in the latter dataset might display incorrect relationships. For the integration of mobile elements, homoplasy is typically regarded as minimal $[25,28,30]$, but ILS has been reported [36,39]. Only two and three integrations support the branching of Presbytis with odd-nosed monkeys and the paraphyly of African colobines, and alternative relationships cannot be rejected statistically. However, no inconsistent elements were detected and subtractive hybridizations specifically set up to screen for African colobine and Trachypithecus/Presbytis monophyly markers revealed no equivalent insertions. Accordingly, ILS seems to be an unreasonable explanation for our findings. Since the mobile element-based phylogeny is not rejected by nuclear sequence data and due to their reliability as molecular-cladistic markers, the phylogeny suggested by mobile elements is assumed to reflect the true nuclear phylogeny of colobines, although we explicitly note that mosaic genomes cannot be excluded.

Because all above-mentioned factors provide no sufficient explanation for the herein detected discordances between mitochondrial and nuclear phylogenies, we favor ancestral hybridization as the main reason for the discordant pattern. Furthermore, comparisons of models with and without hybridization in a model selection framework strongly support hybridization in the presence of ILS over models of ILS alone. In other words, even after ILS was taken into account as a factor in the observed incongruence among gene trees, we still found support for hybridization in the evolutionary history of these taxa. This refers at least to Asian colobines, but hybridization among African colobines cannot be excluded either by the method we applied here.

\section{Hybridization hypothesis}

Although bidirectional hybridization, which would be indicated by mixed genomes, cannot be excluded with our data, a female introgression event is hypothesized for African colobines. The direction of gene flow remains obscure due to the rapid diversification of the colobine ancestor in Africa, but female introgression from Piliocolobus/Procolobus into Colobus is indicated and gains further support by some biological data $[1,2]$. In contrast to Colobus, females in Piliocolobus and Procolobus tend to leave their natal groups, which was most likely also the case in their ancestor [1], and Colobus males are on average larger than Piliocolobus and Procolobus males [1], thus increasing the chance of hybridization between Colobus males and Piliocolobus/Procolobus females. Moreover, hybridization between both ancestral lineages is in principal possible, because (at least nowadays) they occur in sympatry over wide ranges of their distribution [1,2]. Accordingly, after the successive separation of Colobus and Piliocolobus/Procolobus from the Asian colobine ancestor, Piliocolobus/Procolobus females might have entered Colobus populations and 
hybridized with their males. Backcrossing of hybrid females with resident Colobus males might has led to the fixation of the Piliocolobus/Procolobus mitochondrial lineage in the hybrid population, while the original nuclear genome of Colobus increased again in every generation.

For Asian langurs, we propose male introgression from Semnopithecus into Trachypithecus followed by nuclear swamping. Both genera are similar in their morphology and general appearance $[2,46,47]$, but males in Semnopithecus are larger than in Trachypithecus [1]. Moreover, hybridization events due to (at least nowadays) partially overlapping ranges are generally possible $[1,2]$. Accordingly, after an initial separation, Semnopithecus males, which leave their natal group like most other primate males $[1,48]$, might have invaded Trachypithecus populations and hybridized successfully with the resident females. By backcrossing with further invading Semnopithecus males over a longer period, the Trachypithecus population might have accumulated nuclear material of Semnopithecus (nuclear swamping), while the mitochondrial genome remained Trachypithecuslike.

\section{Biogeographic implications}

By combining the available information, we develop the following extended dispersal scenario for colobines (Figure 3). The origin of the subfamily is most likely in Africa, which is in agreement with earlier suggestions $[1,49]$. On the African continent, Colobus split off first from the main stem $\sim 10.93$ mya, followed shortly afterwards by the progenitor of Piliocolobus and Procolobus. After this initial separation, hybridization between both lineages might have lasted until finally both mitochondrial lineages diverged ( 8.47 mya). Presumably, respective splitting and hybridization events took place in western Africa, because all three genera occur there in sympatry [1,2], and the most ancient splits among Piliocolobus and Colobus species are also found there [45]. The Asian colobine ancestor most likely invaded Eurasia via an emerging land bridge connecting Africa and the Arabian Peninsula in the late Miocene $[49,50]$. Whether a route into eastern Asia north or south of the Himalayas was chosen is a matter of speculation, but north of the Himalayas, on the Tibetan plateau, colobine fossils from the late Miocene were found, which is not the case south of the Himalayas [1]. Although not confirmed, the Hengduan Mountains in the border region of today's Burma, India

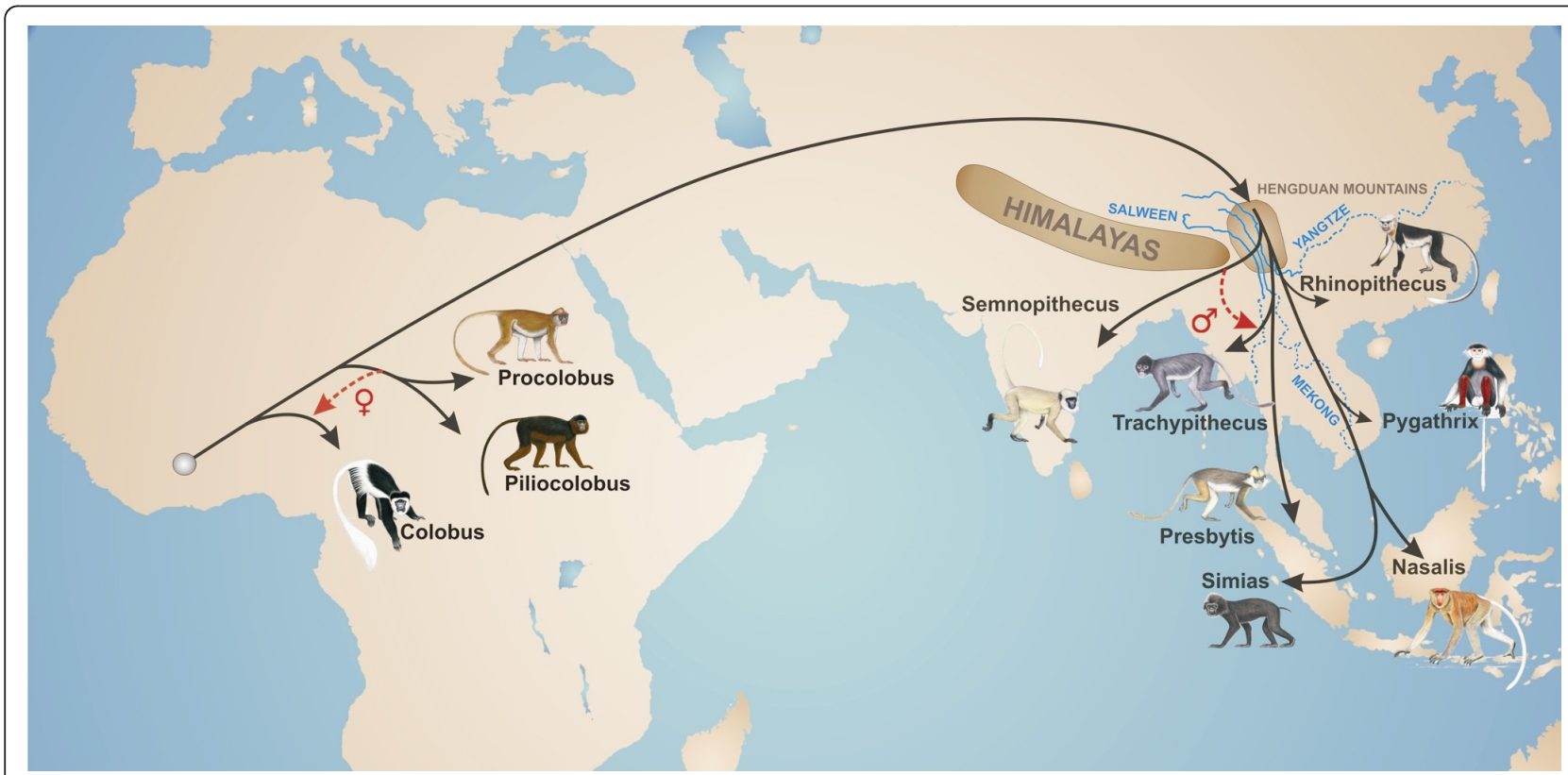

Figure 3 Dispersal scenario for colobine monkeys. Colobines most likely originated in western Africa. After the successive split of Colobus ( 10.9 mya) and a progenitor of Piliocolobus/Procolobus ( 10.7 mya) from the ancestor of Asian colobines, gene flow between both African lineages via female introgression from the Piliocolobus/Procolobus progenitor into Colobus occurred until 8.5 mya (displayed by red-dashed arrow). During the late Miocene, colobines invaded eastern Asia most likely via a route north of the Himalayas. After their arrival at the Hengduan Mountains, Asian colobines diversified into a lineage comprising a progenitor of the odd-nosed monkeys and Trachypithecus/Presbytis, and of Semnopithecus, which later colonized the Indian subcontinent. Shortly afterwards, Trachypithecus/Presbytis split off from odd-nosed monkeys, and migrated to southern mainland Asia, before finally both genera diverged from each other. In the region of today's Burma, Bangladesh and India, Semnopithecus and Trachypithecus came into secondary contact and hybridized until 2.6 mya (displayed by red-dashed arrow). In the latest Miocene, odd-nosed monkeys migrated from China to the south and expanded their range into Indochina and Sundaland. Nasalis and Simias finally separated from each other 1.1-1.9 mya. 
and China might have been a possible diversification hotspot $[4,51,52]$. In the region, all the larger Southeast Asian rivers (Mekong, Salween, Yangtze) rise, which are all wellknown as barriers for arboreal primates [53] and are all known to exist since at least the early Miocene [54]. Semnopithecus might have diverged as first lineage and invaded the Indian subcontinent. Subsequently, the progenitor of Presbytis and Trachypithecus separated from the odd-nosed monkey ancestor and migrated into southern mainland Asia. Afterwards, Presbytis diverged from Trachypithecus and entered first the Malaysian peninsular and later on Sundaland during periods of lowered sea levels [55]. Trachypithecus and Semnopithecus came into secondary contact and might have hybridized until the earliest Pleistocene. A potential contact zone could be the region of today's Bangladesh, Burma and the northeast of India, which is suggested as hybridization area for several primate species $[9,44,56]$. On the Asian mainland, odd-nosed monkeys successively migrated from China to the south and expanded their range into Indochina and Sundaland in the latest Miocene. The migration into Sundaland was probably via land bridges connecting the mainland with Sundaland islands during periods of lowered sea levels [55]. Finally, Nasalis on Borneo and Simias on the Mentawai islands west of Sumatra diverged in the Pleistocene. Due to the dating discrepancy (mitochondrial data: 1.88 mya, nuclear data: 1.06 mya), further gene flow between both genera after the initial separation cannot be excluded, especially considering that migration was repeatedly possible via land bridge connections during the Pleistocene [55].

\section{Conclusion}

Our study gives new and most comprehensive insights into the evolutionary history of colobine monkeys, and suggests hybridization among ancestral lineages as the most likely cause for the observed phylogenetic incongruences. Only the combination of maternally, paternally and bi-parentally inherited markers as well as the combination of sequence data with presence/absence patterns of mobile elements proved to be an adequate and reliable phylogenetic approach, particularly in revealing hybridization events. However, data from additional nuclear loci and a broader taxonomic sampling is required to fully understand hybridization mechanisms in colobines.

Hybridization among taxa is traditionally recognized as a factor leading to limited diversification, reproductive isolation and lowered fitness $[57,58]$, whereas our and earlier studies clearly indicate that hybridization played a prominent role in diversification and speciation of primates (for review see $[59,60]$ ). Hybridization events are genetically confirmed within all major primate lineages, mainly among species (e.g., [56,61-65]) but also between genera (e.g., $[9,44,66])$. Even for the human lineage, hybridization has been suggested as an important evolutionary mechanism [67-69].

Since male dispersal and female philopatry predominates in primates [48], male introgression, and if intensive backcrossing of hybrids with more invading males occurs, followed by nuclear swamping would be the most likely hybridization scenario. In fact, the hybridization among Asian langur genera is most likely the result of such an event. However, as proposed for African colobines, alternative mechanisms (e.g. female introgression) could also occur, promoted by a respective social organization, where female migration predominates.

\section{Methods}

\section{Sample collection and DNA extraction}

Blood, tissue or fecal samples from representatives of all ten colobine genera (Colobus, Piliocolobus, Procolobus, Presbytis, Trachypithecus, Semnopithecus, Rhinopithecus, Pygathrix, Nasalis, Simias) and several non-colobine taxa (Macaca, Papio, Theropithecus, Chlorocebus, Pongo, $\mathrm{Pan}$ ) were obtained from specimens kept in zoos or breeding facilities, or collected in the field (Table 2). Sample collection was conducted according to relevant German and international guidelines, including countries where we collected samples. Fecal samples were collected in a non-invasive way without disturbing, threatening or harming the animals. Blood samples were taken by veterinarians for diagnostic reasons to check

\section{Table 2 Origin and sample type of studied species}

\begin{tabular}{|c|c|c|}
\hline species & origin & $\begin{array}{l}\text { sample } \\
\text { type }\end{array}$ \\
\hline Colobus guereza & Cologne zoo, Germany & tissue \\
\hline Piliocolobus badius & Taï National Park, Ivory Coast & tissue \\
\hline Procolobus verus & Taï National Park, Ivory Coast & tissue \\
\hline $\begin{array}{l}\text { Semnopithecus } \\
\text { entellus }\end{array}$ & Dresden zoo, Germany & blood \\
\hline $\begin{array}{l}\text { Trachypithecus } \\
\text { obscurus }\end{array}$ & Wuppertal zoo, Germany & blood \\
\hline Presbytis melalophos & $\begin{array}{l}\text { Howletts Wild Animal Park, Great } \\
\text { Britain }\end{array}$ & tissue \\
\hline Pygathrix nemaeus & Cologne zoo, Germany & tissue \\
\hline $\begin{array}{l}\text { Rhinopithecus } \\
\text { avunculus }\end{array}$ & $\begin{array}{l}\text { Endangered Primate Rescue Center, } \\
\text { Vietnam }\end{array}$ & tissue \\
\hline Nasalis larvatus & Wilhelma Stuttgart, Germany & blood \\
\hline Simias concolor & $\begin{array}{l}\text { Siberut Conservation Programme, } \\
\text { Indonesia }\end{array}$ & feces \\
\hline Macaca sylvanus & Nuremberg zoo, Germany & blood \\
\hline Papio hamadryas & Munich zoo, Germany & blood \\
\hline $\begin{array}{l}\text { Theropithecus } \\
\text { gelada }\end{array}$ & Duisburg zoo, Germany & blood \\
\hline $\begin{array}{l}\text { Chlorocebus } \\
\text { aethiops }\end{array}$ & Paul-Ehrlich-Institute, Germany & blood \\
\hline Pongo abelii & Nuremberg zoo, Germany & blood \\
\hline Pan troglodytes & Munich zoo, Germany & blood \\
\hline
\end{tabular}


the health status of the respective individuals, and tissue samples were obtained only from deceased specimens. Total genomic DNA was extracted with the DNeasy Blood \& Tissue or QIAamp DNA Stool Mini kits from Qiagen following standard procedures.

\section{Analysis of mobile elements}

Due to their high copy number ( one million) and relatively small size ( $200 \mathrm{bp})$, the primate specific Alu elements were selected as molecular-cladistic markers. The presence or absence of mobile elements in different colobines at specific loci was tested via PCR using primers occupying the flanking region of the insertion site. Details on analyzed loci, primers and presence/absence pattern of mobile elements in studied species are listed in Additional file 1. For most loci, sequencing was neglected, but in relevant cases the insertion orthology was confirmed by sequencing, and direct repeats flanking the insertion as well as the original target site prior to transposition were traced.

In our study, we included published markers [9,23,35], which were further examined in previously untested genera, and newly detected integration loci (Additional file 1). Therefore, we performed subtractive hybridizations following described methods [9]. To avoid biased hybridization results, various species combinations were used as tracer and driver (hybridization 1: tracer Nasalis/Pygathrix, driver Presbytis; hybridization 2: tracer Nasalis/Pygathrix, driver Semnopithecus; hybridization 3: tracer Trachypithecus/Presbytis, driver Pygathrix; hybridization 4: tracer Presbytis, driver Semnopithecus; hybridization 5: tracer Piliocolobus/Colobus, driver Pygathrix). Besides Alu insertions, a LINE present in Piliocolobus and Procolobus in the studied Xq13.3 fragment was additionally applied as marker (Additional file 1).

Phylogenetic reconstructions using the MP algorithm were conducted in PAUP v4.0b10 [70]. Presence of an integration was coded as 1 , its absence as 0 , and missing data as '?'. Internal node support was obtained via a heuristic search with 10,000 bootstrap replications. To evaluate the reliability of the depicted relationships among colobines, various alternative tree topologies (Additional file 2) were assessed with the Kishino-Hasegawa test [71] with full optimization and 1,000 bootstrap replications in PAUP.

\section{Amplification and sequencing of nuclear loci}

Inter-exonic intron and exonic sequences were generated for six single-copy genes of the $\mathrm{Y}$ chromosome, five autosomal loci, and a fragment of the $\mathrm{X}$ chromosomal Xq13.3 region. With exception of the $S R Y$ gene (sexreversal, Y chromosome), all other Y chromosomal loci (DBY5: Dead Box, intron 5; SMCY7: SMC mouse homologue, intron 7; SMCY11: SMC mouse homologue, intron 11; UTY18: ubiquitous TPR motif, intron 18; ZFYLI: Zinc finger, last intron) have homologues on the $\mathrm{X}$ chromosome (X degenerate). As autosomal loci, we selected intron 11 of the von Willebrand Factor ( $v$ WF11), located on human chromosome 12, intron 3 of the serum albumin gene $(A L B 3$, human chromosome 4 ), intron 3 of the interstitial retinol-binding protein (IRBP3, human chromosome 10), intron 1 of the transition protein 2 (TNP2, human chromosome 16) and intron 1 of the transthyretin gene (TTR1, human chromosome 18). SRY, DBY5, SMCY7, SMCY11, UTY18, $\nu W F 11$ and a $\sim 4,300 \mathrm{bp}$ fragment of the Xq13.3 region were amplified using primers and PCR conditions as described [10,72-75] (Additional file 9). For the amplification of ZFYLI, ALB3, IRBP3, TNP2 and TTR1, new primers (Additional file 9) were designed on the basis of available primate sequences in GenBank. PCR conditions for the latter comprised a pre-denaturation step at $94^{\circ} \mathrm{C}$ for $2 \mathrm{~min}$, followed by 40 cycles each with denaturation at $94^{\circ} \mathrm{C}$ for $1 \mathrm{~min}$, annealing at varying temperatures (Additional file 9) for $1 \mathrm{~min}$, and extension at $72^{\circ} \mathrm{C}$ for $2 \mathrm{~min}$. At the end, a final extension step at $72^{\circ}$ $\mathrm{C}$ for 5 min was added. The results of all PCR amplifications were checked on $1 \%$ agarose gels. PCR products were cleaned with the Qiagen PCR Purification kit and subsequently sequenced on an ABI $3130 \times 1$ sequencer using the BigDye Terminator Cycle Sequencing kit. Alignments and sequences are available in TreeBASE (http://purl.org/phylo/treebase/phylows/study/TB2: S11179) and GenBank, respectively (for GenBank accession numbers see Additional file 10).

\section{Amplification and sequencing of mitochondrial genomes}

To reduce the likelihood of amplifying nuclear pseudogenes (numts), complete mitochondrial genomes from four colobine genera (Rhinopithecus, Pygathrix, Nasalis, Procolobus) were generated following an approach in which two overlapping $\sim 10,000 \mathrm{bp}$ long fragments were amplified via long-range PCR $[8,43]$. Due to degradation of DNA extracted from faeces, the mitochondrial genome of Simias was amplified via five overlapping fragments, each with a size of $\sim 5,000 \mathrm{bp}$. All long-range PCRs were performed with the SuperTaq Plus polymerase from Ambion following protocols of the supplier and primers as described $[8,43]$. Long-range PCR amplicons were separated on $1 \%$ agarose gels, excised from the gel, purified with the Qiagen Gel Extraction kit and used as template for nested PCRs. PCR conditions for all nested PCR amplifications were identical and comprised a pre-denaturation step at $94^{\circ} \mathrm{C}$ for $2 \mathrm{~min}$, followed by 30 cycles each with denaturation at $94^{\circ} \mathrm{C}$ for 1 min, annealing at $60^{\circ} \mathrm{C}$ for $1 \mathrm{~min}$, and extension at $72^{\circ} \mathrm{C}$ for $1.5 \mathrm{~min}$. At the end, a final extension step at $72^{\circ} \mathrm{C}$ for 5 min was added. Nested PCR products (900-1,200 
bp in length) were cleaned with the Qiagen PCR Purification kit and sequenced on an ABI $3130 \times 1$ sequencer. Sequences were assembled with Geneious v4.6.1 [76]. No inconsistent positions in overlapping regions were detected and all protein-coding genes were correctly translated. Annotation of mitochondrial genomes was conducted with the online program DOGMA [77] and manually inspected. Alignment and sequences are available in TreeBASE (http://purl.org/phylo/treebase/phylows/study/TB2:S11179) and GenBank, respectively (for GenBank accession numbers see Additional file 10).

\section{Statistical analysis of sequence data}

For phylogenetic reconstructions, all datasets comprised 17 sequences including each one representative of the ten colobine genera (Colobus, Piliocolobus, Procolobus, Trachypithecus, Semnopithecus, Presbytis, Rhinopithecus, Pygathrix, Nasalis, Simias), four cercopithecine genera (Papio, Theropithecus, Macaca, Chlorocebus), and three hominoid genera (Homo, Pan, Pongo), which were used as outgroup taxa. To complete datasets, we partly implemented sequences from GenBank (Additional file 10). Alignments for individual loci were generated with MAFFT v6 [78] and corrected by eye. In all alignments, poorly aligned positions and indels were removed with Gblocks v0.91b [79] using default settings (Additional file 8). For the mitochondrial dataset, also the D-loop region was excluded (dataset mtDNA1) and a second alignment, generated in Mesquite v2.6 [80], included solely protein-coding genes (dataset mtDNA2). For all datasets, uncorrected pairwise differences were estimated in PAUP (Additional file 7). Nucleotide composition for all and only parsimony-informative positions for the combined nuclear and both mitochondrial alignments was also estimated in PAUP (Additional file 4). To test whether datasets can be combined, we performed partition homogeneity tests in PAUP with 10,000 replications.

Phylogenetic trees were constructed with MP and NJ algorithms as implemented in PAUP as well as with ML and Bayesian algorithms, using the programs GARLI v0.951 [81] and MrBayes v3.1.2 [82,83]. For MP analyses, all characters were treated as unordered and equally weighted throughout. A heuristic search was performed with the maximum number of trees set to 100. For NJ, ML and Bayesian reconstructions, the optimal nucleotide substitution models for each locus and concatenated datasets were chosen using AIC as implemented in MODELTEST v3.7 [84] (Additional file 8). Relative support of internal nodes was assessed by bootstrap analyses with 10,000 (MP, NJ) or 500 replications (ML). In GARLI, only the model specification settings were adjusted according to the respective concatenated dataset, while all other settings were left at their default value. ML majority-rule consensus trees were calculated in PAUP. For Bayesian reconstructions, the datasets were partitioned treating each locus separately and each with its own substitution model. The solely protein-coding alignment of the mitochondrial genome (mtDNA2) was partitioned into codon positions. We used four independent Markov Chain Monte Carlo (MCMC) runs with the default temperature of 0.1 . Four repetitions were run for 10,000,000 generations with tree and parameter sampling occurring every 100 generations. The first $25 \%$ of samples were discarded as burnin, leaving 75,001 trees per run. PPs for each split and a phylogram with mean branch lengths were calculated from the posterior density of trees.

To evaluate the reliability of obtained relationships among colobines, various alternative tree topologies (Additional file 2) were tested with the ShimodairaHasegawa test [85] with full optimization and 1,000 bootstrap replications in PAUP.

\section{Divergence age estimation}

A Bayesian MCMC method, which employs a relaxed molecular clock approach [86], as implemented in BEAST v1.4.8 [87], was used to estimate divergence times. Therefore, a relaxed lognormal model of lineage variation and a Yule prior for branching rates was assumed. Divergence times were calculated for each locus separately and for the combined nuclear dataset. The latter was partitioned treating each locus as distinct unit. The mitochondrial alignment comprising solely protein-coding genes (mtDNA2) was partitioned into codon positions and the substitution model, rate heterogeneity and base frequencies were unlinked across codon positions. Optimal nucleotide substitution models were chosen using AIC in MODELTEST.

As calibrations we used the fossil-based divergence between Homo and Pan, which has been dated at 6-7 mya [88-90], the separation of Pongo from the Homol Pan lineage $\sim 14$ mya [91], the split between Theropithecus and Papio 4 mya [92,93], and the divergence of hominoids and cercopithecoids $\sim 24$ mya [94-96]. Instead of hardbounded calibration points, we used the published dates as a normal distribution prior for the respective node. For the Homo - Pan divergence, this translates into a normal distribution with a mean of 6.5 mya and a standard deviation (SD) of 0.5 mya, for the separation of Pongo from the Homo/Pan clade into a mean of 14.0 mya and a SD of 1.0 mya, for the Theropithecus - Papio split into a mean of 4.0 mya and a SD of 0.5 mya, and for the hominoid - cercopithecoid divergence into a mean of 24 mya and a SD of 2 mya.

Since the estimation of phylogenetic relationships was not the main aim of this analysis, we used an a-priori fixed tree topology as obtained from mobile elements 
(Figure 1A) for the calculation from nuclear sequence data. Four replicates were run for 10,000,000 generations with tree and parameter sampling occurring every 100 generations. The adequacy of a $10 \%$ burnin and convergence of all parameters were assessed by visual inspection of the trace of the parameters across generations using TRACER v1.4.1 [97]. Subsequently, the sampling distributions were combined (25\% burnin) using the software LogCombiner v1.4.8 and a consensus chronogram with node height distribution was generated and visualized with TreeAnnotator v1.4.8 and FigTree v1.2.2 [98].

\section{Inferring hybridization in the presence of incomplete lineage sorting}

Statistical support for putative hybridization scenarios was assessed with the method proposed by Kubatko [40], in which statistical model selection techniques (e. g., AIC) are used to compare species trees that may or may not include hybridization scenarios. For our data, we hypothesized two possible hybridization events (for details see Results). The estimated gene trees used as input were those derived from single locus tree reconstructions (Additional file 3) and branch lengths as estimated in BEAST. To estimate evolutionary rates for individual loci, we followed the suggestion of Yang [99] (see also [100]) and computed for each gene the average pairwise sequence divergence of each ingroup (colobine) sequence to the outgroup (non-colobine) taxa. We then assigned to each locus a rate that was calculated by dividing the mean pairwise divergence for that locus by the median of the entire set of pairwise divergences (Additional file 8). To convert gene tree branch lengths to coalescent units, we considered two effective population sizes, 50,000 and 100,000, and used a generation time of 5 years. Since the results were identical in terms of the trees preferred, we show here the results only for effective population size 50,000. For haploid loci (mitochondrial genome, Y chromosomal loci), we additionally divided the rate by 2 (see [100]). We compared a total of nine species trees (four corresponding to no hybridization, four corresponding to single hybridization events, and one that included both hybridization scenarios, Figure 2). The AIC was computed for each tree using the STEM software [100]. Models with AIC values within 2 of one another were regarded as providing similar fit to the data [41].

\section{Additional material}

Additional file 1: Additional Table 1. Presence/absence pattern

location, primers and PCR product sizes of mobile elements

Additional file 2: Additional Table 2. Alternative tree topology tests
Additional file 3: Additional Figure 1. Single-locus phylogenetic trees (80\% majority rule)

Additional file 4: Additional Figure 2. Nucleotide composition of both mitochondrial and the combined nuclear datasets

Additional file 5: Additional Figure 3. Phylograms based on the mitochondrial and combined nuclear datasets

Additional file 6: Additional Table 3. Divergence ages in mya estimated for each locus separately

Additional file 7: Additional Table 4. Uncorrected pairwise nucleotide differences for each locus

Additional file 8: Additional Table 5. Locus-specific information including alignment length, number of variable sites, selected substitution model and estimated evolutionary rates

Additional file 9: Additional Table 6. Primers and PCR conditions for the amplification of nuclear loci

Additional file 10: Additional Table 7. GenBank accession numbers

\section{Acknowledgements}

We thank the zoos in Cologne, Dresden, Duisburg, Howletts, Munich, Nuremberg, Stuttgart and Wuppertal, and Roland Plesker and Werner Schempp for providing samples. We are also grateful to our colleagues at Bogor Agricultural University (IPB) and the Indonesian Institute for Science (LIPI) as well as the Ivorian authorities, the Ministry of the Environment and Forests, the Ministry of Research, the directorship of Taï National Park and the Swiss Research Centre for providing the necessary field research permits and documents. No international and national rules and regulations have been violated during sampling and shipping. We thank Vanessa Roden, Laura Zidek, Linn Groeneveld, Daniel Stahl and two anonymous reviewers for valuable comments on an earlier version of the manuscript and statistical help. This work was supported by the German Primate Center and the Biodiversitäts-Pakt of the Wissenschaftsgemeinschaft Gottfried-Wilhelm Leibniz to CR, DZ and LW, and by grants from the National Institutes of Health to MAB (RO1 GM59290) and JX (K99 HG005846).

\section{Author details}

${ }^{1}$ Primate Genetics Laboratory, German Primate Center, Kellnerweg 4, 37077 Göttingen, Germany. ${ }^{2}$ Gene Bank of Primates, German Primate Center, Kellnerweg 4, 37077 Göttingen, Germany. ${ }^{3}$ Cognitive Ethology Laboratory, German Primate Center, Kellnerweg 4, 37077 Göttingen, Germany. ${ }^{4}$ Departments of Statistics and Evolution, Ecology and Organismal Biology, The Ohio State University, Columbus, Ohio 43210, USA. ${ }^{5}$ Reproductive Biology Unit, German Primate Center, Kellnerweg 4, 37077 Göttingen, Germany. ${ }^{6}$ Department of Anatomical Sciences, State University of New York, Stony Brook, New York 11794-8081, USA. ${ }^{7}$ Department of Human Genetics, University of Utah, 15 North 2030 East, Salt Lake City, Utah 84112, USA. ${ }^{8}$ Department of Biological Sciences, Louisiana State University, 202 Life Sciences Building, Baton Rouge, Louisiana 70803, USA. ${ }^{9}$ Research Group Emerging Zoonoses, Robert Koch Institute, Postfach 650261, 13302 Berlin, Germany. ${ }^{10}$ Primate Research Center and Department of Biology, Bogor Agricultural University, Jl. Lodaya II/5, Bogor 16151, Indonesia. ${ }^{11}$ Frankfurt Zoological Society, Endangered Primate Rescue Center, Cuc Phuong National Park, Nho Quan District, Ninh Binh Province, Vietnam. ${ }^{2}$ Stem Cell Biology Unit, German Primate Center, Kellnerweg 4, 37077 Göttingen, Germany.

\section{Authors' contributions}

CR designed the study, collected samples, did laboratory work, analyzed data, and wrote the paper. DZ designed the study, analyzed data, and wrote the paper. LSK, JX, MAB and MB analyzed data and wrote the paper. CS, MY, DM did laboratory work. SDN and LW wrote the paper. FHL, TZ, DPF and TN provided valuable samples and wrote the paper. $\mathrm{MO}$ did laboratory work, analyzed data and wrote the paper. All authors read and approved the final manuscript.

Received: 18 October 2010 Accepted: 24 March 2011 Published: 24 March 2011 
References

1. Davies AG, Oates JF: Colobine Monkeys - Their Ecology, Behaviour and Evolution Cambridge: Cambridge University Press; 1994

2. Groves CP: Primate Taxonomy Washington DC: Smithsonian Institution Press; 2001

3. Groves CP: A Theory of Human and Primate Evolution Oxford: Clarendon Press; 1989.

4. Jablonski NG: Natural History of the Doucs and Snub-Nosed Monkeys New Jersey: World Scientific Publishing Company; 1998.

5. Jablonski NG: Primate evolution - in and out of Africa. Curr Biol 1999, 9: R119-R122.

6. Stewart CB, Disotell TR: Primate evolution - in and out of Africa. Curr Bio 1999, 9:R120-R122.

7. Zhang YP, Ryder OA: Primate evolution - in and out of Africa. Curr Biol 1999, 9:R119-R120

8. Sterner KN, Raaum RL, Zhang YP, Stewart CB, Disotell TR: Mitochondrial data support an odd-nosed colobine clade. Mol Phylogenet Evol 2006, 40:1-7.

9. Osterholz $M$, Walter $L$, Roos $C$ : Phylogenetic position of the langur genera Semnopithecus and Trachypithecus among Asian colobines, and genus affiliations of their species groups. BMC Evol Biol 2008, 8:58

10. Ting N, Tosi AJ, Li Y, Zhang YP, Disotell TR: Phylogenetic incongruence between nuclear and mitochondrial markers in the Asian colobines and the evolution of the langurs and leaf monkeys. Mol Phylogenet Evol 2008, 46:466-474.

11. Napier JR, Napier PH: A Handbook of Living Primates London: Academic Press; 1967.

12. Szalay FS, Delson E: Evolutionary History of the Primates New York: Academic Press; 1979.

13. Barton NH: The role of hybridization on evolution. Mol Ecol 2001, 10:551-568.

14. Nichols R: Gene trees and species trees are not the same. Trends Ecol Evol 2001, 16:358-364

15. Funk D, Omland K: Species-level paraphyly and polyphyly: frequency, causes, and consequences, with insights from animal mitochondrial DNA. Ann Rev Ecol Evol Syst 2003, 34:397-423.

16. Avise JC: Molecular Markers, Natural History, and Evolution Sunderland, MA: Sinauer Associates; 2004

17. Seehausen O: Hybridization and adaptive radiation. Trends Ecol Evol 2004, 19:198-207.

18. McCracken $\mathrm{K}$, Sorenson M: Is homoplasy or lineage sorting the source of incongruent mtDNA and nuclear gene trees in the stiff-tailed ducks (Nomonyx-Oxyyura)? Syst Biol 2005, 54:35-55.

19. Pollard DA, lyer VN, Moses AM, Eisen MB: Widespread discordance of gene trees with species tree in Drosophila: evidence for incomplete lineage sorting. PLOS Genet 2006, 2:e173.

20. Koblmüller S, Duftner N, Sefc KM, Aibara M, Stipacek M, Blanc M, Egger B, Sturmbauer C: Reticulate phylogeny of gastropod-shell-breeding cichlids from Lake Tanganyika - the result of repeated introgressive hybridization. BMC Evol Biol 2007, 7:7

21. Philippe $H$, Laurent J: How good are deep phylogenetic trees? Curr Opin Genetics Dev 1998, 8:616-623.

22. Petit RJ, Excoffier L: Gene flow and species delimitation. Trends Ecol Evol 2009, 24:386-393.

23. Xing J, Wang H, Han K, Ray DA, Huang CH, Chemnick LG, Stewart CB, Disotell TR, Ryder OA, Batzer MA: A mobile element based phylogeny of Old World monkeys. Mol Phylogenet Evol 2005, 37:872-880.

24. Batzer MA, Deininger PL: A human-specific subfamily of Alu sequences. Genomics 1991, 9:481-487.

25. Okada N: SINEs. Curr Opin Genet Dev 1991, 1:498-504.

26. Shedlock AM, Okada N: SINE insertions: powerful tools for molecular systematics. Bioessays 2000, 22:148-160.

27. Salem AH, Ray DA, Batzer MA: Identity by descent and DNA sequence variation of human SINE and LINE elements. Cytogenet Genome Res 2005, 108:63-72.

28. Schmitz J, Roos C, Zischler H: Primate phylogeny: molecular evidence from retroposons. Cytogenet Genome Res 2005, 108:26-37.

29. Van de Lagemaat LN, Gagnier L, Medstrand P, Mager DL: Genomic deletions and precise removal of transposable elements mediated by short identical DNA segments in primates. Genome Res 2005, 15:1243-1249.
30. Ray DA, Xing J, Salem AH, Batzer MA: SINEs of a nearly perfect character Syst Biol 2006, 55:928-935.

31. Schmitz J, Ohme M, Zischler H: SINE insertions in cladistic analyses and the phylogenetic affiliations of Tarsius bancanus to other primates. Genetics 2001, 157:777-784.

32. Salem AH, Ray DA, Xing J, Callinan PA, Myers JS, Hedges DJ, Garber RK, Witherspoon DJ, Jorde LB, Batzer MA: Alu elements and hominid phylogenetics. Proc Natl Acad Sci USA 2003, 22:12787-12791.

33. Roos C, Schmitz J, Zischler H: Primate jumping genes elucidate strepsirrhine phylogeny. Proc Natl Acad Sci USA 2004, 101:10650-10654

34. Ray DA, Xing J, Hedges DJ, Hall MA, Laborde ME, Anders BA, White BR, Stoilova N, Fowlkes JD, Landry KE, Chemnick LG, Ryder OA, Batzer MA: Alu insertion loci and platyrrhine primate phylogeny. Mol Phylogenet Evol 2005, 35:117-126.

35. Herke SW, Xing J, Ray DA, Zimmermann JW, Cordaux R, Batzer MA: A SINEbased dichotomous key for primate identification. Gene 2007, 390:39-51.

36. Xing J, Wang H, Zhang Y, Ray DA, Tosi AJ, Disotell TR, Batzer MA: A mobile element based evolutionary history of guenons (Tribe Cercopithecini). BMC Biol 2007, 5:5

37. Xing J, Witherspoon DJ, Ray DA, Batzer MA, Jorde LB: Mobile DNA elements in primate and human evolution. Am J Phys Anthropol 2007 45(Suppl):2-19.

38. Osterholz M, Walter $\mathrm{L}$, Roos C: Retropositional events consolidate the branching order among New World monkey genera. Mol Phylogenet Evol 2009, 50:507-513.

39. Li J, Han K, Xing J, Kim HS, Rogers J, Ryder OA, Disotell T, Yue B, Batzer MA: Phylogeny of macaques (Cercopithecidae: Macaca) based on Alu elements. Gene 2009, 448:242-249.

40. Kubatko LS: Identifying hybridization events in the presence of coalescence via model selection. Syst Biol 2009, 58:478-488.

41. Burnham KP, Anderson DR: Model Selection and Multimodel Inference: A Practical Information-Theoretic Approach New York: Springer; 2002.

42. Goodman M, Porter CA, Czelusniak J, Page SL, Schneider H, Shoshani J, Gunnell G, Groves CP: Toward a phylogenetic classification of primates based on DNA evidence complemented by fossil evidence. Mol Phylogenet Evol 1998, 9:585-598.

43. Raaum RL, Sterner KN, Noviello CM, Stewart CB, Disotell TR: Catarrhine primate divergence dates estimated from complete mitochondrial genomes: concordance with fossil and nuclear DNA evidence. J Hum Evol 2005, 48:237-257.

44. Karanth KP, Singh L, Collura RV, Stewart CB: Molecular phylogeny and biogeography of langurs and leaf monkeys of South Asia (Primates: Colobinae). Mol Phylogenet Evol 2008, 46:683-694.

45. Ting N: Mitochondrial relationships and divergence dates of the African colobines: evidence of Miocene origins for the living colobus monkeys. $\lrcorner$ Hum Evol 2008, 55:312-325.

46. Brandon-Jones D: Colobus and leaf monkeys. In Encylopedia of Mammals. Edited by: MacDonald ID. London: George Allen and Unwin; 1984:398-408.

47. Strasser E, Delson E: Cladistic analysis of cercopithecid relationships. Hum Evol 1987, 16:81-99.

48. Pusey AE, Packer C: Dispersal and philopatry. In Primate Societies. Edited by: Smuts BB, Cheney DL, Seyfarth RM, Wrangham RW, Struhsaker TT. Chicago: University of Chicago Press; 1987:250-266

49. Stewart CB, Disotell TR: Primate evolution - in and out of Africa. Curr Biol 1998, 8:R582-R588

50. Whybrow PJ: Land movements and species dispersal. In The Cambridge Encyclopedia of Human Evolution. Edited by: Jones S, Martin RE, Pilbeam D. Cambridge: Cambridge University Press; 1992:169-173.

51. Peng $Y Z$, Pan RL, Jablonski N: Classification and evolution of Asian colobines. Folia Primatol 1993, 60:106-117

52. Thinh VN, Mootnick AR, Geissmann T, Li M, Ziegler T, Agil M, Moisson P Nadler $T$, Walter $L$, Roos $C$ : Mitochondrial evidence for multiple radiations in the evolutionary history of small apes. BMC Evol Biol 2010, 10:74.

53. Meijaard E, Groves CP: The geography of mammals and rivers in mainland Southeast Asia. In Primate Biogeography. Edited by: Lehman SM, Fleagle JG. New York: Springer; 2006:305-329.

54. Hallet B, Molnar P: Distorted drainage basins as markers of crustal strain east of the Himalayas. J Geophys Res 2001, 106:13697-13709.

55. Miller KG, Kominz MA, Browning JV, Wright JD, Mountain GS, Katz ME Sugarman PJ, Cramer BS, Christie-Blick N, Pekar SF: The phanerozoic record of global sea-level change. Science 2005, 310:1293-1298. 
56. Chakraborty D, Ramakrishnan U, Panor J, Mishra C, Sinha A: Phylogenetic relationships and morphometric affinities of the Arunachal macaque Macaca munzala, a newly described primate from Arunachal Pradesh, northeastern India. Mol Phylogenet Evol 2007, 44:838-849.

57. Darwin C: On the Origin of Species by Means of Natural Selection or the Preservation of Favoured Races in the Struggle of Life London: John Murray; 1859.

58. Mayr E: Animal Species and Evolution Cambridge, MA: Belknap Press; 1963.

59. Arnold ML, Meyer A: Natural hybridization in primates: one evolutionary mechanism. Zoology 2006, 109:261-276.

60. Zinner D, Arnold ML, Roos C: The strange blood: natural hybridization in primates. Evol Anthropol

61. Cortés-Ortiz L, Duda TF Jr, Canales-Espinosa D, Garcia-Orduna F, RodriguezLuna E, Bermingham E: Hybridization in large-bodied New World primates. Genetics 2007, 176:2421-2425.

62. Thalmann O, Fischer A, Lankester F, Pääbo S, Vigilant L: The complex evolutionary history of gorillas: insights from genomic data. Mol Biol Evol 2007, 24:146-158.

63. Rumpler Y, Warter S, Hauwy M, Fausser JL, Roos C, Zinner D: Comparing chromosomal and mitochondrial phylogenies of sportive lemurs (genus Lepilemur, Primates). Chromosome Res 2008, 16:1143-1158.

64. Merker S, Driller C, Perwitasari-Farajallah D, Pamungkas J, Zischler H: Elucidating geological and biological processes underlying the diversification of Sulawesi tarsiers. Proc Natl Acad Sci USA 2009, 106:8459-8464

65. Zinner D, Groeneveld LF, Keller C, Roos C: Mitochondrial phylogeography of baboons (Papio spp.) - indication for introgressive hybridization? BMC Evol Biol 2009, 9:83.

66. Zinner D, Arnold ML, Roos C: Is the new primate genus Rungwecebus a baboon? PLOS ONE 2009, 4:e4859.

67. Pääbo S: The mosaic that is our genome. Nature 2003, 421:409-412.

68. Patterson N, Richter DJ, Gnerre S, Lander ES, Reich D: Genetic evidence for complex speciation of humans and chimpanzees. Nature 2006, 441:1103-1108.

69. Green RE, Krause J, Briggs AW, Maricic T, Stenzel U, Kircher M, Patterson N Li H, Zhai W, Fritz MH, Hansen NF, Durand EY, Malaspinas AS, Jensen JD, Marques-Bonet T, Alkan C, Prüfer K, Meyer M, Burbano HA, Good JM, Schultz R, Aximu-Petri A, Butthof A, Höber B, Höffner B, Siegemund M, Weihmann A, Nusbaum C, Lander ES, Russ C, Novod N, Affourtit J, Egholm M, Verna C, Rudan P, Brajkovic D, Kucan Z, Gusic I, Doronichev VB, Golovanova LV, Lalueza-Fox C, de la Rasilla M, Fortea J, Rosas A Schmitz RW, Johnson PL, Eichler EE, Falush D, Birney E, Mullikin JC, Slatkin M, Nielsen R, Kelso J, Lachmann M, Reich D, Pääbo S: A draft sequence of the Neandertal genome. Science 2010, 328:710-722.

70. Swofford DL: PAUP* Phylogenetic Analysis using Parsimony (*and other Methods), Version 4 Sunderland, MA: Sinauer Associates; 2003.

71. Kishino $H$, Hasegawa M: Evaluation of the maximum likelihood estimate of the evolutionary tree topologies from DNA sequence data, and the branching order in Hominoidea. J Mol Evol 1989, 29:170-179.

72. Whitfield LS, Lovell-Badge R, Goodfellow PN: Rapid sequence evolution of the mammalian sex-determining gene SRY. Nature 1993, 364:713-715.

73. Chaves R, Sampaio I, Schneider MP, Schneider H, Page SL, Goodman M: The place of Callimico goeldii in the callithrichine phylogenetic tree: evidence from von Willebrand factor gene intron 11 sequences. Mol Phylogenet Evol 1999, 13:392-404.

74. Hellborg L, Ellegren $\mathrm{H}$ : $\mathrm{Y}$ chromsome conserved anchored tagged sequences (YCATS) for the analysis of mammalian male-specific DNA. Mol Ecol 2003, 12:283-291.

75. Tosi AJ, Detwiler KM, Disotell TR: X-chromosomal window into the evolutionary history of the guenons (Primates: Cercopithecini). Mol Phylogenet Evol 2005, 36:58-66.

76. Drummond AJ, Kearse M, Heled J, Moir R, Thierer T, Ashton B, Wilson A, Stones-Havas S: Geneious, version 4.6.1. 2009 [http://www.geneious.com]

77. Wyman SK, Jansen RK, Boore JL: Automatic annotation of organellar genomes with DOGMA. Bioinformatics 2004, 20:3252-3255.

78. Katoh K, Kuma K, Toh H, Miyata T: MAFFT version 5: improvement in accuracy of multiple sequence alignment. Nucleic Acids Res 2005, 33:511-518.

79. Castresana J: Selection of conserved blocks from multiple alignments for their use in phylogenetic analysis. Mol Biol Evol 2000, 17:540-552.
80. Maddison WP, Maddison DR: Mesquite: a modular system for evolutionary analysis, version 2.6. 2009 [http://mesquite.org].

81. Zwickl DJ: Genetic algorithm approaches for the phylogenetic analysis of large biological sequence data sets under the maximum likelihood criterion. PhD thesis Texas University, Austin; 2006.

82. Huelsenbeck JP, Ronquist F, Nielsen R, Bollback JP: Bayesian inference of phylogeny and its impact on evolutionary biology. Science 2001, 294:2310-2314.

83. Ronquist F, Huelsenbeck JP: MrBayes 3: Bayesian phylogenetic inference under mixed models. Bioinformatics 2003, 19:1572-1574.

84. Posada D, Crandall KA: Modeltest: testing the model of DNA substitution. Bioinformatics 1998, 14:817-818.

85. Shimodaira $H$, Hasegawa M: Multiple comparisons of log-likelihoods with applications to phylogenetic inference. Mol Biol Evol 1999, 16:1114-1116.

86. Drummond AJ, Ho SYW, Phillips MJ, Rambaut A: Relaxed phylogenetics and dating with confidence. PLoS Biol 2006, 4:e88.

87. Drummond AJ, Rambaut A: BEAST: Bayesian evolutionary analysis by sampling trees. BMC Evol Biol 2007, 7:214.

88. Vignaud P, Duringer P, Mackaye HT, Likius A, Blondel C, Boisserie JR, De Bonis L, Eisenmann V, Etienne ME, Geraads D, Guy F, Lehmann T, Lihoreau F, Lopez-Martinez N, Mourer-Chauviré C, Otero O, Rage JC, Schuster M, Viriot L, Zazzo A, Brunet M: Geology and palaeontology of the Upper Miocene Toros-Menalla hominid locality, Chad. Nature 2002, 418:152-155.

89. Brunet M, Guy F, Pilbeam D, Lieberman DE, Likius A, Mackaye HT, Ponce de León MS, Zollokofer CP, Vignaud P: New material of the earliest hominid from the Upper Miocene of Chad. Nature 2005, 434:752-755.

90. Lebatard $A E$, Bourlès $D L$, Duringer $P$, Jolivet $M$, Braucher $R$, Carcaillet J, Schuster M, Arnaud N, Monié P, Lihoreau F, Likius A, Mackaye HT, Vignaud P, Brunet M: Cosmogenic nuclide dating of Sahelanthropus tchadensis and Australopithecus bahrelghazali: Mio-Pliocene hominids from Chad. Proc Natl Acad Sci USA 2008, 105:3226-3231.

91. Kelley J: The hominoid radiation in Asia. In The Primate Fossil Record. Edited by: Hartwig WC. Cambridge: Cambridge University Press; 2002:369-384.

92. Leakey MG: Evolution of Theropithecus in the Turkana Basin. In Theropithecus, the Rise and Fall of a Primate Genus. Edited by: Jablonski NG. Cambridge: Cambridge University Press; 1993:85-124.

93. Delson E: Cercopithecinae. In Encyclopedia of Human Evolution and Prehistory. Edited by: Delson E, Tattersall I, Van Couvering JA, Brooks AS. New York: Garland Publishing Inc; 2000:166-171, 2000.

94. Benefit BR, McCrossin ML: The Victoriapithecidae, Cercopithecoidea. In The Primate Fossil Record. Edited by: Hartwig WC. Cambridge: Cambridge University Press; 2002:241-253

95. Young NM, MacLatchy L: The phylogenetic position of Moropithecus. $J$ Hum Evol 2004, 46:163-184

96. Zalmout IS, Sanders WJ, MacLatchy LM, Gunnell GF, Al-Mufarreh YA, Ali MA Nasser AAH, Al-Masari AM, Al-Sobhi SA, Nadhra AO, Matari AH, Wilson JA, Gingerich PD: New Oligocene primate from Saudi Arabia and the divergence of apes and Old World monkeys. Nature 2010, 466:360-365

97. Rambaut A, Drummond AJ: Tracer: MCMC trace analysis tool, version 1.4.1. Institute of Evolutionary Biology, University of Edinburgh; 2007 [http:// tree.bio.ed.ac.uk/software/tracer/].

98. Rambaut A: FigTree: Tree figure drawing tool, version 1.2.2. Institute of Evolutionary Biology, University of Edinburgh; 2008 [http://tree.bio.ed.ac.uk/ software/figtree/].

99. Yang Z: Likelihood and Bayes estimation of ancestral population sizes in hominoids using data from multiple loci. Genetics 2002, 162:1811-1823.

100. Kubatko LS, Carstens BC, Knowles LL: STEM: species tree estimation using maximum likelihood for gene trees under coalescence. Bioinformatics 2009, 25:971-973.

doi:10.1186/1471-2148-11-77

Cite this article as: Roos et al:: Nuclear versus mitochondrial DNA:

evidence for hybridization in colobine monkeys. BMC Evolutionary Biology 2011 11:77 

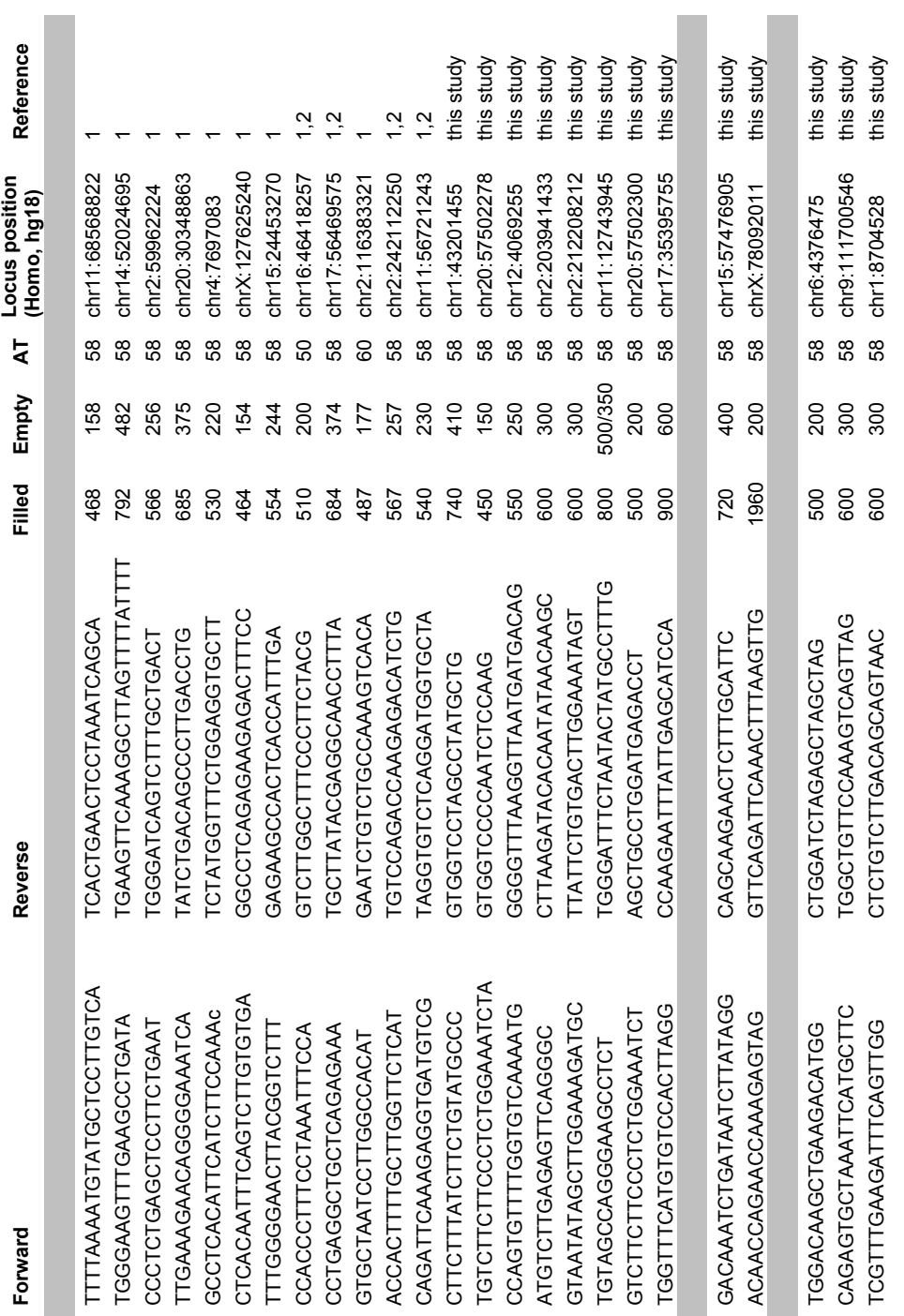


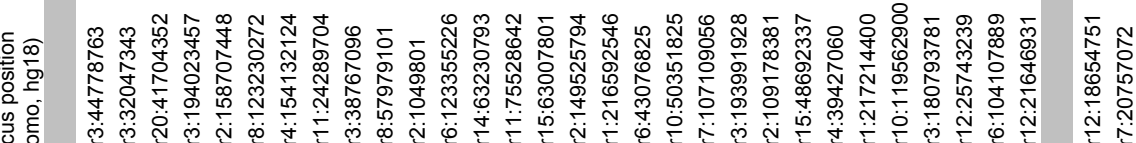

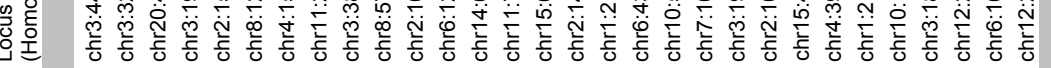

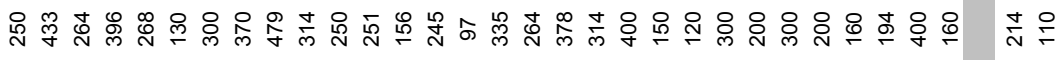

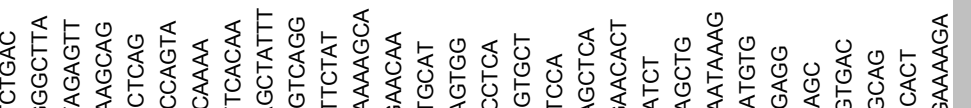

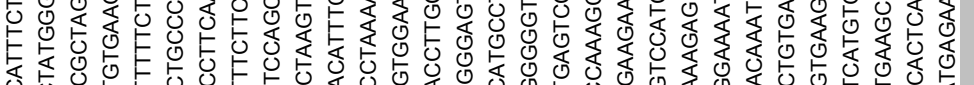

$\vec{z}$

z

ฐ

$\Sigma$

으

岁

ฉ

$000 \pm 000 \pm 0000000000000000000 \pm 00$

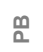

ن

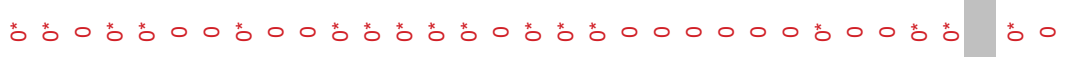

0

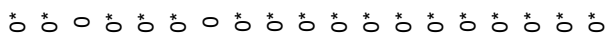

홍

$\sum$

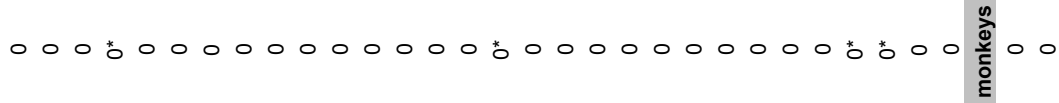

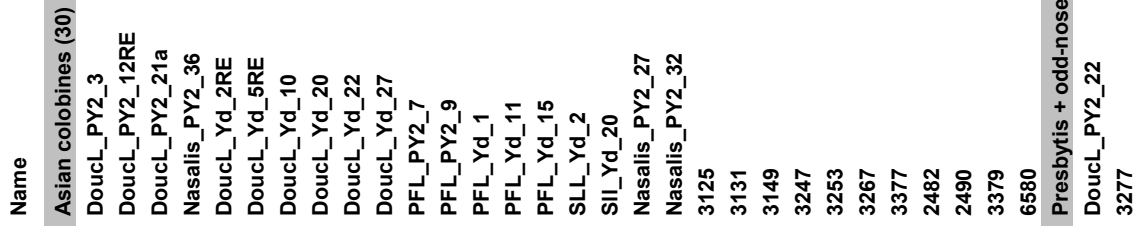




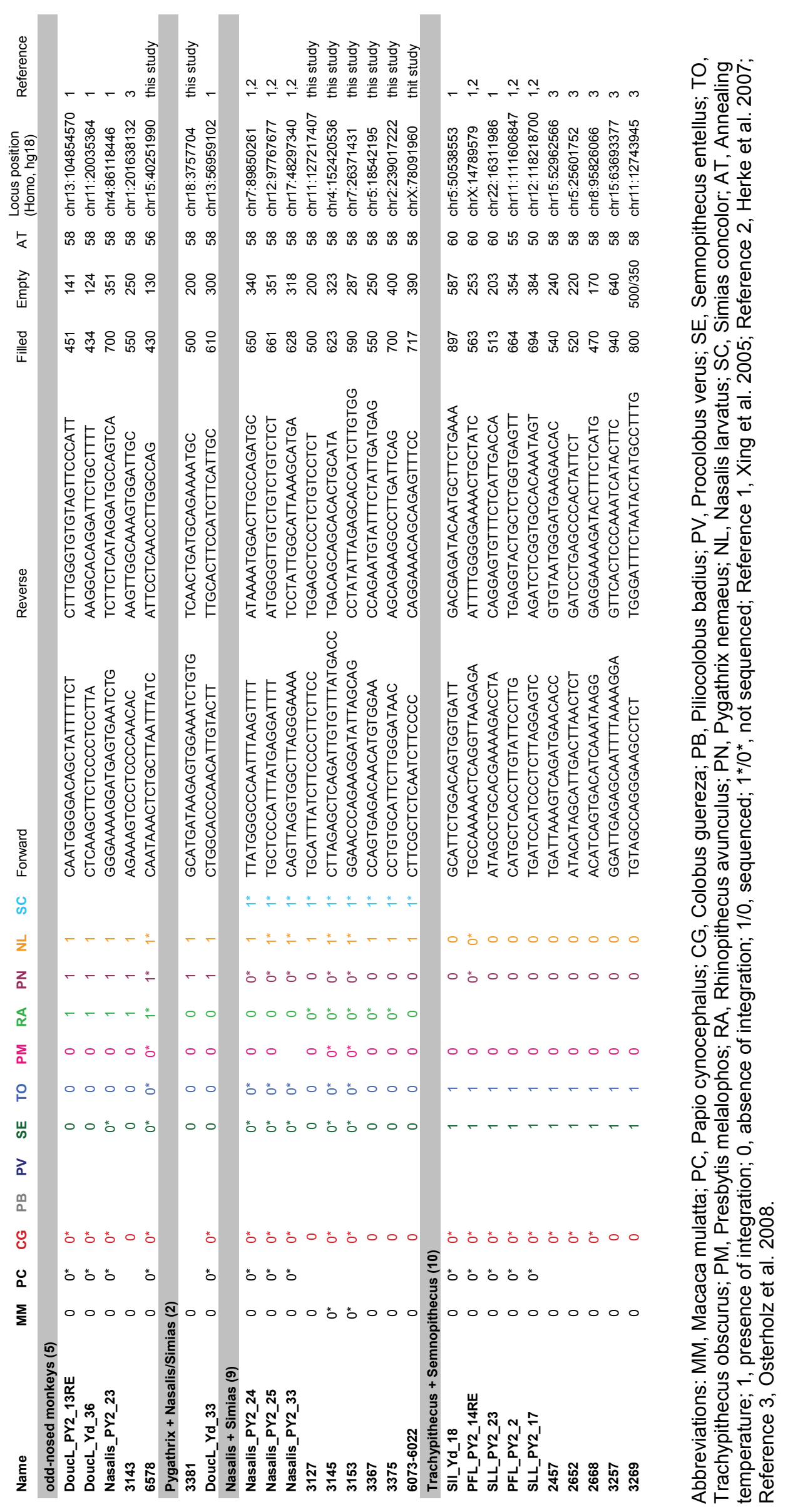




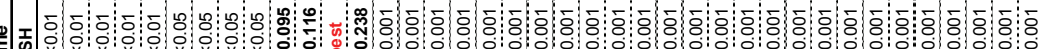

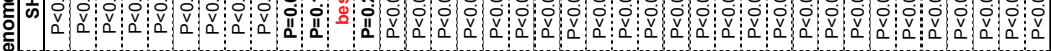
๘ -

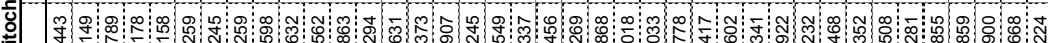

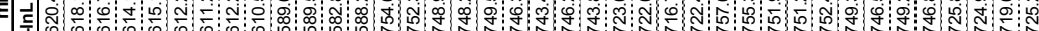

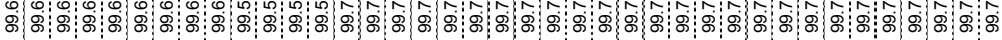

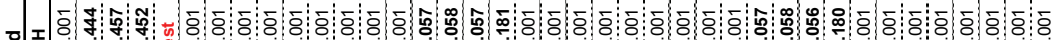

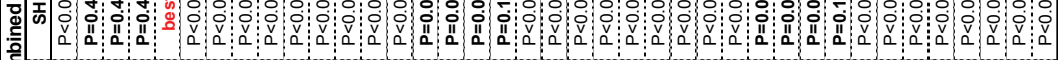

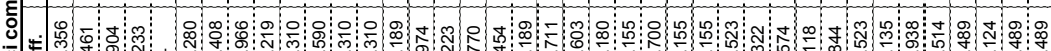

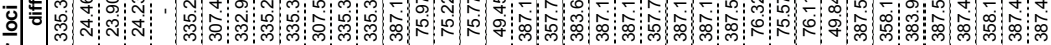

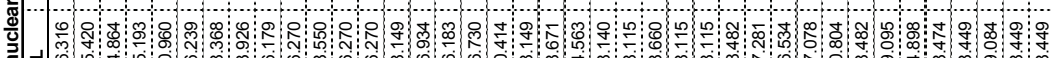
可: \&

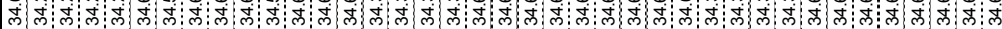

ซ

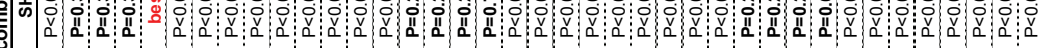

-

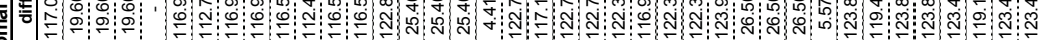

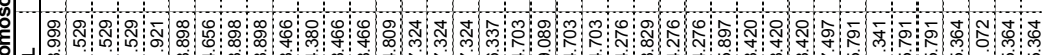

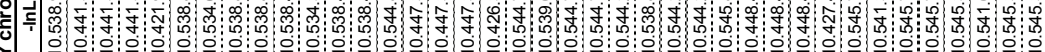

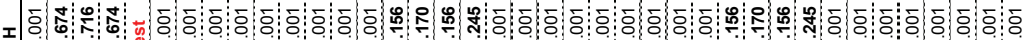

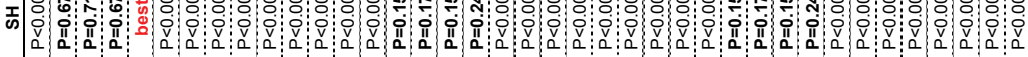

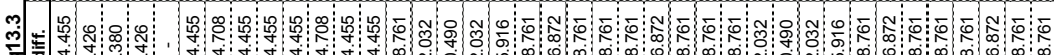

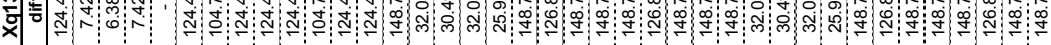

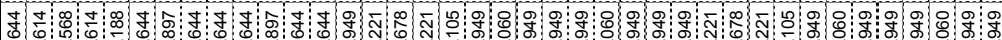

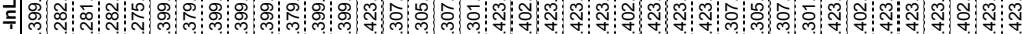

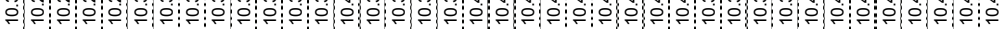

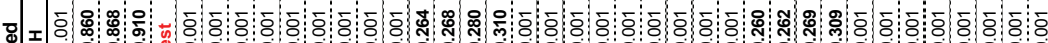
W. o

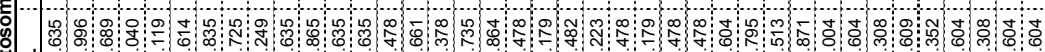

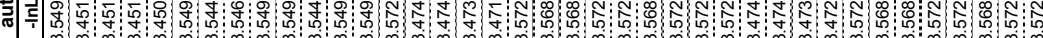
๗ூ)

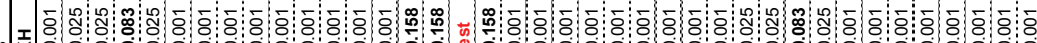

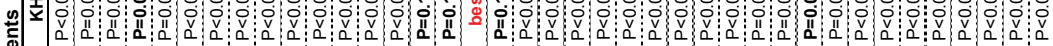

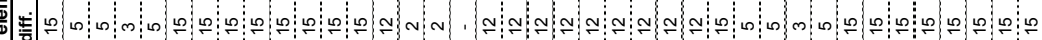

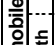

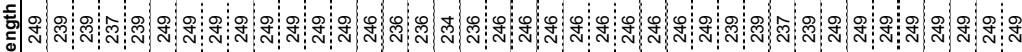

言商:

ग:

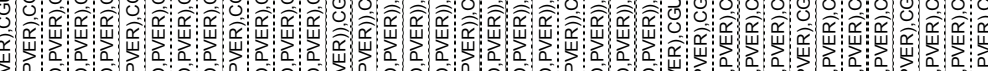

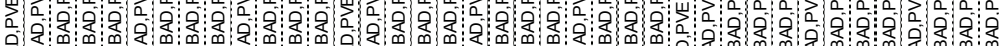

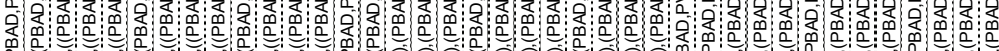

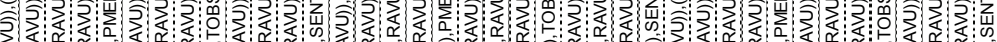

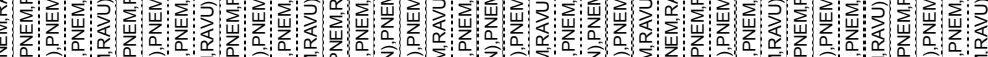

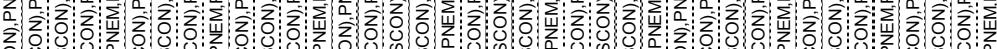
$\omega n$ w

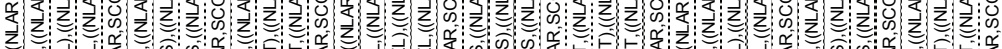

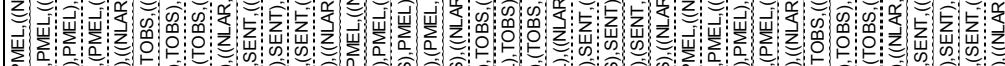

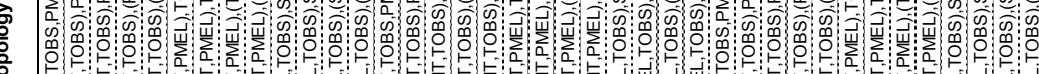

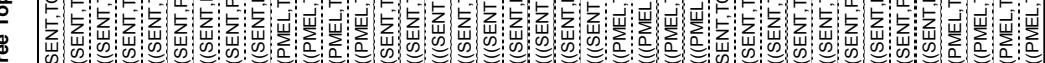


Additional file 3: Additional Figure 1. Single-locus phylogenetic trees (80\% majority rule).

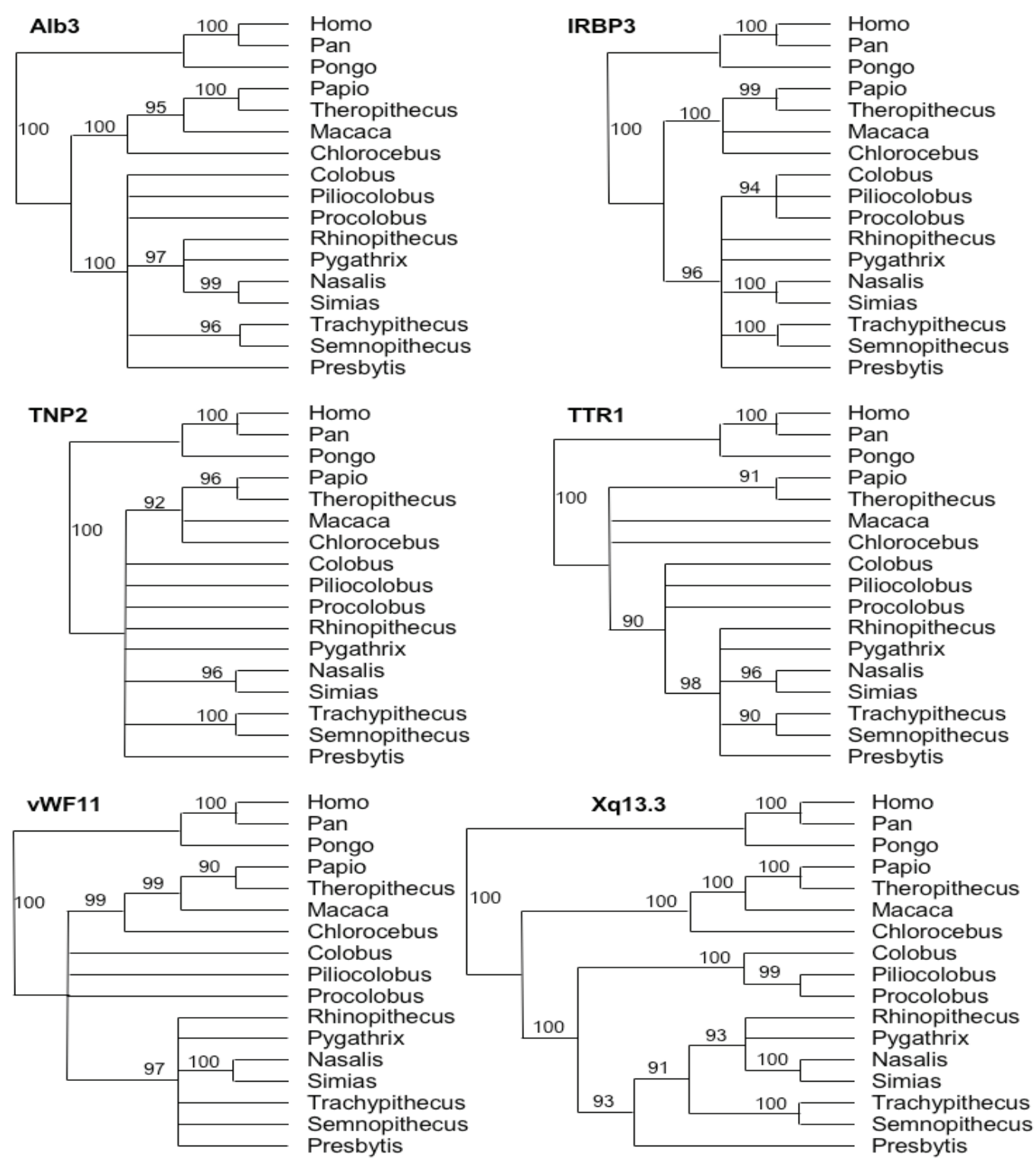



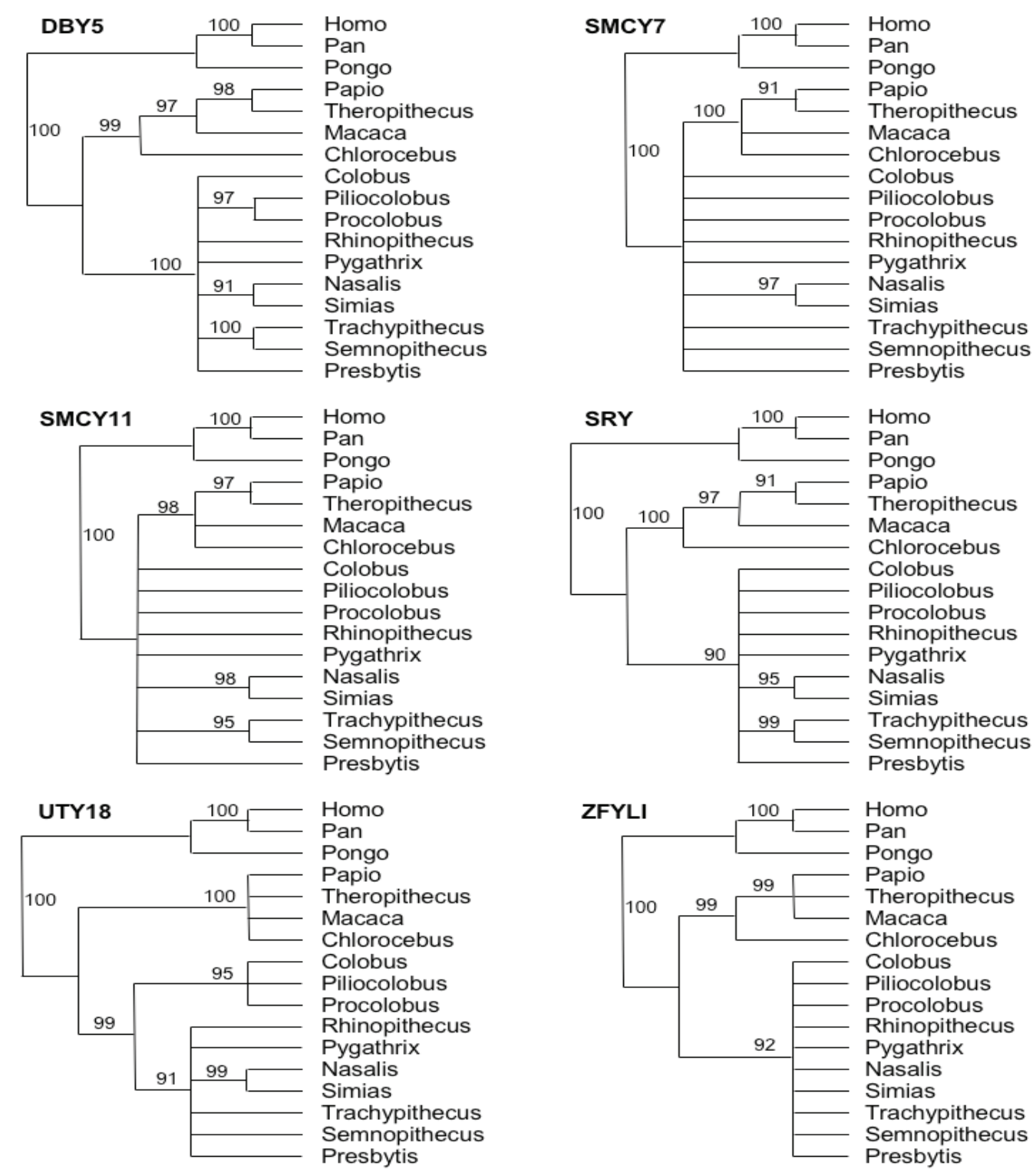
Additional file 4: Additional Figure 2. Nucleotide composition of both mitochondrial and the combined nuclear datasets.
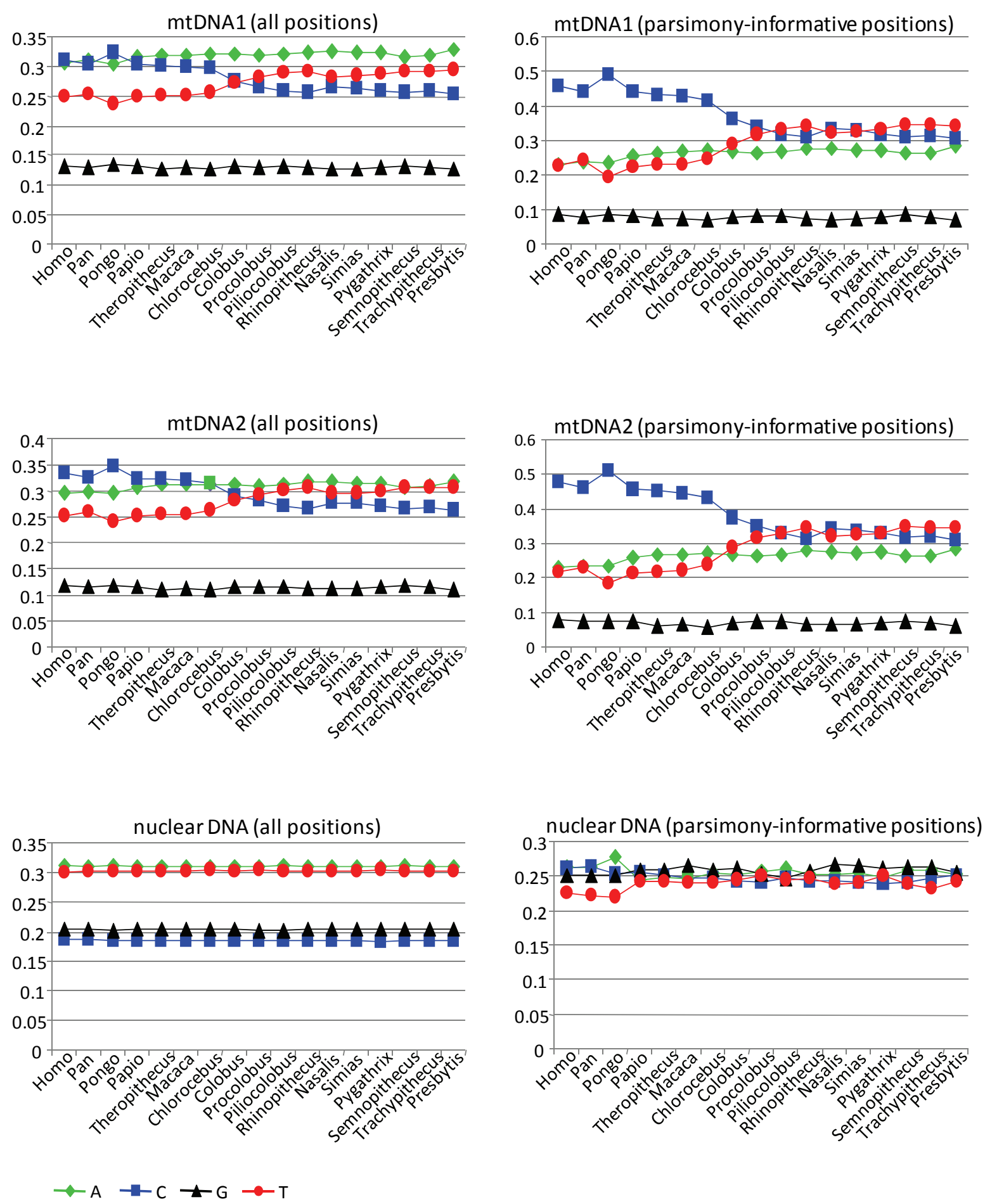
Additional file 5: Additional Figure 3. Phylograms based on the mitochondrial (mtDNA2) and combined nuclear datasets.

Phylogram based on the mitochondrial dataset (mtDNA2):

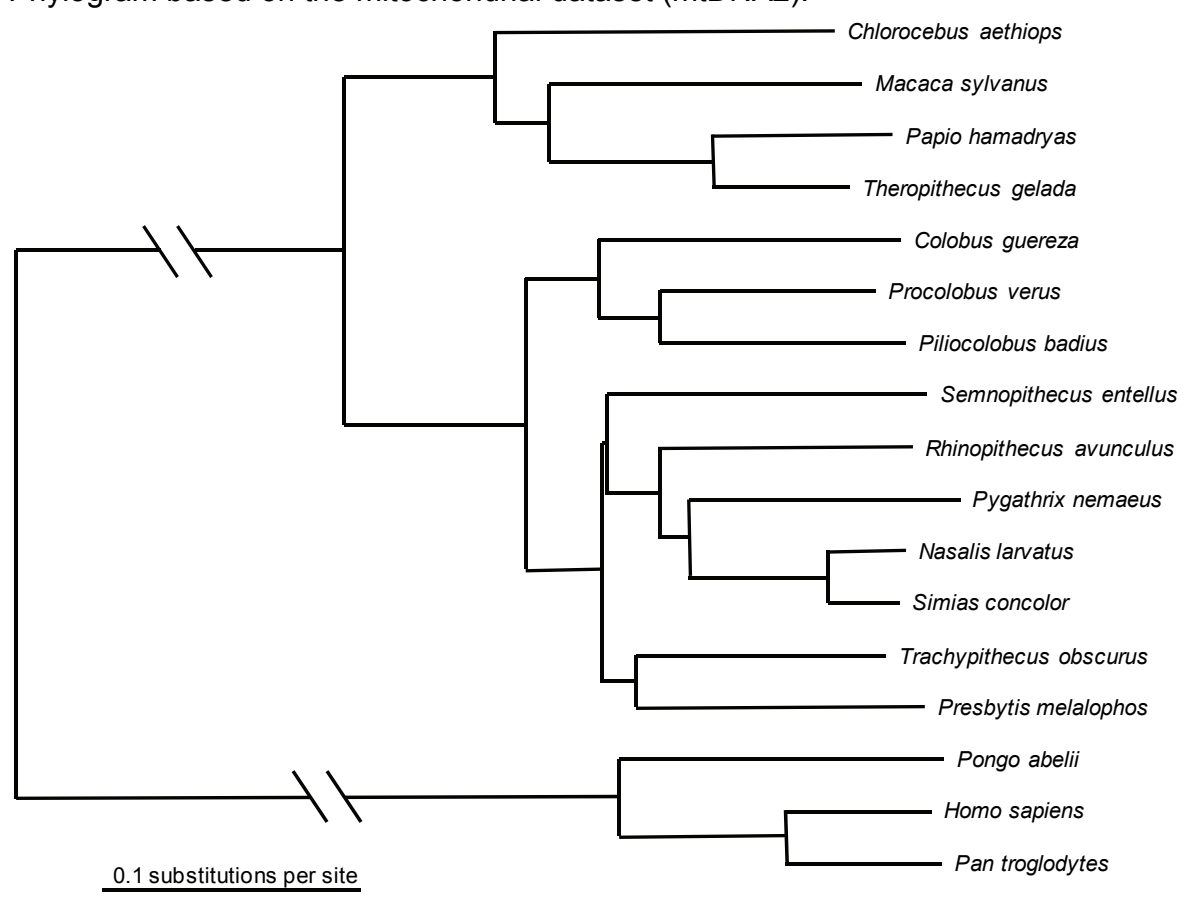

Phylogram based on the combined nuclear dataset:

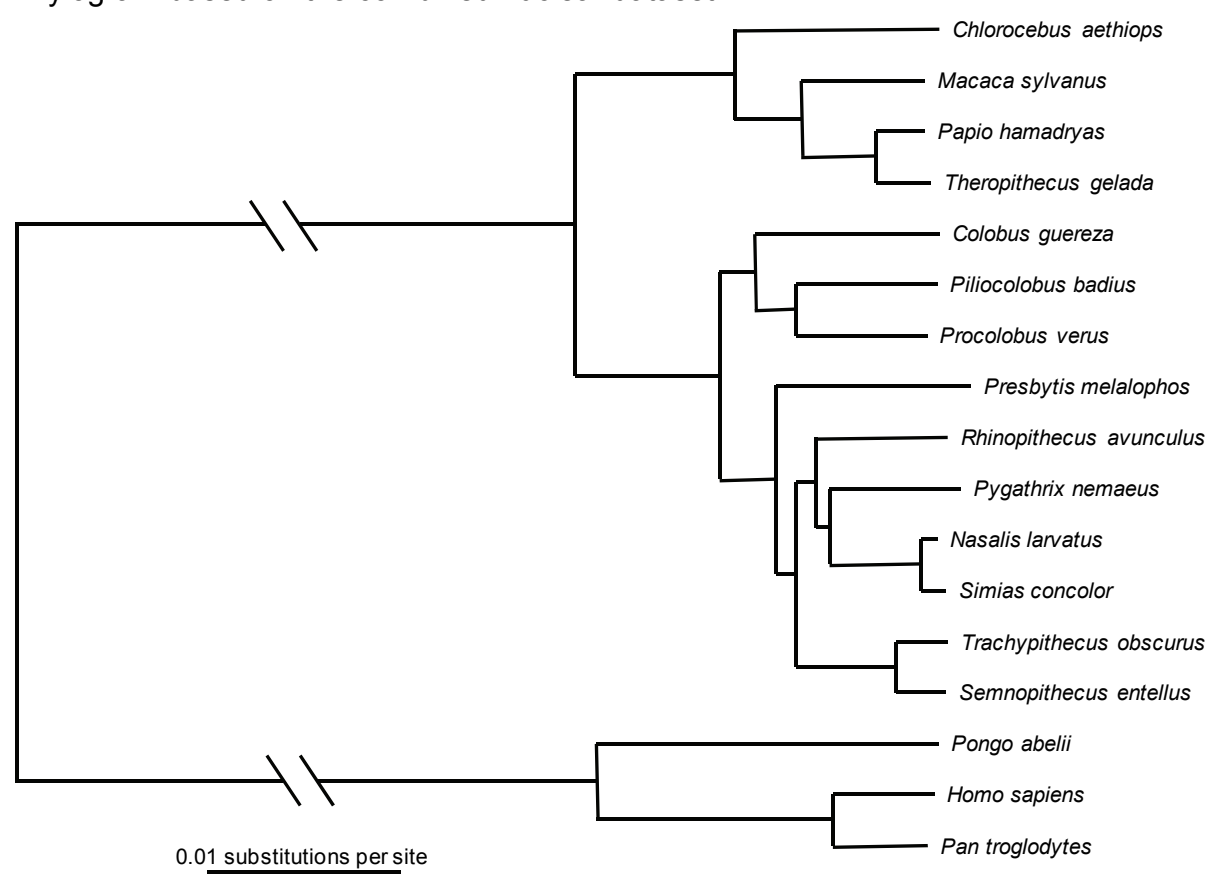


Additional file 6: Additional Table 3. Divergence ages in mya estimated for each locus separately.

\begin{tabular}{lcccc}
\hline & Alb3 & IRBP3 & TNP2 & TTR1 \\
\hline cercopithecoids-hominoids & $23.45(21.17-25.52)$ & $24.09(22.08-26.24)$ & $23.57(21.41-25.77)$ & $23.49(21.33-25.77)$ \\
Pongo-Homo/Pan & $13.81(12.57-14.91)$ & $13.87(12.74-14.97)$ & $13.95(12.79-15.09)$ & $13.98(12.87-15.15)$ \\
Homo-Pan & $6.48(5.87-7.04)$ & $6.41(5.83-6.99)$ & $6.48(5.92-7.07)$ & $6.44(5.87-7.03)$ \\
cercopithecines-colobines & $15.45(14.31-16.56)$ & $15.15(14.04-16.24)$ & $15.03(13.83-16.16)$ & $15.37(14.26-16.49)$ \\
Chlorocebus -other cercopithecines & $8.93(5.98-12.12)$ & $8.49(6.17-10.75)$ & $9.42(6.07-13.38)$ & $11.76(8.09-15.12)$ \\
Macaca-Theropithecus/Papio & $7.12(4.73-9.86)$ & $6.03(4.46-7.83)$ & $7.27(4.60-10.65)$ & $9.54(5.85-13.30)$ \\
Theropithecus-Papio & $3.88(3.28-4.47)$ & $3.85(3.28-4.41)$ & $3.93(3.37-4.51)$ & $3.95(3.36-4.54)$ \\
Colobus -other colobines & $10.87(8.86-12.95)$ & $10.87(9.28-12.67)$ & $13.49(10.97-15.56)$ & $12.96(10.67-15.10)$ \\
Procolobus/Piliocolobus -Asian colobines & $10.26(8.87-12.14)$ & $10.41(8.84-12.02)$ & $12.14(9.79-14.75)$ & $12.26(9.95-14.41)$ \\
Procolobus-Piliocolobus & $7.67(3.70-11.07)$ & $7.62(5.00-10.09)$ & $6.19(2.51-10.11)$ & $7.52(3.50-11.26)$ \\
Asian colobines & $8.73(7.61-9.81)$ & $8.98(7.88-9.95)$ & $9.01(7.93-10.10)$ & $8.56(7.42-9.70)$ \\
Semnopithecus-Trachypithecus & $2.99(0.61-5.81)$ & $3.24(1.58-4.94)$ & $1.76(0.21-3.74)$ & $3.37(0.67-6.67)$ \\
Presbytis -odd-nosed monkeys & $8.04(6.26-9.46)$ & $8.09(6.66-9.39)$ & $7.94(5.90-9.78)$ & $7.22(4.77-9.18)$ \\
odd-nosed monkeys & $5.20(2.96-7.43)$ & $5.77(4.10-7.45)$ & $5.69(3.21-8.16)$ & $6.26(3.75-8.50)$ \\
Pygathrix-Nasalis/Simias & $3.13(1.22-5.33)$ & $5.41(3.76-7.09)$ & $4.63(2.24-7.20)$ & $5.41(2.88-7.86)$ \\
Nasalis-Simias & $0.36(0.10-1.00)$ & $2.43(1.07-3.74)$ & $1.10(0.03-2.58)$ & $0.51(0.00-1.58)$ \\
\hline
\end{tabular}

\begin{tabular}{lcccc}
\hline & vWF11 & Xq13.3 & DBY5 & SMCY7 \\
\hline cercopithecoids-hominoids & $23.44(21.24-25.73)$ & $24.12(22.04-26.28)$ & $23.63(21.44-25.80)$ & $22.93(20.53-25.23)$ \\
Pongo-Homo/Pan & $13.93(12.72-15.01)$ & $13.82(12.73-14.97)$ & $13.94(12.74-15.02)$ & $14.02(12.89-15.21)$ \\
Homo-Pan & $6.41(5.82-7.01)$ & $6.43(5.86-7.02)$ & $6.40(5.83-6.98)$ & $6.44(5.83-7.03)$ \\
cercopithecines-colobines & $15.48(14.32-16.56)$ & $15.47(14.40-16.56)$ & $15.43(14.32-16.51)$ & $15.44(14.35-16.63)$ \\
Chlorocebus -other cercopithecines & $10.33(7.16-13.40)$ & $9.78(7.37-12.50)$ & $9.60(6.72-12.90)$ & $9.19(5.25-13.01)$ \\
Macaca-Theropithecus/Papio & $5.67(3.87-7.78)$ & $6.65(4.86-8.84)$ & $7.23(4.82-10-04)$ & $6.36(4.07-9.45)$ \\
Theropithecus-Papio & $3.96(3.39-4.53)$ & $3.83(3.25-4.39)$ & $3.88(3.27-4.43)$ & $3.90(3.35-4.49)$ \\
Colobus -other colobines & $13.46(11.35-15.48)$ & $10.80(9.14-12.34)$ & $10.15(8.40-12.16)$ & $11.77(9.10-14.51)$ \\
Procolobus/Piliocolobus -Asian colobines & $12.46(10.35-14.67)$ & $10.57(9.01-12.14)$ & $9.20(7.79-10.66)$ & $10.04(8.01-12.48)$ \\
Procolobus-Piliocolobus & $10.22(7.56-13.02)$ & $6.94(4.17-9.63)$ & $2.61(0.27-3.13)$ & $2.40(0.02-6.34)$ \\
Asian colobines & $8.56(7.43-9.64)$ & $8.35(7.25-9.36)$ & $8.67(7.58-9.76)$ & $8.74(7.59-9.86)$ \\
Semnopithecus-Trachypithecus & $6.64(3.07-9.22)$ & $2.02(0.70-3.64)$ & $1.76(0.26-3.56)$ & $3.21(0.28-7.04)$ \\
Presbytis -odd-nosed monkeys & $7.62(5.72-9.33)$ & $7.96(6.71-9.27)$ & $7.74(5.92-9.49)$ & $7.25(4.31-9.84)$ \\
odd-nosed monkeys & $6.75(4.66-8.91)$ & $6.28(4.26-8.08)$ & $6.06(3.99-8.36)$ & $5.53(2.53-7.84)$ \\
Pygathrix-Nasalis/Simias & $4.12(1.87-6.37)$ & $5.93(3.87-7.83)$ & $5.33(3.07-7.55)$ & $4.60(1.59-7.90)$ \\
Nasalis-Simias & $0.72(0.01-1.81)$ & $1.13(0.28-2.23)$ & $1.56(0.27-3.13)$ & $1.22(0.02-3.13)$ \\
\hline
\end{tabular}

\begin{tabular}{lcccc}
\hline & SMCY11 & SRY & UTY18 & ZFYLI \\
\hline cercopithecoids-hominoids & $23.15(21.04-25.45$ & $23.34(21.27-25.63)$ & $23.04(20.85-25.35)$ & $23.23(21.11-25.43)$ \\
Pongo-Homo/Pan & $13.94(12.77-15.10)$ & $13.93(12.82-15.06)$ & $14.00(12.83-15.15)$ & $14.13(12.99-15.24)$ \\
Homo-Pan & $6.50(5.93-7.07)$ & $6.47(5.86-7.01)$ & $6.46(5.90-7.08)$ & $6.39(5.79-6.97)$ \\
cercopithecines-colobines & $15.32(14.20-16.46)$ & $15.44(14.35-16.56)$ & $15.47(14.26-16.54)$ & $15.38(14.28-16.51)$ \\
Chlorocebus -other cercopithecines & $8.62(5.16-12.41)$ & $9.03(6.20-12.07)$ & $8.57(4.96-12.29)$ & $10.20(6.86-13.25)$ \\
Macaca-Theropithecus/Papio & $7.15(4.22-10.40)$ & $5.98(4.18-8.23)$ & $5.59(3.64-8.22)$ & $6.07(3.96-8.61)$ \\
Theropithecus-Papio & $3.91(3.35-4.53)$ & $3.84(3.26-4.41)$ & $3.92(3.34-4.49)$ & $3.92(3.32-4.46)$ \\
Colobus -other colobines & $11.37(9.11-13.79)$ & $10.97(8.99-13.24)$ & $11.46(9.24-13.87)$ & $11.96(9.73-14.26)$ \\
Procolobus/Piliocolobus -Asian colobines & $10.48(8.54-12.61)$ & $10.32(8.40-12.28)$ & $10.76(8.72-13.02)$ & $11.24(9.19-13.52)$ \\
Procolobus-Piliocolobus & $5.33(1.75-8.90)$ & $4.33(1.09-8.11)$ & $3.86(0.35-8.61)$ & $6.46(2.72-10.35)$ \\
Asian colobines & $8.80(7.67-9.85)$ & $8.66(7.62-9.78)$ & $8.58(7.46-9.74)$ & $8.67(7.55-9.77)$ \\
Semnopithecus-Trachypithecus & $3.10(0.65-6.03)$ & $3.37(1.14-5.86)$ & $3.03(0.29-6.57)$ & $2.66(0.21-5.89)$ \\
Presbytis -odd-nosed monkeys & $7.81(5.74-9.60)$ & $7.69(5.71-9.38)$ & $7.34(4.96-9.41)$ & $7.81(6.02-9.52)$ \\
odd-nosed monkeys & $6.43(3.79-8.71)$ & $6.12(3.72-8.33)$ & $5.92(3.22-8.60)$ & $6.53(4.11-8.65)$ \\
Pygathrix-Nasalis/Simias & $5.31(2.61-7.87)$ & $5.21(2.57-7.78)$ & $4.84(2.03-7.68)$ & $4.14(1.84-6.49)$ \\
Nasalis-Simias & $1.53(0.15-3.31)$ & $1.12(0.03-2.67)$ & $0.61(0.00-1.91)$ & $1.00(0.02-2.37)$ \\
\hline
\end{tabular}


Additional file 7: Additional Table 4. Uncorrected pairwise nucleotide differences for each locus

\begin{tabular}{|c|c|c|c|c|c|c|c|c|c|}
\hline Alb3 & CGUE & PBAD & PVER & RAVU & PNEM & NLAR & SCON & PMEL & TOBS \\
\hline PBAD & 0.0156 & & & & & & & & \\
\hline PVER & 0.0147 & 0.0113 & & & & & & & \\
\hline RAVU & 0.0233 & 0.0182 & 0.0190 & & & & & & \\
\hline PNEM & 0.0190 & 0.0138 & 0.0147 & 0.0112 & & & & & \\
\hline NLAR & 0.0216 & 0.0165 & 0.0173 & 0.0138 & 0.0061 & & & & \\
\hline SCON & 0.0216 & 0.0165 & 0.0173 & 0.0138 & 0.0061 & 0.0000 & & & \\
\hline PMEL & 0.0242 & 0.0208 & 0.0182 & 0.0233 & 0.0190 & 0.0216 & 0.0216 & & \\
\hline TOBS & 0.0190 & 0.0138 & 0.0147 & 0.0182 & 0.0138 & 0.0147 & 0.0147 & 0.0190 & \\
\hline SENT & 0.0164 & 0.0130 & 0.0121 & 0.0156 & 0.0112 & 0.0138 & 0.0138 & 0.0164 & 0.0043 \\
\hline IRBP3 & CGUE & PBAD & PVER & RAVU & PNEM & NLAR & SCON & PMEL & TOBS \\
\hline PBAD & 0.0137 & & & & & & & & \\
\hline PVER & 0.0189 & 0.0150 & & & & & & & \\
\hline RAVU & 0.0207 & 0.0182 & 0.0214 & & & & & & \\
\hline PNEM & 0.0207 & 0.0182 & 0.0208 & 0.0117 & & & & & \\
\hline NLAR & 0.0214 & 0.0202 & 0.0234 & 0.0110 & 0.0149 & & & & \\
\hline SCON & 0.0214 & 0.0202 & 0.0234 & 0.0110 & 0.0149 & 0.0065 & & & \\
\hline PMEL & 0.0221 & 0.0209 & 0.0241 & 0.0188 & 0.0163 & 0.0208 & 0.0221 & & \\
\hline TOBS & 0.0233 & 0.0195 & 0.0240 & 0.0156 & 0.0195 & 0.0169 & 0.0169 & 0.0221 & \\
\hline SENT & 0.0246 & 0.0208 & 0.0253 & 0.0182 & 0.0221 & 0.0195 & 0.0195 & 0.0234 & 0.0078 \\
\hline TNP2 & CGUE & PBAD & PVER & RAVU & PNEM & NLAR & SCON & PMEL & TOBS \\
\hline PBAD & 0.0202 & & & & & & & & \\
\hline PVER & 0.0233 & 0.0124 & & & & & & & \\
\hline RAVU & 0.0279 & 0.0202 & 0.0233 & & & & & & \\
\hline PNEM & 0.0264 & 0.0172 & 0.0202 & 0.0109 & & & & & \\
\hline NLAR & 0.0264 & 0.0186 & 0.0217 & 0.0109 & 0.0093 & & & & \\
\hline SCON & 0.0279 & 0.0202 & 0.0234 & 0.0124 & 0.0109 & 0.0016 & & & \\
\hline PMEL & 0.0326 & 0.0249 & 0.0279 & 0.0202 & 0.0186 & 0.0186 & 0.0202 & & \\
\hline TOBS & 0.0326 & 0.0249 & 0.0279 & 0.0202 & 0.0186 & 0.0186 & 0.0202 & 0.0248 & \\
\hline SENT & 0.0326 & 0.0249 & 0.0279 & 0.0202 & 0.0186 & 0.0186 & 0.0202 & 0.0248 & 0.0031 \\
\hline TTR1 & CGUE & PBAD & PVER & RAVU & PNEM & NLAR & SCON & PMEL & TOBS \\
\hline PBAD & 0.0214 & & & & & & & & \\
\hline PVER & 0.0248 & 0.0169 & & & & & & & \\
\hline RAVU & 0.0281 & 0.0214 & 0.0293 & & & & & & \\
\hline PNEM & 0.0293 & 0.0225 & 0.0304 & 0.0146 & & & & & \\
\hline NLAR & 0.0259 & 0.0214 & 0.0293 & 0.0113 & 0.0146 & & & & \\
\hline SCON & 0.0259 & 0.0214 & 0.0293 & 0.0113 & 0.0146 & 0.0000 & & & \\
\hline PMEL & 0.0260 & 0.0192 & 0.0271 & 0.0090 & 0.0101 & 0.0090 & 0.0090 & & \\
\hline TOBS & 0.0281 & 0.0214 & 0.0293 & 0.0113 & 0.0146 & 0.0113 & 0.0113 & 0.0090 & \\
\hline SENT & 0.0259 & 0.0192 & 0.0271 & 0.0090 & 0.0124 & 0.0090 & 0.0090 & 0.0068 & 0.0045 \\
\hline
\end{tabular}




\begin{tabular}{|c|c|c|c|c|c|c|c|c|c|}
\hline vWF11 & CGUE & PBAD & PVER & RAVU & PNEM & NLAR & SCON & PMEL & TOBS \\
\hline PBAD & 0.0332 & & & & & & & & \\
\hline PVER & 0.0277 & 0.0332 & & & & & & & \\
\hline RAVU & 0.0332 & 0.0354 & 0.0376 & & & & & & \\
\hline PNEM & 0.0322 & 0.0366 & 0.0388 & 0.0211 & & & & & \\
\hline NLAR & 0.0366 & 0.0367 & 0.0366 & 0.0166 & 0.0134 & & & & \\
\hline SCON & 0.0376 & 0.0376 & 0.0376 & 0.0177 & 0.0144 & 0.0011 & & & \\
\hline PMEL & 0.0300 & 0.0311 & 0.0299 & 0.0177 & 0.0167 & 0.0167 & 0.0155 & & \\
\hline TOBS & 0.0277 & 0.0265 & 0.0298 & 0.0144 & 0.0144 & 0.0133 & 0.0144 & 0.0100 & \\
\hline SENT & 0.0321 & 0.0320 & 0.0320 & 0.0188 & 0.0166 & 0.0155 & 0.0166 & 0.0122 & 0.0111 \\
\hline Xq13.3 & CGUE & PBAD & PVER & RAVU & PNEM & NLAR & SCON & PMEL & TOBS \\
\hline PBAD & 0.0157 & & & & & & & & \\
\hline PVER & 0.0166 & 0.0142 & & & & & & & \\
\hline RAVU & 0.0180 & 0.0185 & 0.0199 & & & & & & \\
\hline PNEM & 0.0192 & 0.0202 & 0.0206 & 0.0126 & & & & & \\
\hline NLAR & 0.0161 & 0.0171 & 0.0185 & 0.0100 & 0.0111 & & & & \\
\hline SCON & 0.0164 & 0.0173 & 0.0187 & 0.0102 & 0.0114 & 0.0017 & & & \\
\hline PMEL & 0.0187 & 0.0197 & 0.0202 & 0.0149 & 0.0164 & 0.0133 & 0.0135 & & \\
\hline TOBS & 0.0195 & 0.0204 & 0.0218 & 0.0142 & 0.0149 & 0.0123 & 0.0126 & 0.0154 & \\
\hline SENT & 0.0185 & 0.0195 & 0.0209 & 0.0133 & 0.0140 & 0.0114 & 0.0116 & 0.0145 & 0.0033 \\
\hline DBY5 & CGUE & PBAD & PVER & RAVU & PNEM & NLAR & SCON & PMEL & TOBS \\
\hline PBAD & 0.0363 & & & & & & & & \\
\hline PVER & 0.0287 & 0.0076 & & & & & & & \\
\hline RAVU & 0.0272 & 0.0242 & 0.0166 & & & & & & \\
\hline PNEM & 0.0257 & 0.0227 & 0.0181 & 0.0166 & & & & & \\
\hline NLAR & 0.0302 & 0.0227 & 0.0151 & 0.0181 & 0.0196 & & & & \\
\hline SCON & 0.0287 & 0.0181 & 0.0106 & 0.0166 & 0.0181 & 0.0045 & & & \\
\hline PMEL & 0.0317 & 0.0302 & 0.0242 & 0.0227 & 0.0212 & 0.0257 & 0.0242 & & \\
\hline TOBS & 0.0317 & 0.0257 & 0.0212 & 0.0227 & 0.0242 & 0.0257 & 0.0212 & 0.0272 & \\
\hline SENT & 0.0332 & 0.0302 & 0.0227 & 0.0242 & 0.0257 & 0.0272 & 0.0227 & 0.0287 & 0.0045 \\
\hline SMCY7 & CGUE & PBAD & PVER & RAVU & PNEM & NLAR & SCON & PMEL & TOBS \\
\hline PBAD & 0.0162 & & & & & & & & \\
\hline PVER & 0.0139 & 0.0023 & & & & & & & \\
\hline RAVU & 0.0231 & 0.0208 & 0.0185 & & & & & & \\
\hline PNEM & 0.0139 & 0.0116 & 0.0092 & 0.0139 & & & & & \\
\hline NLAR & 0.0208 & 0.0139 & 0.0116 & 0.0208 & 0.0116 & & & & \\
\hline SCON & 0.0231 & 0.0162 & 0.0139 & 0.0231 & 0.0139 & 0.0023 & & & \\
\hline PMEL & 0.0162 & 0.0139 & 0.0116 & 0.0185 & 0.0116 & 0.0185 & 0.0208 & & \\
\hline TOBS & 0.0162 & 0.0139 & 0.0116 & 0.0162 & 0.0069 & 0.0139 & 0.0162 & 0.0139 & \\
\hline SENT & 0.0162 & 0.0139 & 0.0116 & 0.0162 & 0.0069 & 0.0139 & 0.0162 & 0.0139 & 0.004 \\
\hline
\end{tabular}




\begin{tabular}{|c|c|c|c|c|c|c|c|c|c|}
\hline SMCY11 & CGUE & PBAD & PVER & RAVU & PNEM & NLAR & SCON & PMEL & TOBS \\
\hline PBAD & 0.0154 & & & & & & & & \\
\hline PVER & 0.0055 & 0.0166 & & & & & & & \\
\hline RAVU & 0.0122 & 0.0351 & 0.0185 & & & & & & \\
\hline PNEM & 0.0147 & 0.0314 & 0.0148 & 0.0148 & & & & & \\
\hline NLAR & 0.0169 & 0.0333 & 0.0166 & 0.0166 & 0.0129 & & & & \\
\hline SCON & 0.0193 & 0.0370 & 0.0203 & 0.0203 & 0.0166 & 0.0037 & & & \\
\hline PMEL & 0.0227 & 0.0447 & 0.0296 & 0.0296 & 0.0296 & 0.0314 & 0.0351 & & \\
\hline TOBS & 0.0197 & 0.0388 & 0.0222 & 0.0222 & 0.0185 & 0.0203 & 0.0240 & 0.0333 & \\
\hline SENT & 0.0197 & 0.0351 & 0.0185 & 0.0185 & 0.0148 & 0.0166 & 0.0203 & 0.0333 & 0.0074 \\
\hline SRY & CGUE & PBAD & PVER & RAVU & PNEM & NLAR & SCON & PMEL & TOBS \\
\hline PBAD & 0.0143 & & & & & & & & \\
\hline PVER & 0.0078 & 0.0065 & & & & & & & \\
\hline RAVU & 0.0207 & 0.0220 & 0.0155 & & & & & & \\
\hline PNEM & 0.0168 & 0.0181 & 0.0117 & 0.0143 & & & & & \\
\hline NLAR & 0.0155 & 0.0168 & 0.0104 & 0.0130 & 0.0091 & & & & \\
\hline SCON & 0.0168 & 0.0181 & 0.0117 & 0.0143 & 0.0104 & 0.0013 & & & \\
\hline PMEL & 0.0194 & 0.0207 & 0.0143 & 0.0220 & 0.0181 & 0.0168 & 0.0181 & & \\
\hline TOBS & 0.0207 & 0.0220 & 0.0155 & 0.0181 & 0.0143 & 0.0130 & 0.0143 & 0.0220 & \\
\hline SENT & 0.0233 & 0.0220 & 0.0181 & 0.0207 & 0.0168 & 0.0155 & 0.0168 & 0.0246 & 0.0078 \\
\hline UTY18 & CGUE & PBAD & PVER & RAVU & PNEM & NLAR & SCON & PMLE & TOBS \\
\hline PBAD & 0.0121 & & & & & & & & \\
\hline PVER & 0.0073 & 0.0048 & & & & & & & \\
\hline RAVU & 0.0145 & 0.0145 & 0.0097 & & & & & & \\
\hline PNEM & 0.0205 & 0.0205 & 0.0157 & 0.0109 & & & & & \\
\hline NLAR & 0.0181 & 0.0181 & 0.0133 & 0.0085 & 0.0133 & & & & \\
\hline SCON & 0.0181 & 0.0181 & 0.0133 & 0.0085 & 0.0133 & 0.0000 & & & \\
\hline PMEL & 0.0230 & 0.0230 & 0.0181 & 0.0133 & 0.0205 & 0.0169 & 0.0169 & & \\
\hline TOBS & 0.0169 & 0.0169 & 0.0121 & 0.0073 & 0.0121 & 0.0085 & 0.0085 & 0.0157 & \\
\hline SENT & 0.0181 & 0.0181 & 0.0133 & 0.0060 & 0.0133 & 0.0097 & 0.0097 & 0.0133 & 0.0036 \\
\hline ZFYLI & CGUE & PBAD & PVER & RAVU & PNEM & NLAR & SCON & PMEL & TOBS \\
\hline PBAD & 0.0118 & & & & & & & & \\
\hline PVER & 0.0132 & 0.0118 & & & & & & & \\
\hline RAVU & 0.0191 & 0.0206 & 0.0206 & & & & & & \\
\hline PNEM & 0.0221 & 0.0235 & 0.0235 & 0.0147 & & & & & \\
\hline NLAR & 0.0191 & 0.0206 & 0.0206 & 0.0147 & 0.0088 & & & & \\
\hline SCON & 0.0177 & 0.0191 & 0.0191 & 0.0132 & 0.0103 & 0.0015 & & & \\
\hline PMEL & 0.0206 & 0.0221 & 0.0221 & 0.0191 & 0.0221 & 0.0221 & 0.0206 & & \\
\hline TOBS & 0.0162 & 0.0177 & 0.0177 & 0.0088 & 0.0118 & 0.0117 & 0.0103 & 0.0162 & \\
\hline SENT & 0.0162 & 0.0177 & 0.0177 & 0.0088 & 0.0118 & 0.0118 & 0.0103 & 0.0162 & 0.0029 \\
\hline
\end{tabular}




$\begin{array}{llllllllll}\text { mtDNA1 } & \text { CGUE } & \text { PBAD } & \text { PVER } & \text { RAVU } & \text { PNEM } & \text { NLAR } & \text { SCON } & \text { PMEL } & \text { TOBS } \\ \text { PBAD } & 0.1361 & & & & & & & & \\ \text { PVER } & 0.1318 & 0.1162 & & & & & & & \\ \text { RAVU } & 0.1476 & 0.1449 & 0.1402 & & & & & & \\ \text { PNEM } & 0.1497 & 0.1497 & 0.1457 & 0.1210 & & & & & \\ \text { NLAR } & 0.1455 & 0.1465 & 0.1381 & 0.1174 & 0.1223 & & & & \\ \text { SCON } & 0.1455 & 0.1418 & 0.1400 & 0.1164 & 0.1211 & 0.0458 & & & \\ \text { PMEL } & 0.1540 & 0.1501 & 0.1477 & 0.1329 & 0.1389 & 0.1337 & 0.1322 & & \\ \text { TOBS } & 0.1464 & 0.1461 & 0.1420 & 0.1302 & 0.1367 & 0.1307 & 0.1323 & 0.1292 & \\ \text { SENT } & 0.1548 & 0.1505 & 0.1495 & 0.1350 & 0.1431 & 0.1347 & 0.1355 & 0.1405 & 0.1353 \\ & & & & & & & & & \\ \text { mtDNA2 } & \text { CGUE } & \text { PBAD } & \text { PVER } & \text { RAVU } & \text { PNEM } & \text { NLAR } & \text { SCON } & \text { PMEL } & \text { TOBS } \\ \text { PBAD } & 0.1565 & & & & & & & & \\ \text { PVER } & 0.1523 & 0.1363 & & & & & & & \\ \text { RAVU } & 0.1673 & 0.1659 & 0.1593 & & & & & & \\ \text { PNEM } & 0.1689 & 0.1712 & 0.1664 & 0.1399 & & & & & \\ \text { NLAR } & 0.1660 & 0.1682 & 0.1576 & 0.1352 & 0.1429 & & & & \\ \text { SCON } & 0.1656 & 0.1630 & 0.1601 & 0.1354 & 0.1415 & 0.0529 & & & \\ \text { PMEL } & 0.1758 & 0.1726 & 0.1694 & 0.1521 & 0.1592 & 0.1541 & 0.1524 & & \\ \text { TOBS } & 0.1676 & 0.1684 & 0.1632 & 0.1494 & 0.1568 & 0.1480 & 0.1509 & 0.1486 & \\ \text { SENT } & 0.1760 & 0.1713 & 0.1712 & 0.1532 & 0.1648 & 0.1539 & 0.1560 & 0.1612 & 0.1537\end{array}$


Additional file 8: Additional Table 5. Locus-specific information including alignment length, number of variable sites, selected substitution model and estimated evolutionary rates.

\begin{tabular}{lcccc}
\hline Locus & $\begin{array}{c}\text { Alignment with / } \\
\text { without indels }\end{array}$ & $\begin{array}{c}\text { Variable sites / } \\
\text { parsimony- } \\
\text { informative sites }\end{array}$ & $\begin{array}{c}\text { Substitution } \\
\text { model (AIC) }\end{array}$ & $\begin{array}{c}\text { Estimated } \\
\text { evolutionary rates }\end{array}$ \\
\hline ALB3 & $1204 / 1157$ & $182 / 93$ & TVM + I & 0.902 \\
IRBP3 & $1615 / 1543$ & $263 / 114$ & K81uf + G & 0.848 \\
TNP2 & $894 / 645$ & $120 / 56$ & GTR & 1.000 \\
TTR1 & $906 / 889$ & $151 / 73$ & TVM + G & 0.953 \\
vWF11 & $938 / 905$ & $181 / 98$ & HKY + G & 1.156 \\
autosomal loci & $5557 / 5139$ & $897 / 434$ & TVM + G & \\
combined & & & & 0.906 \\
Xq13.3 & $6634 / 4218$ & $621 / 327$ & TVM + G & 1.403 \\
DBY5 & $1048 / 662$ & $144 / 83$ & GTR + I & 1.500 \\
SMCY7 & $463 / 433$ & $89 / 56$ & TVM & 1.080 \\
SMCY11 & $606 / 541$ & $108 / 51$ & TVM & 0.948 \\
SRY & $786 / 772$ & $123 / 65$ & TVM & 1.082 \\
UTY18 & $881 / 828$ & $131 / 73$ & HKY + G & TVM \\
ZFYLI & $714 / 680$ & $106 / 54$ & TVM + G & \\
Y chromosomal loci & $4498 / 3916$ & $701 / 382$ & & 3.511 \\
combined & & & & \\
nuclear loci combined & $16689 / 13273$ & $2154 / 1143$ & TVM + G & \\
mitochondrial genome & $16860 / 15074^{*}$ & $6998 / 5457^{*}$ & GTR + I + G & \\
all data combined & $16860 / 11316^{* *}$ & $5745 / 4592^{* *}$ & GTR + I + G & \\
\hline
\end{tabular}

* mitochondrial alignment excluding indels, poorly aligned positions and D-loop

** mitochondrial alignment with only protein-coding genes 
Additional file 9: Additional Table 6. Primers and PCR conditions for the amplification of nuclear loci.

\begin{tabular}{lllll}
\hline Locus & Ref. & Forward primer & Reverse Primer & AT \\
\hline ALB3 & - & GCATTCAAAGTCAACCATG & ACGAAGAGTTGCAACTGTGC & $56^{\circ} \mathrm{C}$ \\
IRBP3 & - & CTCTGGACACACGCCCAG & CACACTGCTGGTCAGAATGA & $58^{\circ} \mathrm{C}$ \\
TNP2 & - & GCAGGTGTACAAAACCAAG & GTCTCATTAGTTGGATTTCC & $54^{\circ} \mathrm{C}$ \\
TTR1 & - & GGCCCTACGGTGAGTGTT & ACTTTGACCATCAGAGGACA & $56^{\circ} \mathrm{C}$ \\
vWF11 & {$[73]$} & see ref. & see ref. & see ref. \\
Xq13.3 & {$[10,75]$} & see ref. & see ref. & see ref. \\
DBY5 & {$[74]$} & see ref. & see ref. & see ref. \\
SMCY7 & {$[74]$} & see ref. & see ref. & see ref. \\
SMCY11 & {$[74]$} & see ref. & see ref. & see ref. \\
UTY18 & {$[74]$} & see ref. & see ref. & see ref. \\
SRY & {$[72]$} & see ref. & see ref. \\
ZFYLI & - & CCTGATTCCAGGCAGTACC & ATCAGGGCCAATAATTATTGCT & $58^{\circ} \mathrm{C}$ \\
\hline
\end{tabular}




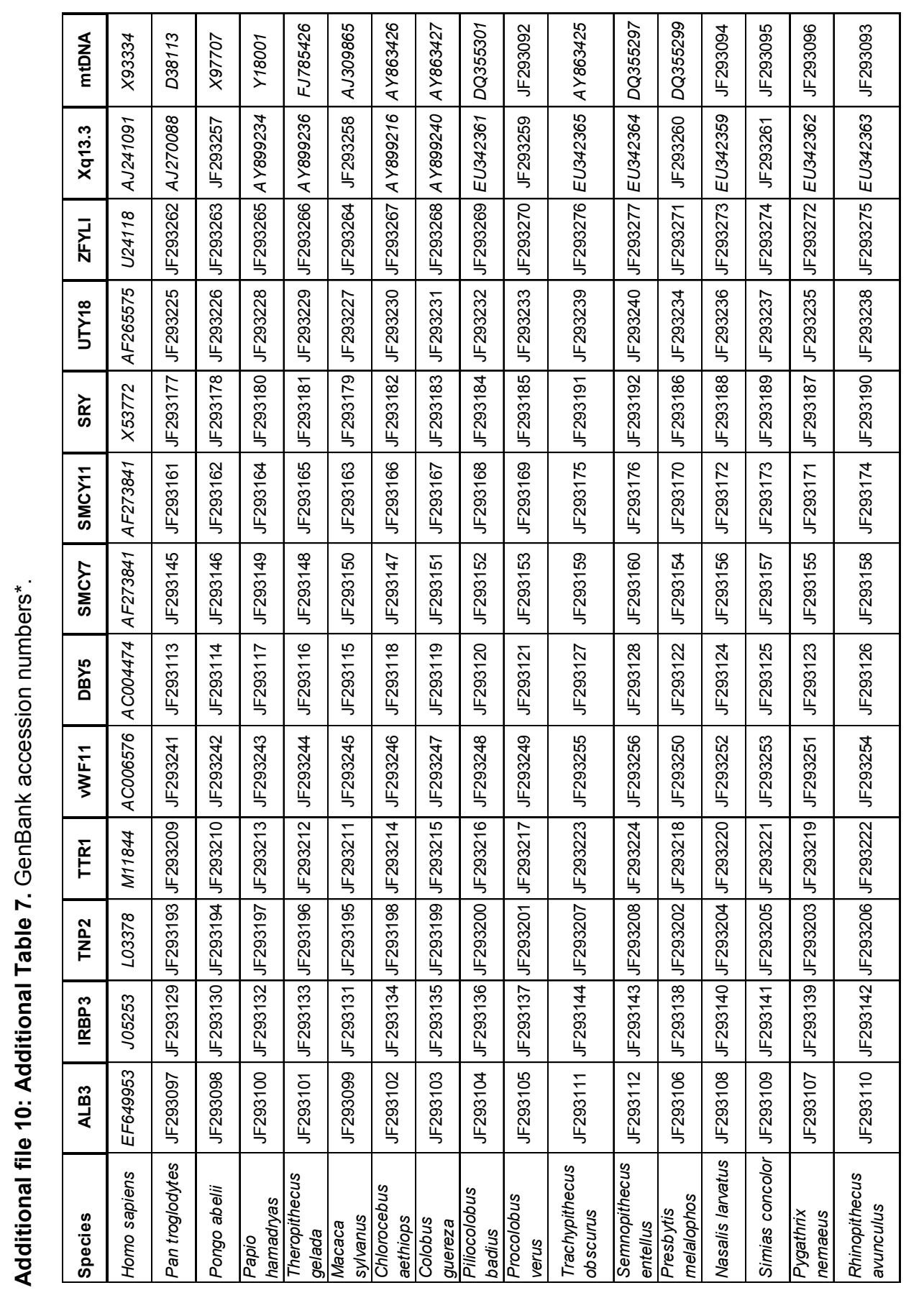


Chapter 2

\section{The Evolutionary History of Odd-Nosed Monkeys}

Published in:

Tan C, Grueter C, Wright B, editors.

Odd-nosed Monkeys: Recent Advances in the Study of the Forgotten Colobines, in press. 


\section{The Evolutionary History of Odd-Nosed Monkeys}

Mouyu Yang ${ }^{1,2}$, Tilo Nadler ${ }^{3}$, Dietmar Zinner ${ }^{4}$, Christian Roos ${ }^{5,6}$

${ }^{1}$ Primate Genetics Laboratory, German Primate Center, Göttingen, Germany

${ }^{2}$ Fanjingshan National Nature Reserve, Jiangkou, Guizhou Province, China

${ }^{3}$ Frankfurt Zoological Society, Endangered Primate Rescue Center, Cuc Phuong National Park, Ninh Binh Province, Vietnam

${ }^{4}$ Cognitive Ethology Laboratory, German Primate Center, Göttingen, Germany

${ }^{5}$ Gene Bank of Primates, German Primate Center, Göttingen, Germany

Corresponding author: Christian Roos, Gene Bank of Primates, Primate Genetics Laboratory, German Primate Center, D-37077 Göttingen, Germany; phone: +49 (0)551 3851300; croos@dpz.eu

Running title: Odd-nosed monkey evolution

Text pages (including acknowledgements and references): 17

Tables: 1

Figures: 2 


\section{Abstract}

The odd-nosed monkeys represent one of the two major groups among Asian colobines. Although a common origin of the group was recently confirmed, the phylogenetic relationships among its genera and species, and the patterns leading to their current distribution are largely unknown. To address these issues, we analyzed complete mitochondrial genome sequence data from nine of the ten odd-nosed monkey species, and reconstructed phylogenetic relationships and estimated divergence ages. Based on our data, we can confirm a common origin of Nasalis and Simias, but not of Rhinopithecus and Pygathrix. In fact, Rhinopithecus forms a sister clade to all other genera in the group, indicating a northern origin of odd-nosed monkeys and a later invasion into Indochina and Sundaland. According to our divergence age estimates, the genera Rhinopithecus, Pygathrix and Nasalis + Simias originated in the late Miocene, while speciation events within genera and also the split between Nasalis and Simias occurred in the Pleistocene.

\section{Keywords}

Odd-nosed monkey, phylogeny, evolution, biogeography 


\section{Introduction}

More than a decade ago, Nina Jablonski explained convincingly that odd-nosed monkeys represent a particular interesting group to study, and why they have been neglected scientifically for a long time (Jablonski 1998a). They are enigmatic and rare, and they show remarkable anatomical and behavioral adaptations to a range of, for primates, unusual habitats, such as mangrove swamps, and temperate and high altitude forests. The group consists of four genera, snub-nosed monkeys (Rhinopithecus), doucs langurs (Pygathrix), proboscis monkey (Nasalis) and pig-tailed monkey (Simias). Three of the five snub-nosed monkey species, $R$. roxellana, $R$. brelichi and $R$. bieti occur in a few isolated populations in China, while the fourth species, $R$. avunculus is restricted to the extreme North of Vietnam. A fifth species, $R$. strykeri was recently described from Myanmar (Geissmann et al. 2011). Douc langurs, represented by the three species $P$. nemaeus, $P$. cinerea and $P$. nigripes, are distributed through parts of Vietnam, Laos and Cambodia, east of the Mekong River. Both Nasalis and Simias are monotypic. N. lavatus is a pure Bornean species, whereas S. concolor is endemic to the Mentawai Islands, west of Sumatra. All species are endangered or even critically endangered (Geissmann et al. 2011; IUCN 2010). Since Jablonski's book, research has been intensified tremendously and a wealth of data was published concerning ecology, behavior and conservation of the Chinese species in particular (e.g. Grueter et al. 2009; Ren et al. 2008; Tan et al. 2007; Yang et al. 2009; Zhang et al. 2008).

Odd-nosed monkeys together with langurs, comprising the genera Presbytis, Semnopithecus and Trachypithecus, form the Asian colobines (Groves 2001; Jablonski 1998b; Jablonski and Peng 1993; Osterholz et al. 2008; Roos et al. 2011; Sterner et al. 2006; Ting et al. 2008). Although recent genetic investigations convincingly confirmed a common origin of the odd-nosed monkeys ( $\mathrm{Li}$ et al. 2011; Osterholz et al. 2008; Perelman et al. 2011; Roos et al. 2011; Sterner et al. 2006; Ting et al. 2008), the phylogenetic relationships among genera and species are still debated. Several phylogenetic hypotheses based on morphological or molecular arguments have been proposed including: (1) Rhinopithecus and Pygathrix are sister taxa (Delson 1975; Groves 2001; Jablonski and Peng 1993; Li et al. 2004; Napier 1985; Oates et al. 1994; Wang et al. 1997), (2) Pygathrix is more closely related to Nasalis than to Rhinopithecus (Jablonski 1998b), (3) Rhinopithecus and Nasalis are sister taxa (Zhang 
and Ryder 1998), (4) Simias is closely related to Nasalis (Whittaker et al. 2006), and (5) Simias is basal among Asian colobines (Jablonski 1998b).

In a study using complete mitochondrial genome sequences, Li et al. (2011) and Sterner et al. (2006) was not able to resolve relationships among the three studied genera Rhinopithecus, Pygathrix and Nasalis. Similar results were obtained from $\mathrm{X}$ chromosomal sequence data (Ting et al. 2008) and the presence/absence analysis of short interspersed elements (SINEs) (Osterholz et al. 2008). Unfortunately, none of these studies included Simias. However, based on a short fragment of the mitochondrial cytochrome b gene, Whittaker et al. (2006) suggested a sister grouping of Nasalis and Simias and a taxonomic placement of Simias within Nasalis. Latter arrangement was recently confirmed by a multi-maker approach using SINE integrations and sequence analysis of mitochondrial genomes as well as autosomal and gonosomal loci (Roos et al. 2011). Roos et al. (2011) also showed that Rhinopithecus is basal among odd-nosed monkeys.

Although relationships among odd-nosed monkey genera seem to be largely resolved now, this is not true for various relationships within them. Genetic data clearly resolve branching patterns within Pygathrix (Roos 2004; Roos and Nadler 2001; Roos et al. 2007), but relationships among the five Rhinopithecus species are still disputed. Jablonski and Peng (1993) found that $R$. roxellana grouped together with the $R$. bieti + $R$. brelichi clade and that $R$. avunculus was sister species to all other snub-nosed monkeys. Zhang and Ryder (1998), however, concluded that relationships between $R$. bieti, $R$. roxellana and $R$. avunculus are more or less three-way. Molecular studies by Roos (2004), Roos et al. (2007) and Li et al. (2004) support the view of Zhang and Ryder (1998). In a recent study by $\mathrm{Li}$ et al. (2011) using complete mitochondrial genome data, $R$. avunculus appeared as sister lineage to a $R$. bieti $+R$. roxellana clade. Unfortunately, $R$. strykeri was not included in all these studies, but the species resembles $R$. bieti in various characters (Geissmann et al. 2011).

Although our understanding of the phylogenetic relationships within the oddnosed monkey group became clearer due to molecular studies, several questions remain. However, all molecular studies so far used only short mitochondrial fragments and/or did not include all species. To obtain a more complete picture about the evolutionary history of the odd-nosed monkeys, we have analyzed the complete mitochondrial genome from nine of the ten odd-nosed monkey species. Assuming that 
the origin of the odd-nosed monkey group was in the North of its current range (Hengduan Mountains and southern Yunnan, Jablonski 1998b; Roos et al. 2011), we suppose a north-south migration of the group with corresponding successive divergences of the respective more southern taxa. As a result of our study, we present here the most complete and updated molecular phylogeny of odd-nosed monkeys and discuss their phylogeographic implications.

\section{Methods}

Sample Collection

Fecal samples from each one individual of $R$. roxellana, R. brelichi, R. bieti, $P$. nigripes and $P$. cinerea were obtained from the breeding station of Fanjingshan National Nature Reserve, China, Beijing Zoo, China, and the Endangered Primate Rescue Center, Vietnam, respectively. Fresh samples were preserved in tubes with $80 \%$ ethanol. Mitochondrial genome sequences from $R$. avunculus, $P$. nemaeus, $N$. larvatus and S. concolor were recently published by our group (Roos et al. 2011).

\section{Laboratory Methods}

DNA from fecal material was extracted using the Qiagen Stool Mini kit following recommendations of the supplier. To reduce the likelihood of amplifying nuclear pseudogenes (numts), complete mitochondrial genomes were amplified via five overlapping fragments, each with a size of $\sim 5,000 \mathrm{bp}$. All these long-range PCRs were performed with the SuperTaq Plus polymerase from Ambion following protocols of the supplier and primers as described (Raaum et al. 2005; Roos et al. 2011; Sterner et al. 2006). Long-range PCR amplicons were separated on $1 \%$ agarose gels, excised from the gel, purified with the Qiagen Gel Extraction kit and used as template for nested PCRs. PCR conditions for all nested PCR amplifications were identical and comprised a pre-denaturation step at $94^{\circ} \mathrm{C}$ for $2 \mathrm{~min}$, followed by 30 cycles each with denaturation at $94^{\circ} \mathrm{C}$ for $1 \mathrm{~min}$, annealing at $60^{\circ} \mathrm{C}$ for $1 \mathrm{~min}$, and extension at $72^{\circ} \mathrm{C}$ for $1.5 \mathrm{~min}$. At the end, a final extension step at $72^{\circ} \mathrm{C}$ for 5 min was added. Nested PCR products (900 - 1,200 bp in length) were cleaned with the Qiagen PCR Purification kit and sequenced on an $\mathrm{ABI} 3130 \mathrm{xl}$ sequencer. Sequences were assembled with Geneious v4.6.1 (Drummond et al. 2009). No inconsistent positions in overlapping regions were detected and all protein-coding genes were correctly translated. Annotation of 
mitochondrial genomes was conducted with the online program DOGMA (Wyman et al. 2004) and manually inspected.

\section{Statistical Analysis}

For phylogenetic reconstructions, we implemented further mitochondrial genome sequences deposited in GenBank. The final dataset comprised 22 sequences including each one representative of the nine studied odd-nosed monkey species and Presbytis melalophos, Semnopithecus entellus, Trachypithecus obscurus, Colobus guereza, Piliocolobus badius, Procolobus verus, four cercopithecine species (Papio hamadryas, Theropithecus gelada, Macaca sylvanus, Chlorocebus aethiops), and three hominoid species (Homo sapiens, Pan troglodytes, Pongo abelii), which were used as outgroup taxa. The alignment was generated with MAFFT v6 (Katoh et al. 2005) and corrected by eye. Poorly aligned positions and indels were removed with Gblocks v0.91b (Castresana 2000) using default settings.

Phylogenetic trees were constructed with maximum-likelihood (ML) and Bayesian algorithms, using the programs GARLI v0.951 (Zwickl 2006) and MrBayes v3.1.2 (Huelsenbeck et al. 2001; Ronquist and Huelsenbeck 2003). For both reconstructions, the GTR + I + G model was applied as it was chosen as optimal nucleotide substitution model using the Bayesian information criterion (BIC) as implemented in jMODELTEST v0.1 (Posada 2008). Relative support of internal nodes for $M L$ reconstructions was performed by bootstrap analyses with 500 replications. In GARLI, only the model specification settings were adjusted, while all other settings were left at their default value. ML majority-rule consensus trees were calculated in PAUP v4.0b10 (Swofford 2003). For Bayesian reconstructions, we used four Monte Carlo Markov Chains (MCMC) with the default temperature of 0.1 . Four repetitions were run for 10,000,000 generations with tree and parameter sampling occurring every 100 generations. The first $25 \%$ of samples were discarded as burnin, leaving 75,001 trees per run. Posterior probabilities for each split and a phylogram with mean branch lengths were calculated from the posterior density of trees.

To estimate divergence ages, a Bayesian Markov Chain Monte Carlo (MCMC) method, which employs a relaxed molecular clock approach (Drummond et al. 2006), as implemented in BEAST v1.4.8 (Drummond and Rambaut 2007), was used. A 
relaxed lognormal model of lineage variation and a Birth-Death Process prior for branching rates was assumed.

As calibrations we used the fossil-based divergence between Homo and Pan, which has been dated at 6 - 7 million years ago (mya) (Brunet et al. 2005; Lebartard et al. 2008; Vignaud et al. 2002), the separation of Pongo from the Homo + Pan lineage 14 mya (Kelley 2002), the split between Theropithecus and Papio 4 mya (Leakey 1993; Delson 2000), and the divergence of hominoids and cercopithecoids 23 mya (Benefit and McCrossin 2002; Young and MacLatchy 2004; Zalmout et al. 2010). Instead of hardbounded calibration points, we used the published dates as a normal distribution prior for the respective node. For the Homo - Pan divergence, this translates into a normal distribution with a mean of 6.5 mya and a standard deviation (SD) of 0.5 mya, for the separation of Pongo from the Homo + Pan clade into a mean of 14.0 mya and a SD of 1.0 mya, for the Theropithecus - Papio split into a mean of 4.0 mya and a SD of 0.5 mya, and for the hominoid - cercopithecoid divergence into a mean of 23 mya and a SD of 2 mya.

Since the estimation of phylogenetic relationships was not the main aim of this analysis, we used an a-priori fixed tree topology as obtained from tree reconstructions mentioned above. Four replicates were run for 10,000,000 generations with tree and parameter sampling occurring every 100 generations. The adequacy of a $10 \%$ burnin and convergence of all parameters were assessed by visual inspection of the trace of the parameters across generations using TRACER v1.4.1 (Rambaut and Drummond 2007). Subsequently, the sampling distributions were combined ( $25 \%$ burnin) using the software LogCombiner v1.4.8 and a consensus chronogram with node height distribution was generated and visualized with TreeAnnotator v1.4.8 and FigTree v1.2.2 (Rambaut 2008).

\section{Results}

We successfully generated complete mitochondrial genome sequences from $R$. roxellana, $R$. brelichi, $R$. bieti, $P$. nigripes and $P$. cinerea. The complete alignment including nine of the ten odd-nosed monkey species and various other catarrhine taxa had a length of $16,910 \mathrm{bp}$. After the removal of indels and poorly aligned positions, the alignment was $15,608 \mathrm{bp}$ in length. Among them, 7,352 sites were variable and 5,924 of them parsimony-informative. 


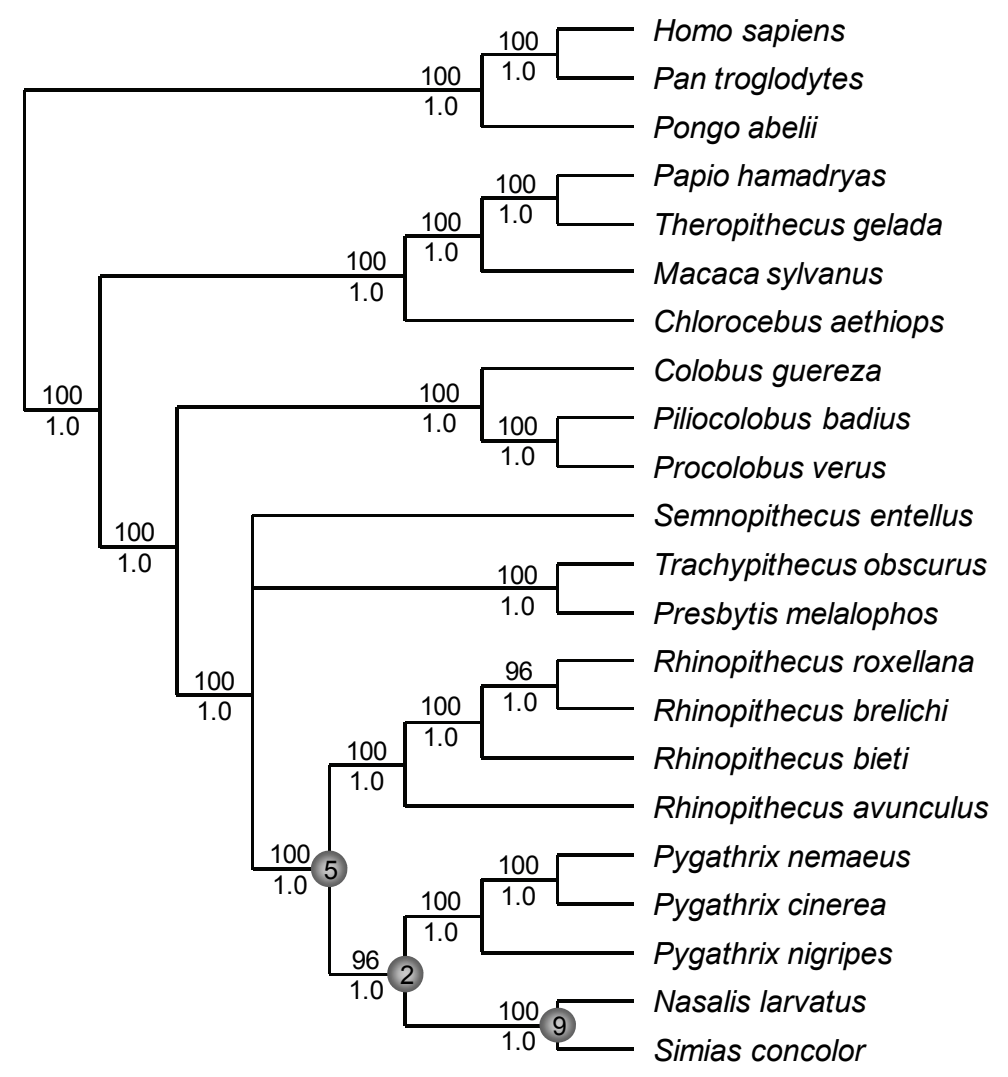

Fig. 1: Phylogenetic relationships among 22 catarrhine primates including nine of the ten odd-nosed monkey species. Numbers on branches indicate support values as obtained from ML and Bayesian reconstructions. Light grey circles and impeded numbers refer to number of SINE integrations supporting respective nodes (SINE data from Roos et al. 2011).

Phylogenetic reconstructions based on $\mathrm{ML}$ and Bayesian algorithms revealed identical tree topologies. With the exception of the unresolved relationship among the odd-nosed monkey clade, the Trachypithecus + Presbytis clade and Semnopithecus, all other branching patterns were well resolved and significantly supported (Fig. 1). According to our reconstructions and divergence age estimations (Fig. 2, Table 1), the initial split occurred between hominoids and cercopithecoids 23.99 mya (for 95\% credibility intervals $[\mathrm{Cl}$ s see Table 1). Among the former, Pongo branched off 13.44 mya, followed by the divergence of Pan and Homo 6.16 mya. 16.84 mya, cercopithecoids further segregated into two reciprocally monophyletic clades referring to Cercopithecinae and Colobinae. In the former, Chlorocebus represented the most basal split (10.91 mya), while Macaca was sister lineage to the Papio + Theropithecus clade. Macaca diverged from Papio + Theropithecus 9.11 mya and latter two separated 
4.03 mya. Colobine monkeys further segregated into an African and an Asian clade 10.85 mya. Among African colobines, Colobus branched off first (8.48 mya), before finally also Piliocolobus and Procolobus separated (6.72 mya). Asian colobines initially diverged 8.60 mya into an odd-nosed monkey clade, a Trachypithecus + Presbytis clade and Semnopithecus, but the relationships among these three lineages remained unresolved. Within odd-nosed monkeys, Rhinopithecus was most basal and Pygathrix formed a sister lineage to the Nasalis + Simias clade. The last common ancestors of the odd-nosed monkeys and of the Pygathrix + Nasalis + Simias clade occurred 6.85 and 6.30 mya, respectively. Nasalis and Simias finally differentiated 1.98 mya. In Rhinopithecus, $R$. avunculus diverged first (2.38 mya), followed by R. bieti (1.93 mya), before finally also $R$. brelichi and $R$. roxellana separated (1.66 mya). Within Pygathrix, $P$. nigripes was basal to the $P$. cinerea $+P$. nemaeus clade. According to our estimates, the split between $P$. nigripes and the other two species occurred 2.51 mya, while latter two separated 0.69 mya.

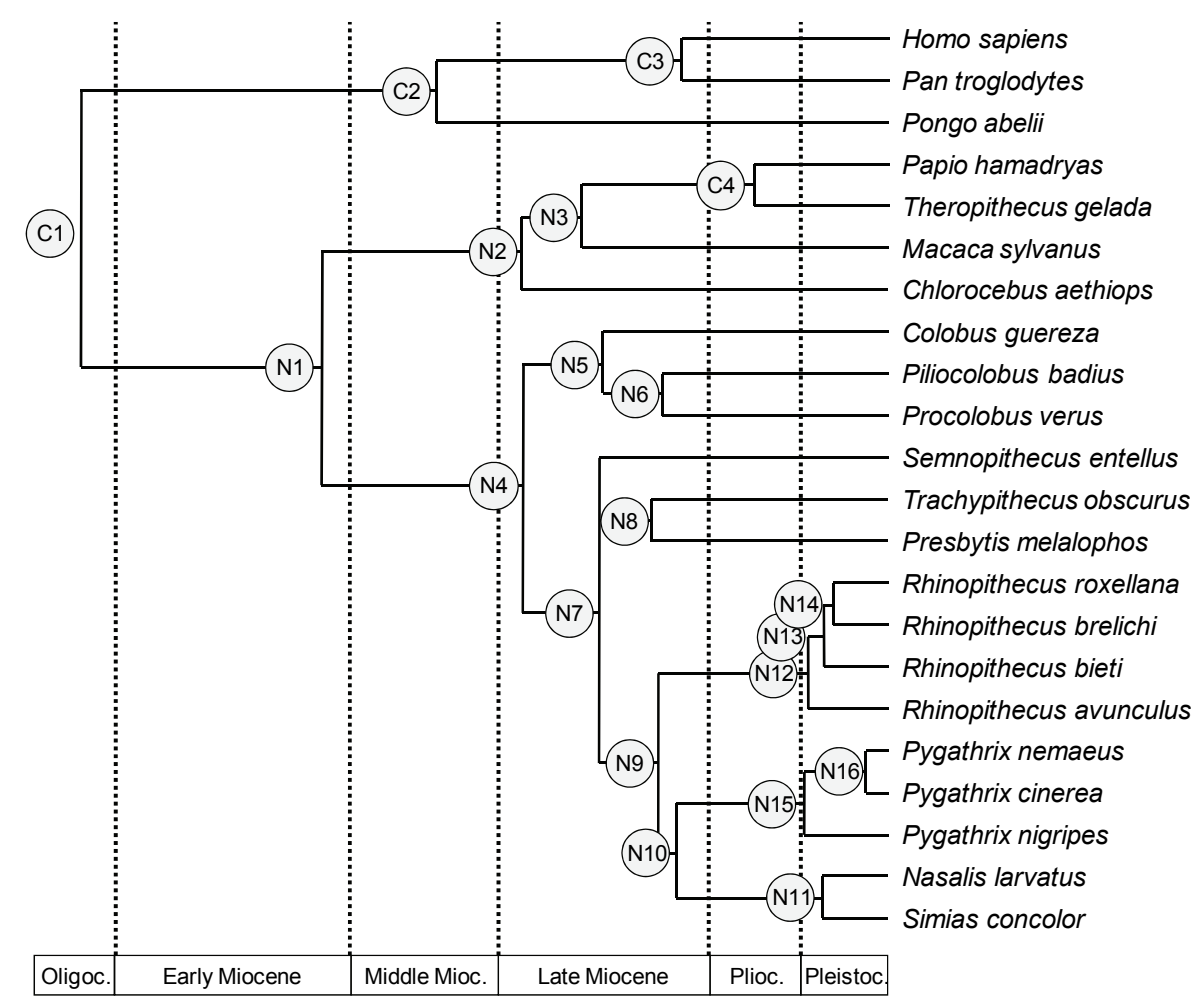

Fig. 2: Ultrametric tree showing phylogenetic relationships and estimated divergence ages among 22 catarrhine primates including nine of ten odd-nosed monkey species. Nodes labeled with $\mathrm{C}$ and $\mathrm{N}$ refer to fossil-based calibration points and nodes of interest, respectively. For full details of estimated divergence ages see Table 1. 
Table 1: Divergence age estimations in mya (see also Fig. 2)

\begin{tabular}{|l|l|c|}
\hline Node & Divergence & Mean (95\% CI) \\
\hline C1 & Cercopithecoidea - Hominoidea & $23.99(22.18-26.10)$ \\
\hline C2 & Pongo - (Homo + Pan) & $13.44(12.43-14.56)$ \\
\hline C4 & Homo - Pan & $6.16(5.62-6.72)$ \\
\hline N1 & Cercopithecinae - Colobinae & $4.03(3.52-4.57)$ \\
\hline N2 & Chlorocebus - (Macaca + Papio + Theropithecus) & $16.84(14.59-19.15)$ \\
\hline N3 & Macaca - (Papio + Theropithecus) & $10.91(9.08-12.66)$ \\
\hline N4 & African Colobinae - Asian Colobinae & $9.11(7.41-10.78)$ \\
\hline N5 & Colobus - (Piliocolobus + Procolobus) & $10.85(9.65-12.08)$ \\
\hline N6 & Piliocolobus - Procolobus & $8.48(7.09-9.82)$ \\
\hline N7 & odd-nosed monkeys - Semnopithecus - (Trachypithecus + & $6.72(5.27-8.05)$ \\
\hline N8 & Presbytis) & $8.60(7.77-9.43)$ \\
\hline N9 & Rhinopithecus - (Pygathrix + Nasalis + Simias) & $7.05(5.96-8.02)$ \\
\hline N10 & Pygathrix - (Nasalis + Simias) & $6.85(5.94-7.69)$ \\
\hline N11 & Nasalis - Simias & $6.30(5.43-7.16)$ \\
\hline N12 & R. avunculus - (R. bieti + R. brelichi + R. roxellana) & $1.98(1.36-2.56)$ \\
\hline N13 & R. bieti - (R. brelichi + R. roxellana) & $2.38(1.91-2.85)$ \\
\hline N14 & R. brelichi - R. roxellana & $1.93(1.51-2.37)$ \\
\hline N15 & P. nigripes - (P. nemaeus + P. cinerea) & $1.66(1.24-2.09)$ \\
\hline N16 & P. nemaeus - P. cinerea & $2.51(1.87-3.15)$ \\
\hline
\end{tabular}

\section{Discussion}

As in earlier molecular studies (Osterholz et al. 2008; Perelman et al. 2011; Roos et al. 2011; Sterner et al. 2006; Ting et al. 2008), the monophyly of the oddnosed monkeys is strongly supported and further underpinned by five SINE integrations (Roos et al. 2011). Further, our data clearly suggest a basal position of Rhinopithecus and a sister grouping of Pygathrix and Nasalis + Simias, a pattern, which is also supported by two SINE integrations (Roos et al. 2011) and nuclear sequence data (Perelman et al. 2011; Roos et al. 2011). Moreover, our study provides clear information concerning the branching pattern within the two polytypic genera Rhinopithecus and Pygathrix. Since the overall relationships and estimated divergence ages among various lineages are in agreement with earlier studies (Goodman et al. 1998; Karanth et al. 2008; Osterholz et al. 2008; Perelman et al. 2011; Raaum et al. 
2005; Roos et al. 2011; Sterner et al. 2006; Ting 2008; Ting et al. 2008; Xing et al. 2005), our data can be regarded as true and reliable.

\section{Taxonomy of odd-nosed monkeys}

Our study corroborates a three genera and ten species classification of the oddnosed monkey group. Although $R$. strykeri was not studied, it is provisionally classified as distinct species here. All three clades, representing the three genera, diverged from a common ancestor in the latest Miocene. The divergence of Simias and Nasalis occurred in the early Pleistocene, a time frame in which most species of Rhinopithecus and Pygathrix emerged. Only the separation between $P$. nemaeus and $P$. cinerea occurred later, about 0.69 mya. Therefore, our data does not support the perpetuation of Simias as a genus or subgenus, but suggests its inclusion in Nasalis. Accordingly, the genus Nasalis contains two species, N. larvatus and N. concolor. Further, our data does not support the placement of $R$. avunculus in the subgenus Presbyticus, because the divergence time between $R$. avunculus and the other snub-nosed monkey species was estimated at roughly the same time as other species' splits in Rhinopithecus and Pygathrix. To avoid the erection of a subgenus for $P$. nigripes, we prefer the five species ( $R$. avunculus, $R$. bieti, $R$. brelichi, $R$. roxellana, $R$. strykeri) classification of Rhinopithecus and the three species classification of Pygathrix $(P$. cinerea, $P$. nemaeus, $P$. nigripes). Although $P$. cinerea and $P$. nemaeus diverged relative recently, both split in the same range as various other Asian colobines (Roos, unpublished), and, thus, species level for both is proposed.

\section{Evolutionary history and biogeography of odd-nosed monkeys}

By combining the available information, we develop the following dispersal scenario for odd-nosed monkeys. The origin of Asian colobine monkeys and also of the odd-nosed monkeys might have been the Hengduan Mountains in the border region of today's Burma, India and China (Jablonski 1998b; Peng et al. 1993; Roos et al. 2011). In the region, all the larger Southeast Asian rivers (Mekong, Salween, Yangtze) rise, which are all well known as barriers for arboreal primates (Meijaard and Groves 2006) and which exist at least since the early Miocene (Hallet and Molnar 2001). After the separation of the langur progenitor from the odd-nosed monkey ancestor, members of the odd-nosed monkey group successively migrated from China to the south and 
expanded their range into Indochina and Sundaland in the latest Miocene. The speciation within Rhinopithecus occurred in the early Pleistocene, most likely triggered by reduction and fragmentation of suitable habitat. For Pygathrix, our data suggest an origin in southern Vietnam and Cambodia. From there, the ancestor of $P$. nemaeus and $P$. cinerea migrated to the north in the early to middle Pleistocene. The separation of latter two occurred on a similar time scale as species splits among crested gibbons (Thinh et al. 2010) and limestone langurs (Perelman et al. 2011), suggesting tremendous environmental changes during that time. The migration of a Nasalis + Simias progenitor into Sundaland was probably via land bridges connecting the mainland with Sundaland islands during periods of lowered sea levels (Miller et al. 2005). Finally, Nasalis on Borneo and Simias on the Mentawai islands diverged in the early Pliocene. Most likely, Simias or at least a progenitor of Simias and Nasalis was also present on Sumatra but went extinct there. Due to the dating discrepancy (mitochondrial data: 1.98 mya, nuclear data: 1.1 mya, Roos et al. 2011), further gene flow between both genera after the initial separation until the end of the early Pleistocene cannot be excluded, especially considering that migration was repeatedly possible via land bridge connections during the Pleistocene (Miller et al. 2005).

\section{Conclusions}

By analyzing complete mitochondrial genome sequence data from nine of the ten odd-nosed monkey species, the present study provides comprehensive insight into the evolutionary and biogeographic history of this enigmatic primate group. Most importantly, our study shows that odd-nosed monkeys originated on the Asian mainland and migrated into Indochina and Sundaland during the late Miocene. Differentiation into species occurred during the Pleistocene. Although our study is the first, which allows such detailed information about phylogeny and phylogeography of odd-nosed monkeys, future studies should also include the newly described $R$. strykeri as well as nuclear sequence data which might allow to uncover possible ancient hybridization events as it was detected in the case of the Asian colobine genus Trachypithecus (Roos et al. 2011). 


\section{References}

Benefit BR, McCrossin KL (2002) Old World monkeys: the Miocene emergence. In: Hartwig W (ed) The primate fossil record. Cambridge University Press, Cambridge

Brunet M, Guy F, Pilbeam D et al (2005) New material of the earliest hominid from the Upper Miocene of Chad. Nature 434:752-755

Castresana J (2000) Selection of conserved blocks from multiple alignments for their use in phylogenetic analysis. Mol Biol Evol 17:540-552

Delson E (1975) Evolutionary history of the Cercopithecidae. Contrib Primatol 5:167-217

Delson E (2000) Cercopithecinae. In: Delson E, Tattersall I, Van Couvering JA, Brooks AS (eds) Encyclopedia of human evolution and prehistory. Garland Publishing Inc, New York

Drummond AJ, Rambaut A (2007) BEAST: Bayesian evolutionary analysis by sampling trees. BMC Evol Biol 7:214

Drummond AJ, Ho SYN, Phillips MJ et al (2006) Relaxed phylogenetics and dating with confidence. PLoS Biol 4:e88

Drummond AJ, Ashton B, Cheung M et al (2009) Geneious v4.6. Available from http://www.geneious.com/

Geissmann T, Lwin N, Aung SS et al (2011) A new species of snub-nosed monkey, genus Rhinopithecus Milne-Edwards, 1872 (Primates, Colobinae), from northern Kachin state, northeastern Myanmar. Am J Primatol 73:96-107

Goodman M, Porter CA, Czelusniak J et al (1998) Toward a phylogenetic classification of primates based on DNA evidence complemented by fossil evidence. Mol Phylogenet Evol 9:585-598

Groves CP (2001) Primate taxonomy. Smithsonian Institution Press, Washington, DC

Grueter CC, Li D, Ren B et al (2009) Dietary profile of Rhinopithecus bieti and its socioecological implications. Int J Primatol 30:601-624

Hallet B, Molnar P (2001) Distorted drainage basins as markers of crustal strain east of the Himalayas. J Geophys Res 106:13697-13709

Huelsenbeck JP, Ronquist F, Nielsen R et al (2001) Bayesian inference of phylogeny and its impact on evolutionary biology. Science 294:2310-2314 
IUCN (2010) IUCN red list of threatened species, Version 2010.1. www.iucnredlist.org. Accessed 9 June 2010

Jablonski NG (1998a) The natural history of the doucs and snub-nosed monkeys. World Scientific, Singapore

Jablonski NG (1998b) The evolution of the doucs and snub-nosed monkey and the question of the phyletic unity of the odd-nosed colobines. In: Jablonski NG (ed) The natural history of the doucs and snub-nosed monkeys. World Scientific, Singapore

Jablonski NG, Peng YZ (1993) The phylogenetic relationships and classification of the doucs and snub-nosed langurs of China and Vietnam. Folia Primatol 60:36-55

Karanth KP, Singh L, Collura RV et al (2008) Molecular phylogeny and biogeography of langurs and leaf monkeys of South Asia (Primates: Colobinae). Mol Phylogenet Evol 46:683-694

Katoh K, Kuma K, Toh $\mathrm{H}$ et al (2005) MAFFT version 5: improvement in accuracy of multiple sequence alignment. Nucleic Acids Res 33:511-518

Kelley J (2002) The hominoid radiation in Asia. In: Hartwig WC (ed) The primate fossil record. Cambridge University Press, Cambridge

Leakey MG (1993) Evolution of Theropithecus in the Turkana basin. In: Jablonski NG (ed) Theropithecus, the rise and fall of a primate genus. Cambridge University Press, Cambridge

Lebatard AE, Bourlès DL, Duringer $P$ et al (2008) Cosmogenic nuclide dating of Sahelanthropus tchadensis and Australopithecus bahrelghazali: Mio-Pliocene hominids from Chad. Proc Natl Acad Sci USA 105:3226-3231

Li M, Wei F, Huang C et al (2004) Phylogeny of snub-nosed monkeys inferred from mitochondrial DNA, cytochrome $b$, and $12 \mathrm{~S}$ rRNA sequences. Int J Primatol 25:861-873

Li Y, Wang X, Ting N, Zhang YP (2011) Mitogenomic analysis of Chinese snubnosed monkeys: Evidence of positive selection in NADH dehydrogenase genes in highaltitude adaptation. Mitochondrion 11:497-503

Meijaard E, Groves CP (2006) The geography of mammals and rivers in mainland South-East Asia. In: Lehman SM, Fleagle, JG (eds) Primate biogeography. Springer, New York

Miller KG, Kominz MA, Browning JV et al (2005) The phanerozoic record of global sea-level change. Science 310:1293-1298 
Napier PH (1985) Catalogue of primates in the British Museum (Natural History) and elsewhere in the British Isles. Part III: Family Cercopithecidae, subfamily Colobinae. London, British Museum (Natural History)

Oates JF, Davies AG, Delson E (1994) The diversity of living colobines. In: Davies AG, Oates JF (eds) Colobine monkeys: their ecology, behaviour and evolution. Cambridge University Press, Cambridge

Osterholz M, Walter L, Roos C (2008) Phylogenetic position of the langur genera Semnopithecus and Trachypithecus among Asian colobines, and genus affiliations of their species groups. BMC Evol Biol 8:58

Peng YZ, Pan RL, Jablonski N (1993) Classification and evolution of Asian colobines. Folia Primatol 60:106-117

Perelman P, Johnson WE, Roos C et al (2011) Am molecular phylogeny of living primates. PLoS Genet 7:e1001342

Posada D (2008) jModelTest: Phylogenetic model averaging. Mol Biol Evol 25:1253-1256

Raaum RL, Sterner KN, Noviello CM et al (2005) Catarrhine primate divergence dates estimated from complete mitochondrial genomes: concordance with fossil and nuclear DNA evidence. J Hum Evol 48:237-257

Rambaut A (2008) FigTree: Tree figure drawing tool, version 1.2.2. Institute of Evolutionary Biology, University of Edinburgh. http://tree.bio.ed.ac.uk/software/figtree/

Rambaut A, Drummond AJ (2007) Tracer v1.4.1: MCMC trace analysis tool. Institute of Evolutionary Biology, University of Edinburgh. http://tree.bio.ed.ac.uk/software/tracer/

Ren B, Li M, Long $Y$ et al (2008) Measuring daily ranging distances of Rhinopithecus bieti via a Global Positioning System collar at Jinsichang, China: a methodological consideration. Int J Primatol 29:783-794

Ronquist F, Huelsenbeck JP (2003) MrBayes 3: Bayesian phylogenetic inference under mixed models. Bioinformatics 19:1572-1574

Roos C (2004) Molecular evolution and systematics of Vietnamese primates. In: Nadler T, Streicher U, Long HT (eds) Conservation of primates in Vietnam. Frankfurt Zoological Society, Frankfurt

Roos C, Nadler T (2001) Molecular evolution of the douc langurs. Zool Garten NF 71:1-6 
Roos C, Thanh VN, Walter L et al (2007) Molecular systematics of Indochinese primates. Vietn J Primatol 1:41-53

Roos C, Zinner D, Kubatko LS et al (2011) Nuclear versus mitochondrial DNA: Evidence for hybridization in colobine monkeys. BMC Evol Biol 11:77

Sterner KN, Raaum RL, Zhang YP etal (2006) Mitochondrial data support an odd-nosed colobine clade. Mol Phylogenet Evol 40:1-7

Swofford DL (2003) PAUP*: Phylogenetic analysis using parsimony (*and other methods), version 4. Sinauer, Sunderland

Tan C, Guo S, Li B (2007) Population structure and ranging patterns of Rhinopithecus roxellana in Zhouzhi National Nature Reserve, Shaanxi, China. Int J Primatol 28:577-591

Thinh, VN, Mootnick A, Geissmann T et al (2010) Mitochondrial evidence for multiple radiations in the evolutionary history of small apes. BMC Evol Biol 10:74

Ting N (2008) Mitochondrial relationships and divergence dates of the African colobines: evidence of Miocene origins for the living colobus monkeys. J Hum Evol 55:312-325

Ting N, Tosi AJ, Li Y et al (2008) Phylogenetic incongruence between nuclear and mitochondrial markers in the Asian colobines and the evolution of the langurs and leaf monkeys. Mol Phylogenet Evol 46:466-474

Vignaud P, Duringer P, Mackaye HT et al (2002) Geology and palaeontology of the Upper Miocene Toros-Menalla hominid locality, Chad. Nature 418:152-155

Wang W, Forstner MRJ, Zhang YP et al (1997) A phylogeny of Chinese leaf monkeys using mitochondrial ND3-ND4 gene sequences. Int J Primatol 18:305-320

Whittaker DJ, Ting N, Melnick DJ (2006) Molecular phylogenetic affinities of the simakobu monkey (Simias concolor). Mol Phylogenet Evol 39:887-892

Wyman SK, Jansen RK, Boore JL (2004) Automatic annotation of organellar genomes with DOGMA. Bioinformatics 20:3252-3255

Xing J, Wang H, Han K et al (2005) A mobile element based phylogeny of Old World monkeys. Mol Phylogenet Evol 37:872-880

Yang M, Sun DY, Zinner D et al (2009) Reproductive parameters in Guizhou snub-nosed monkeys (Rhinopithecus brelichi). Am J Primatol 71:266-270

Young NM, MacLatchy L (2004) The phylogenetic position of Moropithecus. J Hum Evol 46:163-184 
Zalmout IS, Sanders WJ, MacLatchy LM et al (2010) New Oligocene primate fossil from Saudi Arabia and the divergence of apes and Old World monkeys. Nature 466:360-365

Zhang P, Watanabe K, Li B (2008) Female social dynamics in a provisioned free-ranging band of the Sichuan snub-nosed monkey (Rhinopithecus roxellana) in the Qinling mountains, China. Am J Primatol 70:1013-1022

Zhang YP, Ryder OA (1998) Mitochondrial cytochrome b gene sequences of Old World monkeys: with special reference on evolution of Asian colobines. Primates 39:39-49

Zwickl DJ (2006) Genetic algorithm approaches for the phylogenetic analysis of large biological sequence data sets under the maximum likelihood criterion. Ph.D. Dissertation. University of Texas, Austin 


\title{
Chapter 3
}

\author{
Population Genetic Structure of \\ Guizhou Snub-Nosed Monkeys (Rhinopithecus brelichi) \\ as Inferred from Mitochondrial Control Region Sequences, \\ and Comparison with $R$. roxellana and $R$. bieti
}

Published in:

AMERICAN JOURNAL OF PHYSICAL ANTHROPOLOGY,

Doi 10.1002/ajpa.21618. 


\title{
Population Genetic Structure of Guizhou Snub-Nosed Monkeys (Rhinopithecus brelichi) as Inferred From Mitochondrial Control Region Sequences, and Comparison With $R$. roxellana and $R$. bieti
}

\author{
Mouyu Yang, ${ }^{1,2 \star}$ Yeqin Yang, ${ }^{2}$ Duoying Cui, ${ }^{3}$ Gisela Fickenscher, ${ }^{4}$ \\ Dietmar Zinner, ${ }^{4}$ Christian Roos, ${ }^{1,5}$ and Markus Brameier ${ }^{1}$ \\ ${ }^{1}$ Primate Genetics Laboratory, German Primate Center, Göttingen 37077, Germany \\ ${ }^{2}$ Fanjingshan National Nature Reserve, Jiangkou 554400, Guizhou Province, China \\ ${ }^{3}$ Beijing Zoo, Beijing 100044, China \\ ${ }^{4}$ Cognitive Ethology Laboratory, German Primate Center, Göttingen 37077, Germany \\ ${ }^{5}$ Gene Bank of Primates, German Primate Center, Göttingen 37077, Germany
}

\section{KEY WORDS hypervariable region I; phylogeny; evolution; Rhinopithecus; conservation}

\begin{abstract}
The Guizhou snub-nosed monkey (Rhinopithecus brelichi) is a primate species endemic to the Wuling Mountains in southern China. With a maximum of 800 wild animals, the species is endangered and one of the rarest Chinese primates. To assess the genetic diversity within $R$. brelichi and to analyze its genetic population structure, we collected fecal samples from the wild $R$. brelichi population and sequenced the hypervariable region $\mathrm{I}$ of the mitochondrial control region from 141 individuals. We compared our data with those from the two other Chinese snub-nosed species ( $R$. roxellana, $R$. bieti) and reconstructed their phylogenetic relationships and divergence times. With only five haplotypes and a maximum of 25 polymorphic sites, $R$. brelichi shows the lowest genetic diversity in terms of haplotype diversity $(h)$, nucleotide diversity $(\pi)$, and average number of pairwise nucleotide differences (П). The most
\end{abstract}

recent common ancestor of $R$. brelichi lived $\sim 0.36$ million years ago $(\mathrm{Ma})$, thus more recently than those of $R$. roxellana $(\sim 0.91 \mathrm{Ma})$ and $R$. bieti $(\sim 1.33 \mathrm{Ma})$. Phylogenetic analysis and analysis of molecular variance revealed a clear and significant differentiation among the three Chinese snub-nosed monkey species. Population genetic analyses (Tajima's D, Fu's $\mathrm{F}_{\mathrm{s}}$, and mismatch distribution) suggest a stable population size for $R$. breli$c h i$. For the other two species, results point in the same direction, but population substructure possibly introduces some ambiguity. Because of the lower genetic variation, the smaller population size and the more restricted distribution, $R$. brelichi might be more vulnerable to environmental changes or climate oscillations than the other two Chinese snub-nosed monkey species. Am J Phys Anthropol 000:000-000, 2011. @2011 Wiley-Liss, Inc.

Quang, Bac Kan, Thai Nguyen, and Ha Giang provinces of northern Vietnam, and R. strykeri in Kachin state of Burma (Groves, 2001; International Union for Conservation of Nature (IUCN), 2010; Geissmann et al., 2011). Among the five species, $R$. roxellana with $\sim 15,000$ individuals has the largest population (IUCN, 2010), followed by $R$. bieti with a maximum of 2,000 individuals (IUCN, 2010) and $R$. brelichi with 750-800 individuals (Yang et al., 2002; IUCN, 2010). For $R$. avunculus and $R$. strykeri, with only 200 and 260-330 individuals, respectively, the situation is even worse (Mittermeier et al., 2009; IUCN, 2010; Geissmann et al., 2011). Accord-

Grant sponsor: Beijing Zoo and the German Primate Center.

\footnotetext{
*Correspondence to: Mouyu Yang, Primate Genetics Laboratory, German Primate Center, Kellnerweg 4, Göttingen 37077, Germany. E-mail: myang@dpz.eu
}

Received 5 September 2010; accepted 10 August 2011

DOI 10.1002/ajpa.21618

Published online in Wiley Online Library (wileyonlinelibrary.com). 


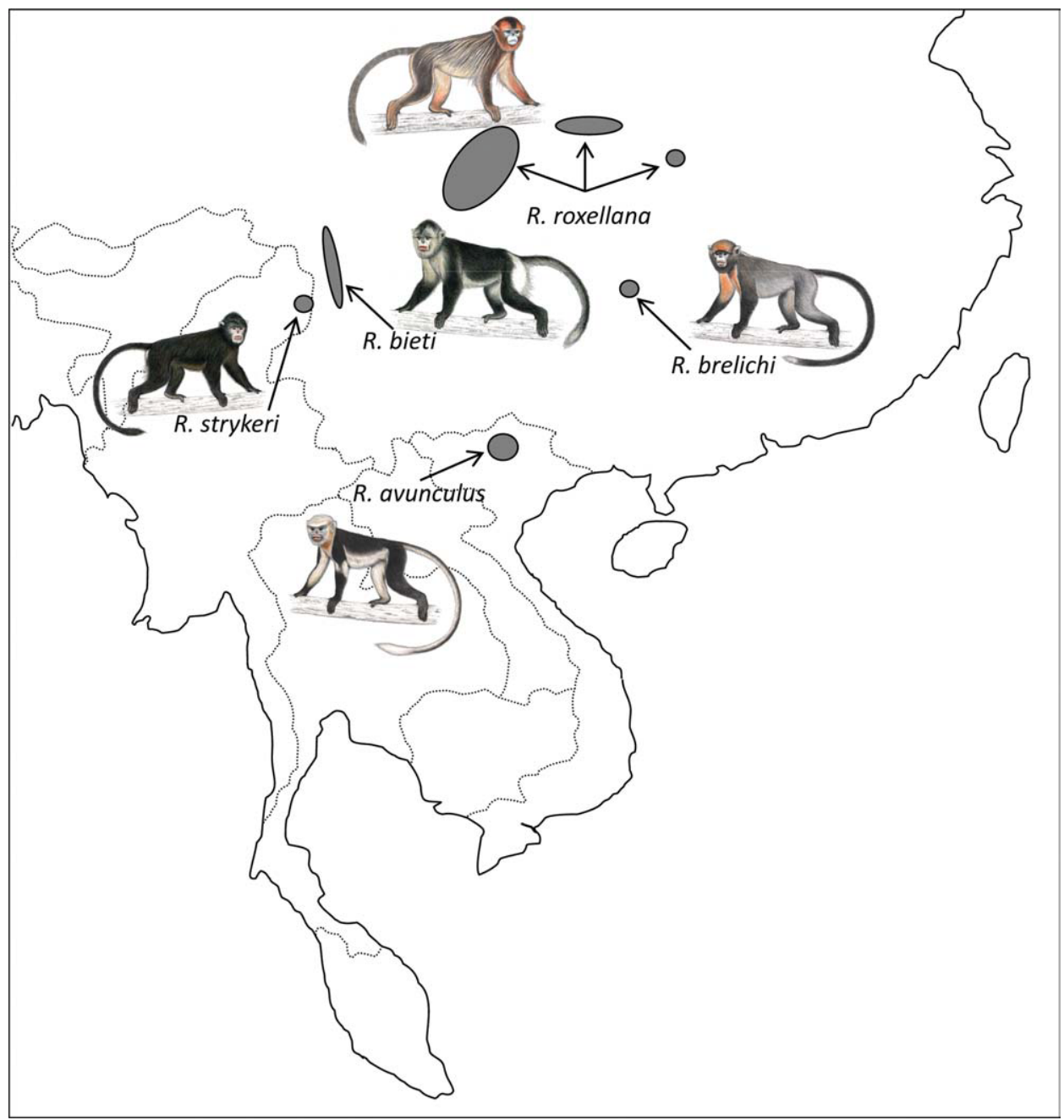

Fig. 1. Schematic distribution of the five snub-nosed monkey species. Dotted lines indicate country borders. Illustrations by Stephen Nash, Conservation International. [Color figure can be viewed in the online issue, which is available at wileyonlinelibrary. com.]

ingly, the three Chinese species are classified as "Endangered" and $R$. avunculus as "Critically Endangered" (IUCN, 2010). R. strykeri is yet unclassified, but most likely deserves the status of a "Critically Endangered" species as well (Geissmann et al., 2011).

Of the Chinese snub-nosed monkey species, $R$. brelichi is the least studied and it exhibits the most restricted distribution. It occurs only in evergreen and deciduous broadleaf forests at altitudes of $1,400-2,300 \mathrm{~m}$ in the Fanjingshan National Nature Reserve (FNNR) in the Wuling Mountains, Guizhou province (Groves, 2001; Wu et al., 2004; MacKinnon, 2008; IUCN, 2010). Within the FNNR, the species inhabits a core area of about $260 \mathrm{~km}^{2}$ (Bleisch and Xie, 1998; Yang et al., 2002). Thus, among the three Chinese species, $R$. brelichi is the only one, which comprises only one continuous population (Yang et al., 2002), while $R$. bieti consists of various geographically distinct subpopulations (Liu et al., 2007) and $R$. roxellana even of three subspecies (Li et al., 2007; IUCN, 2010). Furthermore, compared to its Chinese congenerics, $R$. brelichi shows a longer interbirth interval (3 years compared to 2 years in $R$. roxellana and $R$. bieti; Ji et al., 1998; Zhang et al., 2000; Cui et al., 2006; Qi et al., 2008; Yang et al., 2009) and a later age at first reproduction in females (8-9 years compared to 5-6 years in $R$. roxellana and $R$. bieti; Ji et al., 1998; Liang et al., 2001; Qi et al., 2008; Yang et al., 2009). Accordingly, among the three Chinese species, $R$. brelichi might hold the lowest population recovery potential.

Various studies on the phylogeny and phylogeography within the genus Rhinopithecus exist (Zhang and Ryder, 1998; Li et al., 2004; Roos et al., 2007; Yang et al., in press), but population genetic data are available only for $R$. bieti and $R$. roxellana ( $\mathrm{Li}$ et al., 2001; Li et al., 2003; $\mathrm{Li}$ et al., 2007; Liu et al., 2007). As genetic diversity within a species is regarded, beside other factors such as population size, reproductive output, environmental changes, and human activities, as an important factor for the long-term survival of species, population genetic analyses contribute also to the assessment of a species' conservation status (Dietz et al., 2000; Di Fiore, 2003; Spielman et al., 2004; Hoeglund, 2009).

In our study, we aimed to analyze and compare the genetic variation within and among the three Chinese snub-nosed species, their genetic population structure and their evolutionary history. Therefore, we sequenced the hypervariable region I (HVI) of the mitochondrial control region from $\sim 20 \%$ of the total $R$. brelichi population and compared them to orthologous sequences of $R$. roxellana and $R$. bieti available from GenBank and the 
literature ( $\mathrm{Li}$ et al., 2007; Liu et al., 2007). As R. brelichi has the smallest population size and the most restricted distribution of the Chinese species, we expect a lower genetic diversity than reported from the other two species (hypothesis 1), and a population size reduction during its demographic history (hypothesis 2).

\section{MATERIALS AND METHODS Sample collection}

In September/October 2007 and 2008, 146 fecal samples from $R$. brelichi individuals were collected in Yangaoping, an area of about $77 \mathrm{~km}^{2}$ in the north of FNNR (N $127^{\circ} 58^{\prime}$; E $108^{\circ} 45^{\prime}$ ). In this region, roughly 600 individuals and thus the majority of the $R$. brelichi population come together twice a year (March-April, September-October). Accordingly, a division of the species into subpopulations is not indicated (Yang et al., 2002).

The animals were observed, and the samples were collected immediately after the monkeys left. To minimize the probability of collecting several samples from the same individuals, only samples with a distance of at least $2 \mathrm{~m}$ from each other were taken. Samples were preserved in plastic tubes with $70 \%$ ethanol and stored at room temperature before further processing.

Our study complied with protocols approved by the Forestry Ministry of Guizhou Province, China, and adhered to the legal requirements of the countries in which research was conducted. The study was carried out in compliance with respective animal care regulations and the principles of the American Society of Primatologists and the German Primate Center for the ethical treatment of nonhuman primates.

\section{Individual identification}

DNA from fecal samples was extracted using the QIAamp DNA Stool Mini Kit (Qiagen, Germany). From each sample, $\sim 200 \mathrm{mg}$ of feces was taken, pounded, and incubated overnight on a rotating wheel in argininosuccinate lyase (ASL) buffer. Extraction was further performed following the instructions of the manufacturer with the exception that, in the last step, the DNA was diluted in $100 \mu \mathrm{l}$ of high-performance liquid chromatography (HPLC) water instead of tris chloride elution buffer (AE) buffer. Extracted DNA was stored at $-20^{\circ} \mathrm{C}$ before further processing. For each sample, two independent extractions were done.

To exclude the possibility that more than one sample per individual was included in the analysis, all samples were genotyped at eight polymorphic microsatellite loci (D1S533, D2S1326, D6S264, D6S501, D7S2204, D8S505, D10S1432, and D17S1290). Because of the low amount of DNA extracted from fecal samples, allelic dropout and null alleles tend to produce incorrect genotypes (Morin et al., 2001). Thus, we repeated genotyping multiple times depending on the DNA concentration of the sample following the approach of Morin et al. (2001). Accordingly, genotyping was repeated at least three times for heterozygous, and depending on the DNA amount three to seven times for homozygous samples.

No indications for null alleles, large allelic dropout or scoring errors due to stuttering were detected (MicroChecker 2.2.3; van Oosterhout et al., 2004). The probability of identity $P_{(\mathrm{ID})}$ (Waits et al., 2001), i.e., the probability that two individuals drawn at random from a population have the same genotype at multiple loci, was calculated with GenAlex 6.4 (Peakall and Smouse, 2006). In our estimation, $P_{(\mathrm{ID})}$ was $6.9 \times 10^{-6}$ over eight microsatellite loci. In wildlife forensic identification, $P_{(\text {ID) }}$ $<0.001-0.0001$ is required (Waits et al., 2001). On the basis of the direct observations and the calculation of the probability of identity, we were able to identify 141 individuals from 146 fecal samples.

\section{Mitochondrial DNA amplification and sequencing}

From the 141 individually assigned samples, we polymerase chain reaction (PCR)-amplified $\sim 650 \mathrm{bp}$ of the HVI using the primers 5'-AAATGAACCTGCCCTTGTAGT-3' and 5'-GAGGATAGA ACCAGATGTCC-3'. PCR conditions consisted of a predenaturation step at $94^{\circ} \mathrm{C}$ for $5 \mathrm{~min}$, followed by 40 cycles, each with a denaturation step at $94^{\circ} \mathrm{C}$ for $1 \mathrm{~min}$, annealing at $60^{\circ} \mathrm{C}$ for $1 \mathrm{~min}$, and extension at $72^{\circ} \mathrm{C}$ for $1 \mathrm{~min}$. At the end, a final extension step at $72^{\circ} \mathrm{C}$ for $5 \mathrm{~min}$ was added. The results of the PCR amplifications were checked on 1\% agarose gels. Negative controls (reactions without DNA template) were used to check PCR performance and contamination. Subsequently, PCR products were cleaned with the Qiagen PCR Purification Kit and sequenced on an ABI PRISM 3130xL Genetic Analyzer (Applied Biosystems, Germany) with the BigDye Cycle Sequencing Kit (Applied Biosystems, Germany). To further exclude contamination, we randomly repeated amplification and sequencing from the second DNA extract for 40 samples. Haplotype sequences were deposited to GenBank (HQ891105-HQ891109).

\section{Statistical methods: Interspecies analysis}

Sequences with a length of $603 \mathrm{bp}$ were manually verified and aligned in BioEdit (Hall, 1999). For comparative purposes, orthologous sequences of $R$. roxellana (60 individuals) and $R$. bieti (157 individuals), published in earlier studies (Li et al., 2007; Liu et al., 2007), were taken from GenBank. Because of length differences, we trimmed the alignment to a length of $379 \mathrm{bp}$ (positions 125-504 of the original $R$. brelichi alignment). Analysis of molecular variance (AMOVA) (Excoffier et al., 1992) was performed to examine the genetic differentiation and relationship among $R$. brelichi, $R$. roxellana and $R$. bieti populations with 10,000 permutations as implemented in Arlequin 3.5.1.2 (Excoffier and Lischer, 2010). Genetic relationships were estimated by comparing the average number of pairwise nucleotide differences between populations from two different species $\left(\pi_{x y}\right)$, the average number of pairwise differences within each species $\left(\pi_{x}\right)$, and the corrected average pairwise difference $\left(\pi_{x y}-\left(\pi_{x}+\pi_{y}\right) / 2\right)$. The genetic distance among species was determined by using the population's pairwise phiCT indices and $P$ value (Wright, 1969, 1978). As a general rule, phiCT values above 0.25 indicate high genetic differentiation between populations (Nei 1977; Wright, 1978; de Jong et al., 1994; Hartl and Clark, 2007).

A median-joining network (Bandelt et al., 1999) was created with Network 4.6 to visualize the topology of the population structure based on frequencies and phylogenetic relationships among HVI haplotypes both at the intraspecific and interspecific level.

Phylogenetic relationships among haplotypes were constructed with maximum-likelihood (ML) and Bayesian algorithms, using the programs Garli 0.951 (Zwickl, 2006) and MrBayes 3.1.2 (Huelsenbeck et al., 2001; Ron- 
quist and Huelsenbeck, 2003). Therefore, the orthologous sequence from $R$. avunculus (EU004480, Osterholz et al., 2008) was taken from GenBank and used as an outgroup. For both reconstructions, the HKY $+\mathrm{G}$ model was used as it was chosen as best-fit model by the Bayesian Information Criterion in jModeltest 0.1 (Posada, 2008). In Garli, only the model specification settings were adjusted according to the dataset, while all other settings were left at their default values. Relative support of internal nodes was assessed by bootstrap analyses with 500 replications. A majority-rule consensus tree was calculated in PAUP* 4.0b10 (Swofford, 2003). For Bayesian reconstructions, four Markov Chain Monte Carlo (MCMC) runs with the default temperature of 0.1 were used. Four repetitions were run for 10,000,000 generations with tree and parameter sampling occurring every 100 generations. The first $25 \%$ of samples were discarded as burnin, leaving 75,001 trees per run. Posterior probabilities for each split and a phylogram with mean branch lengths were calculated from the posterior density of trees.

A Bayesian MCMC method, which uses a relaxed molecular clock approach (Drummond et al., 2006), as implemented in BEAST 1.4.8 (Drummond and Rambaut, 2007), was used to estimate divergence times. Therefore, a relaxed lognormal model of lineage variation and a Birth-Death prior for branching rates was assumed. As no appropriate fossils are available as calibration points, we used a mean HVI substitution rate of 0.1643 substitutions per nucleotide per million years (Myr) (Soares et al., 2009). The 95\% confidence interval for the normal distribution of substitution rates ranged from 0.06 to 0.25 substitutions per site per Myr (Santos et al., 2005). We analyzed four replicates for 25,000,000 generations with tree and parameter sampling occurring every 100 generations. The adequacy of a $10 \%$ burnin and convergence of all parameters were assessed by visual inspection of the trace of the parameters across generations using Tracer 1.4.1 (Rambaut and Drummond, 2007). Subsequently, the sampling distributions were combined (25\% burnin) using the software LogCombiner 1.4.8 and a consensus chronogram with node height distribution was generated and visualized with TreeAnnotator 1.4.8 and FigTree 1.2.2 (Rambaut, 2008).

\section{Statistical methods: Intraspecies analysis}

Genetic diversity within species was estimated by using haplotype diversity $(h)$, nucleotide diversity $(\pi)$ (Nei and Li, 1979; Nei, 1987), and the average number of pairwise nucleotide differences (П) (Wakeley, 1997) in Arlequin 3.5 (Excoffier and Lischer, 2010). To support hypothesis 1 of low genetic diversity in $R$. brelichi, all these values should be lower in this species than in the two congenerics.

The haplotype network furthermore allows us to investigate whether any genetic population structure exists in $R$. brelichi. Within $R$. roxellana and $R$. bieti geographic substructures have been identified and have been shown to correspond partly to genetic substructures ( $\mathrm{Li}$ et al., 2007; Liu et al., 2007). In R. brelichi, however, no geographically identifiable subpopulations exist (Yang et al., 2002). Therefore, we expect no comparable substructures in the current $R$. brelichi population, which would be reflected by distinct clusters in the median-joining network. The mutational distance between different haplotypes in the network is therefore expected to be clearly smaller within $R$. brelichi than within the other two species. Population structure would be additionally supported by lower $\Pi$ values. Note that $\Pi$ equals the withinpopulation parameter of AMOVA $\left(\pi_{x}\right)$ and thus is a direct measure of the genetic variation within a population.

To explore the demographic history of the populations, we calculated Tajima's D statistic (Tajima, 1989) and Fu's $\mathrm{F}_{\mathrm{S}}$ test $(\mathrm{Fu}, 1997)$ with 10,000 permutations as implemented in Arlequin 3.5. These statistics are applied to infer the population growth history under the null hypothesis of selective neutrality and population equilibrium. A significant Tajima's D value or Fu's $F_{S}$ value indicates departure from the null hypothesis. In addition, we use the mismatch distribution analysis in Arlequin and DnaSP 5.10.01 (Librado and Rozas, 2009) using a demographic expansion model as a baseline. The sum of squared differences (SSD; Schneider and Excoffier, 1999) and the raggedness index $(r)$ (Harpending, 1994) are indicators for how far the observed distribution deviates from the expected smooth and unimodal distribution of the model.

To support our second hypothesis, a reduction of population size in $R$. brelichi, we should find (1) significantly positive Tajima's $\mathrm{D}$ and Fu's $\mathrm{F}_{\mathrm{s}}$ values, indicating a wider spectrum of allele frequencies in the population, and (2) an unimodal shape of the mismatch distribution with low parameter values SSD and $r$, indicating no (significant) deviation from the underlying model of expansion (or decline).

In contrast, both, nonsignificant $\mathrm{D}$ and $\mathrm{F}_{\mathrm{s}}$ values implying no deviation from the neutral model, and a multimodal (or "ragged") shape of the mismatch distribution (Rogers and Harpending, 1992) with significant and high-index values, would indicate for populations that remained largely stable in size during their history.

It should be noted here that none of these statistics for investigating the demographic history of a population is very reliable by itself and may depend on additional factors. For instance, a positive Tajima's D may also be the result of other processes that promote maintaining many alleles not only in a population, in particular balancing selection, but also in population subdivision and migration (Schmidt and Pool, 2002). Only the combination of different indicators, if ever, can make reliable predictions about the demographic history.

\section{RESULTS}

We successfully PCR-amplified and sequenced the HVI from $141 R$. brelichi samples, which were confirmed to originate from different individuals by microsatellite analysis. All amplifications produced a single band with a size of $\sim 650 \mathrm{bp}$. Randomly repeated amplification and sequencing for 40 samples revealed identical sequences. Of the $603 \mathrm{bp}, 26$ sites were variable, including one transversion (tv) and 25 transitions (ts). Among the 141 individuals, we observed five haplotypes (see Table 1). To compare the population genetic parameters among $R$. brelichi, $R$. roxellana and $R$. bieti, we trimmed the alignment to a length of $379 \mathrm{bp}$. By doing so, only one variable site in the $R$. brelichi sequence (position 33 of the original 603 bp-alignment) was excluded.

\section{Differentiation among the three Chinese species of Rhinopithecus}

AMOVA results reveal a significant genetic differentiation among the three species (Table 2). The average 
TABLE 2. Genetic differentiation analysis (AMOVA) between $R$. brelichi, $R$. roxellana, and $R$. bieti ${ }^{a}$

\begin{tabular}{lccc}
\hline & $\begin{array}{c}R . \text { brelichi } \\
\text { and } R \text {. roxellana }\end{array}$ & $\begin{array}{c}R . \text { brelichi } \\
\text { and } R \text {. bieti }\end{array}$ & $\begin{array}{c}R \text {. roxellana } \\
\text { and } R \text {. biet } i\end{array}$ \\
\hline$\pi_{x y}$ & 40.8559 & 51.006 & 51.8529 \\
$\pi_{x y}-\left(\pi_{x}+\pi_{y}\right) / 2$ & 31.4625 & 41.4895 & 39.208 \\
phiCT $(P$ value $)$ & $0.803(<0.001)$ & $0.810(<0.001)$ & $0.741(<0.001)$
\end{tabular}

${ }^{\mathrm{a}} \pi_{x y}$, average number of pairwise nucleotide differences between populations; $\pi_{x y}-\left(\pi_{x}+\pi_{y}\right) / 2$, corrected average pairwise difference; $\pi_{x}$, within-population values $\left(\pi_{x}=\Pi\right.$ in Table 3$)$; phiCT, pairwise measure of genetic differentiation between populations.

number of pairwise nucleotide differences between populations $\left(\pi_{x y}\right)$ results in the following estimates: $R$. brelichi and $R$. roxellana $\pi_{x y}=40.9 ; R$. brelichi and $R$. bieti $\pi_{x y}$ $=51.0 ; R$. roxellana and $R$. bieti $\pi_{x y}=51.9$. The corrected average pairwise difference $\left(\pi_{x y}-\left(\pi_{x}+\pi_{y}\right) / 2\right)$ amounts to 31.5 for $R$. brelichi and $R$. roxellana, 41.5 for $R$. brelichi and $R$. bieti, and 39.2 for $R$. roxellana and $R$. bieti. Values for $R$. brelichi and $R$. bieti, and for $R$. roxellana and $R$. bieti are similar, but higher than the value for $R$. brelichi and $R$. roxellana. According to the population-specific phiCT, a large proportion of the genetic variation is triggered by disparities between the species $(R$. brelichi and $R$. roxellana: phiCT $=0.803, R$. brelichi and $R$. bieti: phiCT $=0.810, R$. roxellana and $R$. bieti: phiCT $=0.741$; Table 2$)$. All phiCT values are statistically significant $(P<0.001)$ indicating that all three species are distinct from each other. The lower phiCT value between $R$. roxellana and $R$. bieti is most likely triggered by their higher intraspecific diversity.

The topology of the median-joining network (Fig. 2) confirms a clear genetic differentiation between the three species by showing significantly larger haplotype distances between than within each species. The network corroborates that despite a similar number of individual samples, the diversity of haplotypes is much lower within $R$. brelichi than within $R$. bieti and $R$. roxellana. In $R$. bieti and $R$. roxellana, a division into the two described major clades, A and B, (Li et al., 2007; Liu et al., 2007) can be discerned. In contrast, there are no apparent substructures in $R$. brelichi.

The phylogenetic tree reconstructions based on $\mathrm{ML}$ and Bayesian algorithms resulted in almost identical tree topologies, but the relationships among the haplotypes within major clades differed slightly (Fig. 3). Accordingly, the three Chinese species form strongly supported monophyletic clades and, although only weakly supported, $R$. roxellana and $R$. brelichi are suggested as sister taxa. Based on divergence age estimations, $R$. avunculus separated from the Chinese species 2.34 (1.66-3.05) million years ago (Ma), and $R$. bieti from $R$. roxellana and $R$. brelichi $2.19(1.62-2.80)$ Ma, before finally latter two diverged 1.77 (1.17-2.36) Ma. The most recent common ancestors (MRCA) of $R$. bieti and $R$. roxellana lived $1.33(0.82-1.90)$ and $0.91(0.53-1.31) \mathrm{Ma}$, while the MRCA of $R$. brelichi has a similar age $(0.36$ [0.17-0.56] Ma) as clades $\mathrm{A}$ and $\mathrm{B}$ of $R$. bieti (A: 0.24 [0.11-0.37] Ma, B: 0.10 [0.03-0.19] $\mathrm{Ma}$ ) and $R$. roxellana (A: 0.46 [0.21-0.66] Ma, B: 0.36 [0.17-0.56] Ma).

\section{Intraspecific variation}

Haplotype diversity $(h)$ and nucleotide diversity $(\pi)$ for the three Chinese snub-nosed monkey species are sum- 
Page 66

YANG ET AL.

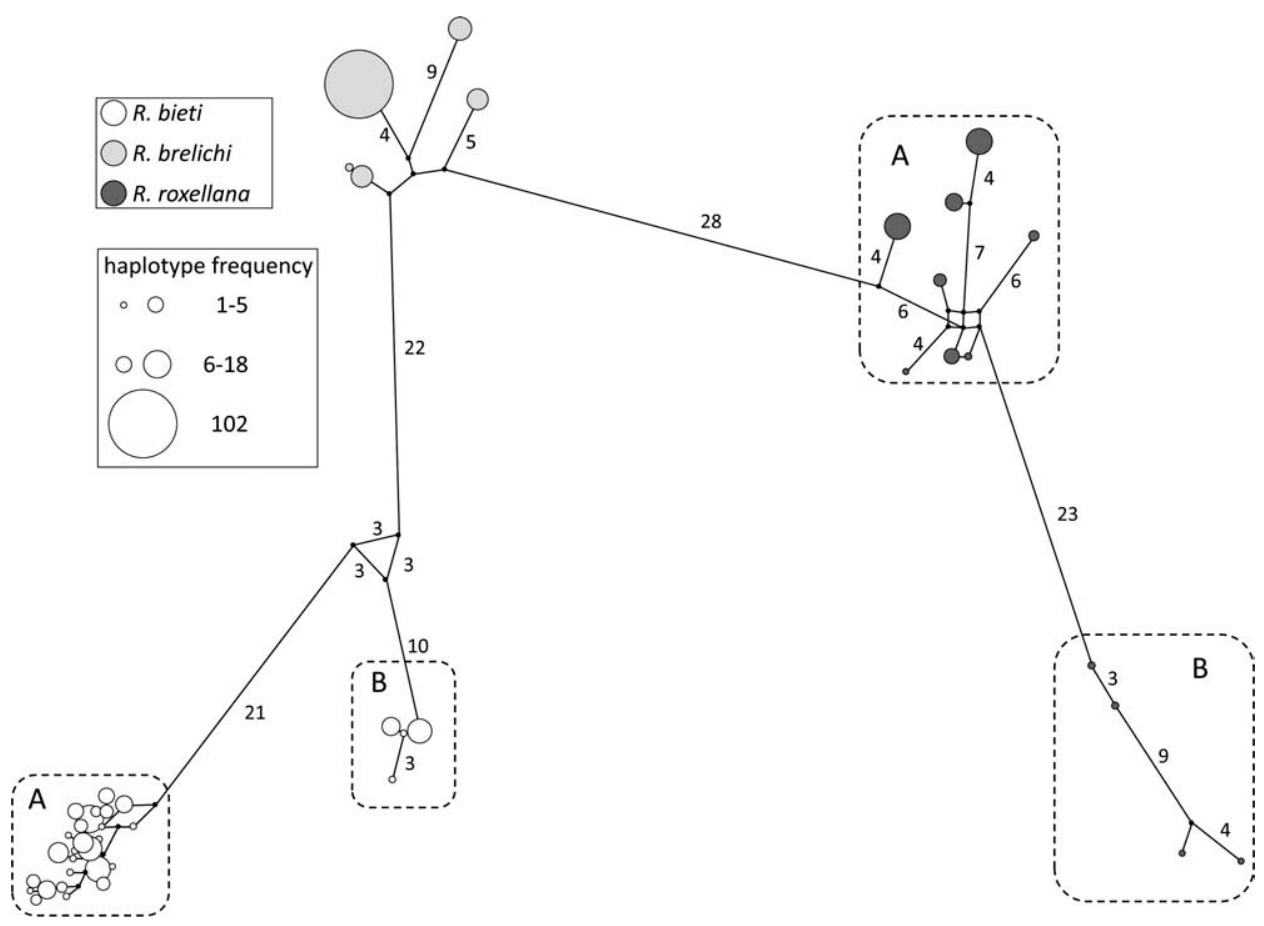

Fig. 2. Median-joining network of HVI haplotypes of the three Chinese Rhinopithecus species. Haplotype frequencies are reflected by node sizes. Links are proportional to number of mutational steps (also indicated by numbers for links with more than two mutational steps). A and $\mathbf{B}$ refer to respective subclades of $R$. roxellana and $R$. bieti.

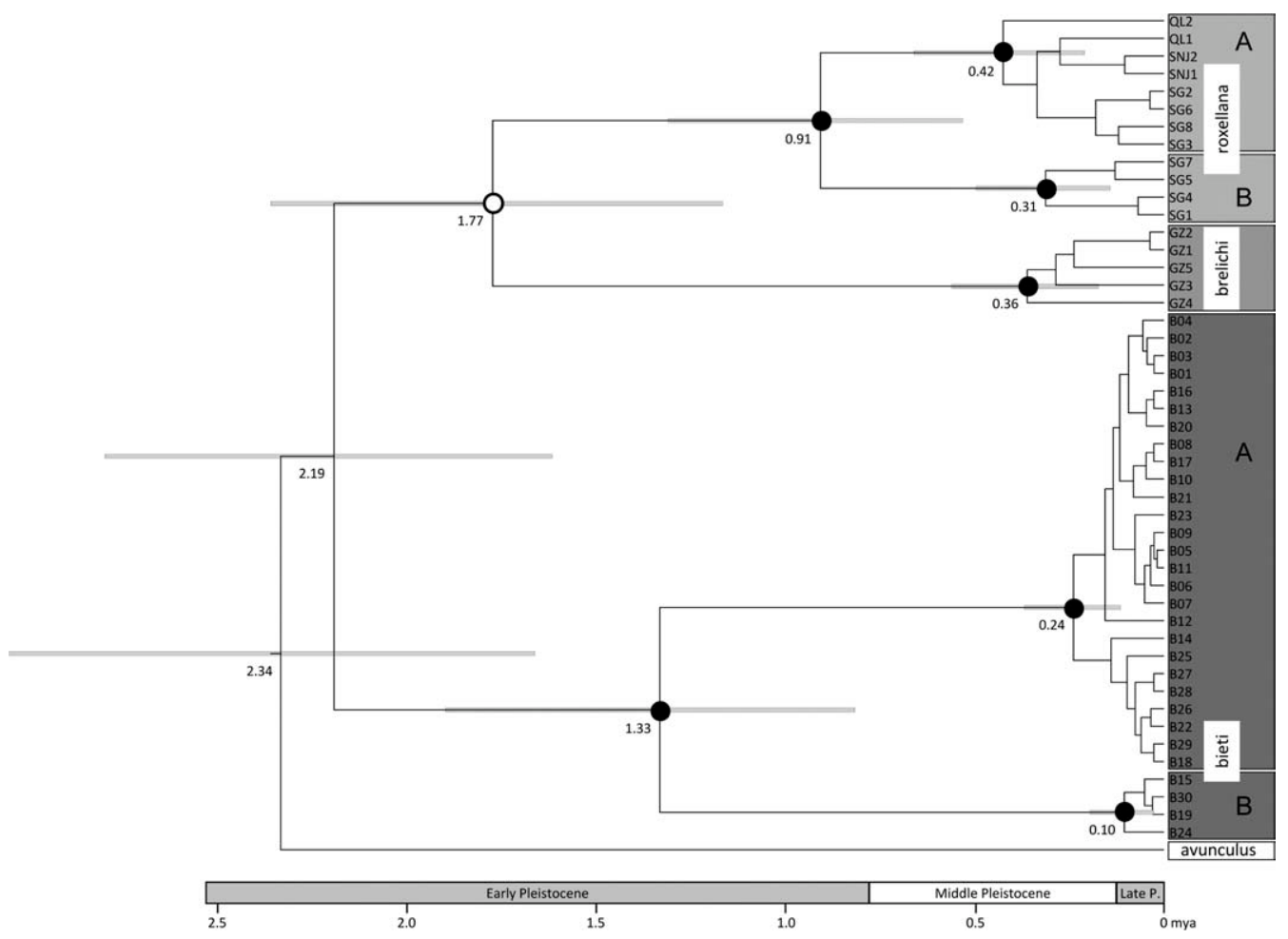

Fig. 3. Ultrametric tree showing phylogenetic relationships and estimated divergence ages among studied Rhinopithecus haplotypes. $\mathbf{A}$ and $\mathbf{B}$ refer to respective subclades in $R$. roxellana and $R$. bieti. Numbers on nodes indicate mean divergence ages and bars respective $95 \%$ highest posterior densities. Support values for ML and Bayesian reconstructions are indicated as circles (black circles: $\geq 97 \%, 1.0$; open circle: $<50 \%, 0.92$ ). 
TABLE 3. Population genetic variables for $R$. brelichi, $R$. roxellana, and $R$. bieti

\begin{tabular}{|c|c|c|c|}
\hline & R. brelichi & R. roxellana & R. bieti \\
\hline No. of individuals & 141 & 60 & 157 \\
\hline No. of nucleotide sites & 379 & 379 & 379 \\
\hline No. of polymorphic sites $(S)$ & 25 & 54 & 51 \\
\hline No. of haplotypes & 5 & 12 & 30 \\
\hline Haplotype diversity $(h)$ & $0.457 \pm 0.048$ & $0.845 \pm 0.026$ & $0.945 \pm 0.006$ \\
\hline Mean no. of pairwise differences $(\Pi)^{\mathrm{a}}$ & $5.259 \pm 2.557$ & $13.029 \pm 5.949$ & $13.773 \pm 6.216$ \\
\hline Theta $_{\mathrm{s}}\left(\theta_{\mathrm{s}}\right)^{\mathrm{a}}$ & $4.527 \pm 1.350$ & $11.580 \pm 3.413$ & $9.048 \pm 2.354$ \\
\hline Nucleotide diversity $(\pi)$ & $0.014 \pm 0.007$ & $0.034 \pm 0.017$ & $0.036 \pm 0.018$ \\
\hline
\end{tabular}

a Compared for Tajima's D test.

TABLE 4. Neutrality tests and mismatch distribution analyses for $R$. brelichi, $R$. roxellana, and $R$. bieti ${ }^{a}$

\begin{tabular}{lccc}
\hline & $R$. brelichi & $R$. roxellana & $R$. bieti \\
\hline No. of individuals & 141 & 60 & 157 \\
Tajima's D $(P$ value $)$ & $0.464(0.739)$ & $0.424(0.742)$ & $1.589(0.955)$ \\
Fu's $\mathrm{F}_{\mathrm{s}}(P$ value $)$ & $12.597(0.988)$ & $8.452(0.976)$ & $2.095(0.770)$ \\
SSD $(P$ value $)$ & $0.120(0.080)$ & $0.061(0.030)$ & $0.018(0.100)$ \\
$r(P$ value $)$ & $0.347(0.447)$ & $0.074(0.008)$ & $0.012(0.590)$ \\
$\theta_{0}$ & $<0.001$ & $<0.001$ & 2.589 \\
$\theta_{1}$ & 0.554 & 17.783 & 16.826 \\
$\tau$ & 3.250 & 35.113 & 4.207 \\
\hline
\end{tabular}

${ }^{\text {a }} \mathrm{SSD}$, sum of squared deviations; $r$, raggedness index; $\theta_{0}$, population size before expansion; $\theta_{1}$, population size after expansion; $\tau$, age of expansion in units of mutational time.

marized in Table 3. The haplotype diversity for $R$. brelichi $(h=0.457)$ is roughly half of that estimated for $R$. roxellana $(h=0.845)$ and $R$. bieti $(h=0.945)$. For all three species, nucleotide diversities ( $R$. brelichi: $\pi=$ $0.014, R$. roxellana: $\pi=0.034, R$. bieti: $\pi=0.036$ ) are relatively low, especially for $R$. brelichi. The average number of pairwise nucleotide differences ( $\Pi$ ) between individuals amounts to 5.3 in $R$. brelichi, 13.0 in $R$. roxellana and 13.8 in $R$. bieti. The much lower $\Pi$ in $R$. brelichi and the topology of the haplotype network support that there is no genetic population structure in this species.

\section{Demographic history}

The nonsignificant values of the neutrality tests, including both Tajima's $\mathrm{D}$ and Fu's $\mathrm{F}_{\mathrm{S}}$, indicate no departure from the null hypothesis of demographic stability within the $95 \%$ confidence interval (Table 4). This result is further supported by the multimodal patterns of the mismatch distribution of all three species pointing to a stable population size (Fig. 4). The distribution for $R$. brelichi with a very high initial peak (probability of identical haplotypes at around 0.55) may provide weak evidence for a population reduction (Rogers and Harpending, 1992). However, it should be noted that the mismatch distribution is highly influenced by population structure. The multimodal pattern in $R$. roxellana and $R$. bieti, with a prominent second peak for higher numbers of pairwise differences, is most likely caused by their respective population subdivision (discussed in detail in Li et al., 2007; Liu et al., 2007).

The statistical properties of the mismatch distribution under the population expansion model are compared in Table 4. The SSD values of all three species are found to be significant at least at the $90 \%$ confidence level. Only the SSD value for $R$. brelichi may indicate a departure from the estimated demographic model of a sudden pop- ulation expansion, in contrast to the low SSD values for the other two species.

Similarly, the raggedness indices for $R$. roxellana and $R$. bieti are much lower than for $R$. brelichi and thus suggest a relatively good fit of the data to the model of population expansion (Harpending, 1994). However, it has to be noted here that only the raggedness index for $R$. roxellana is significant $(P=0.008)$.

\section{DISCUSSION}

The taxonomic classification and especially the phylogenetic relationships among Rhinopithecus taxa have been debated (Rowe, 1996; Jablonski, 1998a; Pan and Oxnard, 2001). Early studies suggested that the three Chinese species were subspecies of one species ( $R$. roxellana; Ellerman and Morrison-Scott, 1951; Napier and Napier, 1967; Quan and Xie, 1981; Zhang et al., 1992). More recently, however, $R$. bieti and $R$. brelichi were separated from $R$. roxellana on species level (Peng et al., 1988; Jablonski and Peng, 1993; Zhang et al., 1997; Zhang and Ryder, 1998; Groves, 2001; Li et al., 2001; Pan and Oxnard, 2001). The results of our study clearly support the "three Chinese snub-nosed monkey species" hypothesis.

The phylogenetic relationships among $R$. roxellana, $R$. bieti, $R$. brelichi, and the Vietnamese $R$. avunculus are not well defined. Since $R$. strykeri was just recently described, its phylogenetic position within the genus is not explored in detail yet. For the four other species, Jablonski and Peng (1993) suggested a basal position of $R$. avunculus and a sister grouping of $R$. roxellana to a clade consisting of $R$. bieti and $R$. brelichi. Genetic studies based on relatively short mitochondrial DNA fragments allowed no significant resolution among these four species (Zhang and Ryder, 1998; Li et al., 2004; Roos et al., 2007), while recent work using complete mitochondrial genomes revealed a strongly supported branching pattern among them (Yang et al., in press). Accordingly, $R$. avunculus represents indeed the first lineage, but among the Chinese species, $R$. bieti and not $R$. roxellana is basal. Our results support the phylogenetic relationships as suggested by Yang et al. (in press), because the genetic distance between $R$. brelichi and $R$. roxellana is smaller than their distance to $R$. bieti, and also our phylogenetic reconstruction indicates $R$. brelichi and $R$. roxellana as sister taxa. Also in agreement with Yang et al. (in press), divergence age estimates suggest Rhinopithecus differentiation into species in the early Pleistocene. Although our estimates for the MRCAs of $R$. bieti and $R$. roxellana are slightly older, they are in a similar range as those obtained in earlier studies (Li et al., 2007; Liu et al., 2007). According to our estimate, the MRCA of the 

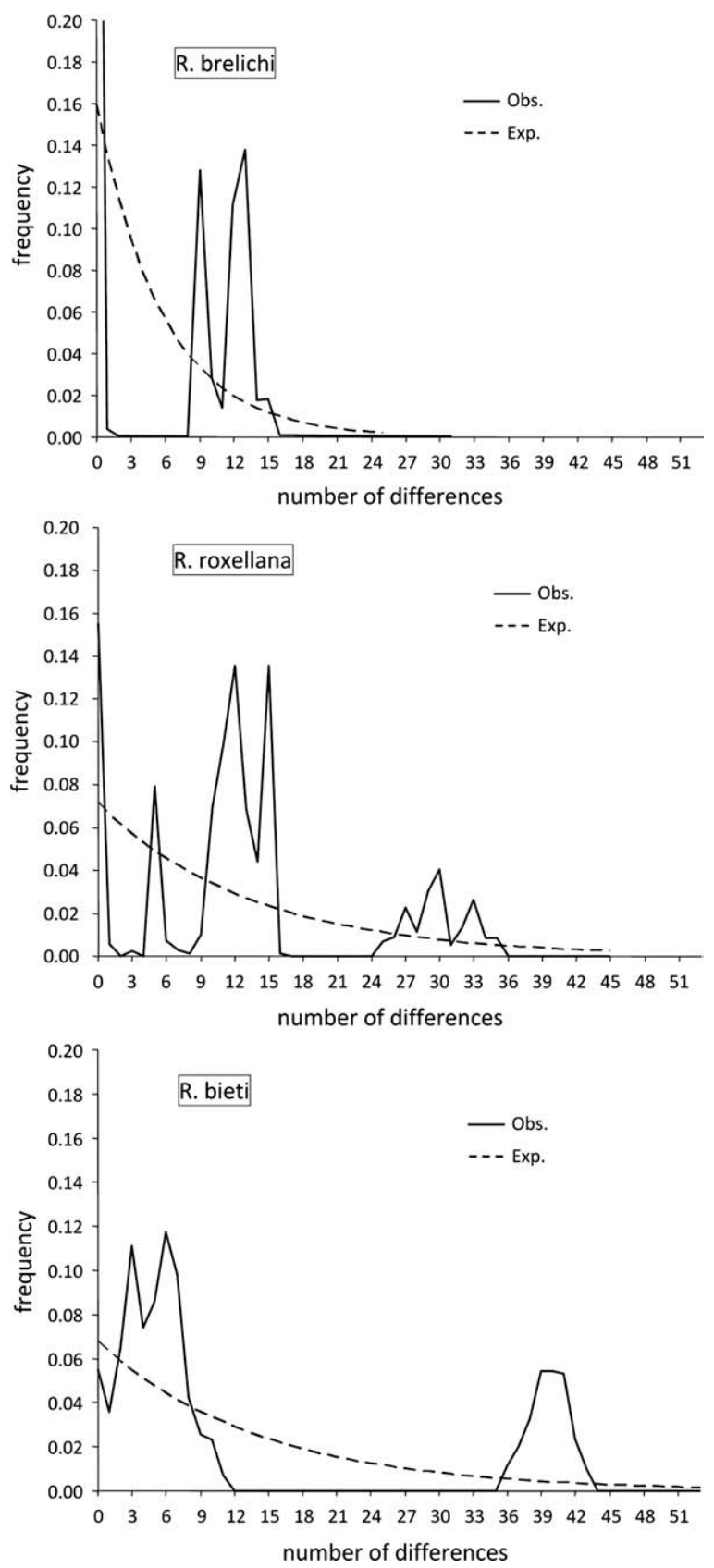

Fig. 4. Mismatch distribution of $R$. brelichi, R. roxellana, and $R$. bieti HVI sequences. Frequency of pairs of individuals (in percent) plotted over the number of nucleotide site differences. ( $R$. roxellana: initial $\theta<0.001$; final $\theta=17.783 ; \tau=35.113$; $R$. bieti: initial $\theta=2.589$; final $\theta=16.826 ; \tau=4.207$; $R$. brelichi: initial $\theta<0.001$; final $\theta=0.554 ; \tau=3.251$ ).

R. brelichi mitochondrial haplotypes occurred more recently than that of the other two species.

As shown in Table 3, the values for genetic diversity (e.g., polymorphic sites, number of haplotypes, haplotype diversity $(h)$, mean number of pairwise differences, nucleotide diversity) for $R$. brelichi are roughly half of that for $R$. roxellana and $R$. bieti. Compared to other primate species, h and $\pi$ for $R$. roxellana and $R$. bieti are similar to, for example, Barbary macaques (Macaca sylvanus, $h$ $=0.872, \pi=0.026$, Modolo et al., 2005) and Arabian hamadryas baboons (Papio hamadryas, $h=0.856$, and $\pi$ $=0.023$, Winney et al., 2004), both with a restricted range and relatively small population. Even more extreme, $h$ and $\pi$ for $R$. brelichi are similar to a species which lives on a small island (Japanese macaque, Macaca fuscata yakui, $h=0.305, \pi=0.015$, Hayaishi and Kawamoto, 2006). Moreover, 102 of the 141 analyzed $R$. brelichi individuals share the same haplotype and the five haplotypes in total differ in 26 polymorphic sites only. All of these suggest that $R$. brelichi today has the lowest genetic variation among the three Chinese snubnosed monkey species.

Both Tajima's D and Fu's $\mathrm{F}_{\mathrm{S}}$ tests do not indicate population expansions or contractions in any of the three Chinese species. Furthermore, mismatch distribution analysis clearly reveals equilibrium in $R$. brelichi. For the other two species, the picture is more complicated, possibly due to their population structure. These results are consistent with earlier studies on the demographic history of $R$. roxellana and $R$. bieti, saying that although different subpopulations probably underwent different demographic changes, the total population remained stable (Li et al., 2007; Liu et al., 2007). In $R$. brelichi, there seems to be neither geographic nor genetic substructuring that could have influenced the analyses of demographic history. Overall, our data suggest population equilibrium also in $R$. brelichi.

Fossils suggest that snub-nosed monkeys were once widespread and experienced range reductions in the late Pleistocene or Holocene (Jablonski, 1998a,b; Quan and Xie, 2002). In the Qing Dynasty (1616-1911), however, snub-nosed monkeys still occurred in 11 provinces of China ( $\mathrm{Li}$ et al., 2002). An additional reduction in population density and distribution occurred, particularly in $R$. brelichi, during the last 400 years (Li et al., 2002). During this period, the human population increased dramatically in southern China (Li et al., 2002) and with it, deforestation and hunting, which further reduced suitable monkey habitat and population sizes (Li et al., 2002). Both, the reduction of habitat and population size, might have led to the observed low genetic diversity of $R$. breli$c h i$. However, the population size reduction is in contrast to our genetic data. One reason for this incongruence might be that the currently surviving population was isolated from others, now extinct $R$. brelichi populations, for a certain time period and might have kept its originally low genetic variability.

Researchers found extensive gene flow between the different subpopulations of $R$. roxellana and $R$. bieti, respectively (Liu et al., 2009; Pan et al., 2009), which might enhance survival under environmental changes or climate oscillations. In contrast, for $R$. brelichi genetic exchange with other subpopulations is impossible. Given the slow reproduction rate, the small total population size, and the low genetic diversity, $R$. brelichi is probably the most vulnerable snub-nosed monkey species in China. Therefore, it is of urgent management interest to collect more data on the genetics, ecology, particularly ranging of the species and about the mating system to assist in the protection of this enigmatic primate species.

\section{ACKNOWLEDGMENTS}

The authors are grateful to the staff of Fanjingshan National Nature Reserve in Guizhou province, China, for helping to collect samples and for supporting this study. The authors also thank Fanjingshan National Na- 
ture Reserve for permits to collect samples and the Forestry Ministry of Guizhou Province, China for research permits. Christiane Schwarz is thanked for her excellent technical assistance in the laboratory, Stephen Nash for the illustrations of the snub-nosed monkeys, and $\mathrm{Mi}$ chael Krützen and Mike Bruford for helpful discussions.

\section{LITERATURE CITED}

Bandelt HJ, Forster P, Röhl A. 1999. Median-joining networks for inferring intraspecific phylogenies. Mol Biol Evol 16:37-48.

Bleisch W, Xie JH. 1998. Ecology and behavior of the Guizhou snub-nosed langur (Rhinopithecus [Rhinopithecus] brelichi), with a discussion of socioecology in the genus. In: Jablonski NG, editor. The natural history of the doucs and snub-nosed monkeys. Singapore: World Scientific Publishing. p 217-239.

Cui LW, Sheng AH, He XC, Xiao W. 2006. Birth seasonality and interbirth interval of captive Rhinopithecus bieti. Am J Primatol 68:457-463.

de Jong G, de Ruiter JR, Haring R. 1994. Genetic structure of a population with social structure and migration. In: Loeschcke V, Tomiuk J, Jain SK, editors. Conservation genetics. Basel: Birkhäuser Verlag. p 147-164.

Dietz JM, Baker AJ, Ballou JD. 2000. Demographic evidence of inbreeding depression in wild golden lion tamarins. In: Yang A, Clark GM, editors. Genetics, demography, and viability of fragmented populations. Cambridge: Cambridge University Press. p 203-211.

Di Fiore A. 2003. Molecular genetic approaches to the study of primate behavior, social organization, and reproduction. Am J Phys Anthropol 122:62-99.

Drummond AJ, Ho SY, Phillips MJ, Rambaut A. 2006. Relaxed phylogenetics and dating with confidence. PLoS Biol 4:e88

Drummond AJ, Rambaut A. 2007. BEAST: Bayesian evolutionary analysis by sampling trees. BMC Evol Biol 7:214.

Ellerman JR, Morrison-Scott TCS. 1951. Checklist of Palaearctic and Indian mammals. London: Trustees of the British Museum (Natural History). p 22-89.

Excoffier L, Lischer HL. 2010. Arlequin suite ver 3.5: a new series of programs to perform population genetics analyses under Linux and Windows. Mol Ecol Res 10:564-567.

Excoffier L, Smouse PE, Quattro JM. 1992. Analysis of molecular variance inferred from metric distances among DNA haplotypes: application to human mitochondrial DNA restriction data. Genetics 131:479-491.

Fu YX. 1997. Statistical tests of neutrality of mutations against population growth, hitchhiking and background selection. Genetics 147:915-925.

Geissmann T, Lwin N, Aung SS, Aung TN, Aung MZ, Hla TH, Grindler M, Momberg F. 2011. A new species of snub-nosed monkey, genus Rhinopithecus Milne-Edwards, 1872 (Primates, Colobinae), from northern Kachin State, northeastern Myanmar. Am J Primatol 73:96-107.

Groves CP. 2001. Primate taxonomy. Washington, DC: Smithsonian Institution Press.

Hall TA. 1999. BioEdit: a user-friendly biological sequence alignment editor and analysis program for Windows 95/98/NT. Nucleic Acids Symp 41:95-98.

Harpending HC. 1994. Signature of ancient population growth in a low-resolution mitochondrial DNA mismatch distribution. Hum Biol 66:591-600.

Hartl DL, Clark AG. 2007. Principles of population genetics. Sunderland, MA: Sinauer.

Hayaishi S, Kawamoto Y. 2006. Low genetic diversity and biased distribution of mitochondrial DNA haplotypes in the Japanese macaque (Macaca fuscata yakui) on Yakushima Island. Primates 47:158-164.

Hoeglund J. 2009. Evolutionary conservation genetics. Oxford: Oxford University Press.

Huelsenbeck JP, Ronquist F, Nielsen R, Bollback JP. 2001. Bayesian inference of phylogeny and its impact on evolutionary biology. Science 294:2310-2314.
IUCN. 2010. The IUCN red list of threatened species. Available at: http://www.iucnredlist.org.

Jablonski NG. 1998a. The response of catarrhine primates to Pleistocene environmental fluctuations in East Asia. Primates 39:29-37.

Jablonski NG. 1998b. The evolution of the doucs and snubnosed monkeys and the question of the phyletic unity of the odd-nosed colobines. In: Jablonski NG, editor. The natural history of the doucs and snub-nosed monkeys. Singapore: World Scientific Publishing. p 13-52.

Jablonski NG, Peng YZ. 1993. The phylogenetic relationships and classification of the doucs and snub-nosed langurs of China and Vietnam. Folia Primatol 60:36-55.

Ji WZ, Zou RJ, Shang EY, Zhou HW, Yang SC, Tian BP. 1998. Maintenance and breeding of Yunnan snub-nosed monkeys (Rhinopithecus [Rhinopithecus] bieti) in captivity. In: Jablonski NG, editor. The natural history of the doucs and snubnosed monkeys. Singapore: World Scientific Publishing. p 323-335.

Li BG, Pan RL, Oxnard CE. 2002. Extinction of snub-nosed monkeys in China during the past 400 years. Int J Primatol 23:1227-1244.

Li HP, Meng SJ, Men ZM, Fu YX, Zhang YP. 2003. Genetic diversity and population history of golden monkeys (Rhinopithecus roxellana). Genetics 164:269-275.

Li M, Liang B, Feng ZJ, Tamate HB. 2001. Molecular phylogenetic relationships among Sichuan snub-nosed monkeys (Rhinopithecus roxellana) inferred from mitochondrial cytochrome b gene sequences. Primates 42:153-160.

Li M, Liu ZJ, Gou JX, Gou JX, Ren BP, Pan RL, Su YJ, Funk SM, Wei FW. 2007. Phylogeography and population structure of the golden monkeys (Rhinopithecus roxellana): inferred from mitochondrial DNA sequences. Am J Primatol 69:11951209.

Li M, Wei FW, Huang CM, Pan RL, de Ruiter J. 2004. Phylogeny of snub-nosed monkeys inferred from mitochondrial DNA. cytochrome $\mathrm{b}$, and $12 \mathrm{~S}$ rRNA sequences. Int $\mathrm{J}$ Primatol 25:861-873.

Liang B, Qi HJ, Zhang SY, Ren BP. 2001. Developmental traits of captive Sichuan snub-nosed monkeys (Rhinopithecus roxellana) at different age stages. Acta Zool Sinica 47:381-387.

Librado P, Rozas J. 2009. DnaSP version 5: a software for comprehensive analysis of DNA polymorphism data. Bioinformatics 25:1451-1452.

Liu ZJ, Ren BP, Wei FW, Long YC, Hao YL, Li M. 2007. Phylogeography and population structure of the Yunnan snubnosed monkey (Rhinopithecus bieti) inferred from mitochondrial control region DNA sequence analysis. Mol Ecol 16:3334-3349.

Liu ZJ, Ren BP, Wu RD, Zhao L, Hao YL, Wang BS, Wei FW, Long YC, Li M. 2009. The effect of landscape features on population genetic structure in Yunnan snub-nosed monkeys (Rhinopithecus bieti) implies an anthropogenic genetic discontinuity. Mol Ecol 18:3831-3846.

MacKinnon J. 2008. Order primates. In: Smith AT, Xie Y, editors. A guide to the mammals of China. Princeton: Princeton University Press. p 158-172.

Mittermeier RA, Wallis J, Rylands AB, Ganzhorn JU, Oates JF, Williamson EA, Palacios E, Heymann EW, Kierulff MCM, Long Y, Supriatna J, Roos C, Walker S, Cortés-Ortiz L, Schwitzer C. 2009. Primates in peril: the world's 25 most endangered primates 2008-2010. Primate Conserv 24:1-57.

Modolo L, Salzburger W, Martin RD. 2005. Phylogeography of Barbary macaques (Macaca sylvanus) and the origin of the Gibraltar colony. Proc Natl Acad Sci USA 102:7392-7397.

Morin PA, Chambers KE, Boesch C, Vigilant L. 2001. Quantitative polymerase chain reaction analysis of DNA from noninvasive samples for accurate microsatellite genotyping of wild chimpanzees (Pan troglodytes verus). Mol Ecol 10:18351844.

Napier JR, Napier PH. 1967. A handbook of living primates. London: Academic Press.

Nei M. 1977. F-statistics and analysis of gene diversity in subdivided populations. Ann Hum Genet 41:225-233. 
Nei M. 1987. Molecular evolutionary genetics. New York: Columbia University Press.

Nei M, Li WH. 1979. Mathematical model for studying genetic variation in terms of restriction endonucleases. Proc Natl Acad Sci USA 76:5269-5273.

Osterholz M, Walter L, Roos C. 2008. Phylogenetic position of the langur genera Semnopithecus and Trachypithecus among Asian colobines, and genus affiliations of their species groups. BMC Evol Biol 8:58.

Pan D, Hu HX, Meng SJ, Men ZM, Fu YX, Zhang YP. 2009. A high polymorphism level in Rhinopithecus roxellana. Int $\mathrm{J}$ Primatol 30:337-351.

Pan RL, Oxnard CE. 2001. Cranial morphology of the golden monkey (Rhinopithecus) and douc langur (Pygathrix nemaeus). Hum Evol 16:199-223.

Peakall R, Smouse PE. 2006. GENALEX 6: genetic analysis in Excel: population genetic software for teaching and research. Mol Ecol Notes 6:1288-1295.

Peng YZ, Ye ZZ, Zhang YP, Pan RL. 1988. The classification and phylogeny of snub-nosed monkey Rhinopithecus spp. based on gross morphological characters. Zool Res 9:239-248.

Posada D. 2008. jModelTest: phylogenetic model averaging. Mol Biol Evol 25:1253-1256.

Qi XG, Li BG, Ji WH. 2008. Reproductive parameters of wild female Rhinopithecus roxellana. Am J Primatol 70:311-319.

Quan GQ, Xie JH. 1981. Notes on Rhinopithecus roxellanae brelichi (Thomas). Acta Theriol Sinica 1:113-116.

Quan GQ, Xie JY. 2002. Research on the golden monkey. Shanghai: Science and Education Publishing House.

Rambaut A. 2008. FigTree: Tree figure drawing tool, version 1.2.2. Available at: http://tree.bio.ed.ac.uk/software/figtree/.

Rambaut A, Drummond AJ. 2007. Tracer: MCMC trace analysis tool, version 1.4.1. Available at: http://tree.bio.ed.ac.uk/software/tracer/.

Rogers AR, Harpending H. 1992. Population growth makes waves in the distribution of pairwise genetic differences. Mol Biol Evol 9:552-569.

Ronquist F, Huelsenbeck JP. 2003. MrBayes 3: Bayesian phylogenetic inference under mixed models. Bioinformatics 19: $1572-1574$

Roos C, Thanh VN, Walter L, Nadler T. 2007. Molecular systematics of Indochinese primates. Vietn J Primatol 1:41-53.

Rowe N. 1996. The pictorial guide to the living primates. East Hampton: Pogonias Press.

Santos C, Montiel R, Sierra B, Bettencourt C, Fernandez E, Alvarez L, Lima M, Abade A, Aluja MP. 2005. Understanding differences between phylogenetic and pedigree-derived mtDNA mutation rate: a model using families from the Azores islands (Portugal). Mol Biol Evol 22:1490-1505.

Schmidt D, Pool J. 2002. The effect of population history on the distribution of the Tajima's D statistic. New York: Cornell University Press.

Schneider S, Excoffier L. 1999. Estimation of demographic parameters from the distribution of pairwise differences when the mutation rates vary among sites: application to human mitochondrial DNA. Genetics 152:1079-1089.

Soares P, Ermini L, Thomson N, Mormina M, Rito T, Röhl A, Salas A, Oppenheimer S, Macaulay V, Richards MB. 2009.
Correcting for purifying selection: an improved human mitochondrial molecular clock. Am J Hum Genet 84:740-759.

Spielman D, Brook BW, Frankham R. 2004. Most species are not driven to extinction before genetic factors impact them. Proc Natl Acad Sci USA 101:15261-15264.

Swofford, DL. 2003. PAUP*: phylogenetic analysis using parsimony (* and other methods), version 4.0b10. Sunderland, MA: Sinauer.

Tajima F. 1989. Statistical method for testing the neutral mutation hypothesis by DNA polymorphism. Genetics 123:585-595. van Oosterhout C, Hutchinson WF, Wills DPM, Shipley P. 2004. Micro-Checker: software for identifying and correcting genotyping errors in microsatellite data. Mol Ecol Notes 4:535-538.

Wakeley J. 1997. Using the variance of pairwise differences to estimate the recombination rate. Genet Res 69:45-48.

Waits LP, Luikart G, Taberlet P. 2001. Estimating the probability of identity among genotypes in natural populations: cautions and guidelines. Mol Ecol 10:249-256.

Winney BJ, Hammond RL, Macasero W, Flores B, Boug A, Biquand V, Biquand S, Bruford MW. 2004. Crossing the Red Sea: phylogeography of the hamadryas baboon, Papio hamadryas hamadryas. Mol Ecol 13:2819-2827.

Wright S. 1969. Evolution and the genetics of populations. II: The theory of gene frequencies. Chicago: University of Chicago Press.

Wright S. 1978. Evolution and the genetics of populations. IV: the variability within and among natural populations. Chicago: University of Chicago Press.

Wu G, Wang HC, Fu HW, Zhao JZ, Yang YQ. 2004. Habitat selection of Guizhou golden monkey (Rhinopithecus roxellanae brelichi) in Fanjing Mountain Biosphere Reserve, China. J Forest Res 15:197-202.

Yang M, Sun D, Zinner D, Roos C. 2009. Reproductive parameters in Guizhou snub-nosed monkeys (Rhinopithecus brelichi). Am J Primatol 71:266-270.

Yang M, Nadler T, Zinner D, Roos C. The evolutionary history of odd-nosed monkeys. In: Tan C, Grüter C, Wright B, editors. Odd-nosed monkeys: recent advances in the study of the forgotten colobines (in press).

Yang YQ, Lei XP, Sun DY, Yang CD, He RT, Zhang WY. 2002. Ecology of the wild Guizhou snub-nosed monkey. Guiyang: Guizhou Scientific Institute Press.

Zhang SY, Liang B, Wang LX. 2000. Seasonality of mating and births in captive Sichuan golden monkeys (Rhinopithecus roxellanae). Am J Primatol 51:265-269.

Zhang YP, Ryder OA, Fan Z, Zhang H. 1997. Sequence variation and genetic diversity in giant panda. Sci China C Life Sci 27:139-144.

Zhang YP, Ryder OA. 1998. Mitochondrial cytochrome b gene sequences of Old World monkeys: with a special reference on evolution of Asian colobines. Primates 39:39-49.

Zhang YZ, Quan GQ, Zhao TG, Southwick CH. 1992. Distribution of primates (except Macaca) in China. Acta Theriol Sinica 12:81-95.

Zwickl DJ. 2006. Genetic algorithm approaches for the phylogenetic analysis of large biological sequence datasets under the maximum-likelihood criterion. $\mathrm{PhD}$ thesis. The University of Texas at Austin. 


\section{General Discussion}

My thesis is divided in three major parts which constitute a top-down approach of analyses of phylogenetic relationships and population genetic parameters in leaf monkeys (Colobinae). The first part of my thesis provides a comprehensive phylogeny of the colobines genera by combining different genetic markers. In the second part, my thesis focuses on the evolutionary history of one major radiation of the Colobinae, the odd-nosed monkeys. Based on complete mitochondrial genome sequence data from nine odd-nosed monkey species I reconstructed the phylogenetic relationships among genera and species and estimated respective divergence ages. Finally, a population genetic analysis was performed for one of the Chinese snub-nosed monkey species, the endangered Guizhou snub-nosed monkeys (Rhinopithecus brelichi). I assessed the population genetic variation and demographic history of this species in comparison with already existing data of its Chinese congenerics.

\section{Summary of the main findings}

In the first part of my thesis (Chapter 1 ) a combination of mitochondrial genome data (ca. 16,600 bp), sequence information from five autosomal loci (ca. 5,300 bp), one $X$ chromosomal locus (4,000 bp) and six $Y$ chromosomal loci (ca. 4,000 bp), and the presence/absence pattern of the mobile element of the ten colobines genera were used to reconstruct the phylogenetic relationships among all Colobinae genera. Phylogenies based on different genetic markers show a similar topology, but several incongruences were also observed. First, the African colobines constitute a monophyletic clade based on the nuclear and mitochondrial sequence data, while according to the mobile elements data African colobines are paraphyletic with the Piliocolobus/Procolobus clade closer related to the Asian colobines than to Colobus. Second, nuclear sequence data put Presbytis as basal to all Asian colobines, whereas the mobile elements indicate a sister clade relationship between Presbytis and the odd-nosed monkeys. Third, the mitochondrial data reveal Semnopithecus, Presbytis + Trachypithecus and the odd-nosed monkeys as three-way lineages, while the nuclear data suggest a distinct Presbytis lineage (see above) and a Semnopithecus + Trachypithecus clade. Although various explanations are possible for the observed incongruences, ancient 
hybridization events for at least two cases are most likely. For the odd-nosed monkey genera, identical branching patterns with Rhinopithecus as most basal lineage, followed by Pygathrix and a sister grouping of Nasalis and Simias was obtained from all data sets.

The second part of my thesis (Chapter 2) was a mitogenomic analysis of the phylogenetic relationships and divergence times within the odd-nosed monkey clade and included nine of the ten recognized species. $R$. strykeri was not included here because this species was only recently discovered in Myanmar and material for a genetic analysis was not available. My study strongly supports as in Chapter 1 a monophyly of the odd-nosed monkeys and indicates that Rhinopithecus occurs as basal, whereas Pygathrix forms a sister lineage to the Nasalis + Simias clade. The diversification of odd-nosed monkeys into genera started ca. 6.85 mya and ended 1.98 mya with the split between Nasalis and Simias. Subsequent radiations in Rhinopithecus and Pygathrix occurred during the Pleistocene. Within Rhinopithecus, my analysis reveals that $R$. roxellana $+R$. brelichi form a sister clade to $R$. bieti, while $R$. avunculus occurs as the most basal taxon. In Pygathrix, $P$. nigripes is basal to the $P$. nemaeus $+P$. cinerea clade.

In part 3 of my thesis (Chapter 3 ) I performed a population genetic study on $R$. brelichi for which I used the hypervariable region I of the mitochondrial control region. The data were compared with respective published information from the two other Chinese snub-nosed monkey species ( $R$. roxellana, $R$. bieti). My results indicate significant genetic differences among the three species. Rather low nucleotide diversity was detected in all three Chinese snub-nosed species, with the lowest genetic diversity in $R$. brelichi. Among $141 R$. brelichi individuals only five haplotypes were found and one occurred in 102 individuals. Tajima's D, Fu's $F_{s}$ and mismatch distribution tests all showed an equilibrium population in the demographic history of the wild $R$. brelichi population. The MRCAs of $R$. bieti, $R$. roxellana and $R$. brelichi lived ca. 1.33, 0.91 and 0.36 mya, respectively.

\section{The evolutionary history of colobines genera}

In a comprehensive approach we investigated the evolutionary history of colobines by combining maternal-, paternal- and biparental-inherited molecular 
markers from all ten colobines genera. Hence, this study represents the most detailed insight into the evolutionary history of colobines.

The phylogenetic trees derived from the analysis of different molecular markers are often incongruent (Grechko, 2002; Tosi et al., 2003). A few discordances have been found in the phylogenetic topologies in this study as well. Our mitochondrial and nuclear data both support a reciprocal monophyletic origin of the African and the Asian colobines, which is in agreement with morphological studies (Groves, 2001; Napier and Napier, 1967; Szalay and Delson, 1979) and earlier molecular studies (Collura et al., 1996; Messier and Stewart, 1997; Zhang and Ryder, 1998; Page et al., 1999; Bigoni et al., 2003, 2004; Xing et al., 2005). In contrast, the mobile elements propose a paraphyly of African colobines (Groves, 1989; Peng et al., 1993; Jablonski, 1998c; Osterholz et al., 2008).

Discordant phylogenetic relationships among genera based on different markers have been explained in past studies by using insufficient data, homoplasy, incomplete lineage sorting (ILS), or hybridization (Philippe and Laurent, 1998; Barton, 2001; Nichols, 2001; Funk and Omland, 2003; Avise, 2004; Seehausen, 2004; McCracken and Sorenson, 2005; Xing et al., 2005; Pollard et al., 2006; Koblmüller et al., 2007; Petit and Excoffier, 2009). For the mobile elements, we did not found any inconsistent elements; therefore, ILS is unlikely to be an explanation for our findings. Homoplasy is usually regarded as not relevant for the analyses of mobile elements (Okada, 1991; Schmitz et al., 2005; Ray et al., 2006). Overall, inadequate data, homoplasy, ILS could not provide sufficient explanations for the observed incongruence in this study. Moreover, mobile elements as molecular-cladistic marker are more reliable than pure sequence data (Kazazian, 2004; Osterholz et al., 2008), thus, the discordant pattern most likely is caused by ancestral hybridization between the ancestor of Colobus and the Piliocolobus/Procolobus lineage. This view is also supported by biological data (Davies and Oates, 1994; Groves, 2001). In contrast to Colobus, the females of Piliocolobus/Procolobus tend to leave their natal groups (Newton and Dunbar, 1994). Colobus males are normally bigger than Piliocolobus/Procolobus males, thus, Colobus males increase their chance to hybridize with Piliocolobus/Procolobus females. Moreover, the two ancestral genera occur over wide ranges of their distribution in sympatry (Davies and Oates, 1994; Newton and Dunbar, 1994; Groves, 2001); hence, it can be assumed that ancestral hybridization events were basically possible. 
For the Asian colobines, the phylogenetic relationships among the odd-nosed monkeys were the same from all markers. Five mobile elements adduced sufficient evidence for the monophyly of the odd-nosed monkeys. The view on their monophyletic origin was already earlier supported by mitochondrial sequence data (Sterner et al., 2006). Among odd-nosed monkeys, mobile elements also support the basal position of Rhinopithecus and the grouping of Nasalis with Simias. However, the phylogenetic affiliations within the leaf monkeys and langurs are major parts of a debate. In our study, nuclear sequence data and mobile elements support a paraphyly of langurs and a close relationship of Semnopithecus and Trachypithecus. The morphological characteristics support the idea of a sister clade relationship between Semnopithecus and Trachypithecus (Brandon-Jones, 1984; Strasser and Delson, 1987; Groves, 2001). On the contrary, the mitochondrial sequence data provide a connection between Presbytis and Trachypithecus. Avise (2004) demonstrated that mitochondrial DNA was likely to sort faster than nuclear DNA because the effective population size of mitochondrial DNA was only a quarter of autosomal genes. If ILS was the reason for the observed incongruent gene trees, the mitochondrial divergence between respective genera should be earlier than the nuclear splitting times. However, the divergence time calculated in this study denied this case (Presbytis - Trachypithecus: 8.12 mya nuclear vs. 7.45 mya mitochondrial). Thus, ancestral hybridization is the most likely plausible explanation for the incongruent pattern. In general, hybridization is possible due to partial sympatry (Davies and Oates, 1994; Newton and Dunbar, 1994; Groves, 2001). Semnopithecus males are larger than Trachypithecus (Davies and Oates, 1994). Semnopithecus males leave their natal group and might hybridize with Trachypithecus females. By backcrossing with Semnopithecus males over a rather long time period, the Trachypithecus accumulated nuclear material of Semnopithecus, although the mitochondrial genome remained Trachypithecus-like.

\section{The evolutionary history of odd-nosed monkey species}

The second part of my thesis presents a detailed and updated view into the evolutionary history of odd-nosed monkeys on species level. Since previous studies on the evolutionary history of odd-nosed monkeys only used short mitochondrial fragments and/or did not include all species (Wang et al., 1997; Zhang and Ryder, 1998; Li et al., 2001; Li et al., 2004; Sterner et al., 2006; Whittaker et al., 2006; Li et al., 
2007; Liu et al., 2007; Osterholz et al., 2008; Ting et al., 2008; Li et al., 2011; Perelman et al., 2011), my study provides a phylogeny and respective divergence time estimates based on the complete mitochondrial genome from nine of ten odd-nosed monkey species. $R$. strykeri was not included in this study because this species was only recently described (Geissmann et al., 2011) and genetic material was not yet available.

The divergence time of Simias and Nasalis in the early Pleistocene occurred as most speciation events within Rhinopithecus and Pygathrix. My data points in the direction that Simias should not be recognized as distinct genus and respectively included in Nasalis. These results are in agreement with findings from several previous studies (Delson et al., 2000; Whittaker et al., 2006). Furthermore, my data suggest that the genus Rhinopithecus contains at least four species $(R$. avunculus, $R$. roxellana, $R$. bieti, $R$. brelichi) and that the genus Pygathrix comprises three species ( $P$. nigripes, $P$. cinerea, $P$. nemaeus), because the estimated divergence times among them were similar. The hypothesis that $R$. avunculus represents a separate subgenus Presbyticus (Boonratana and Canh, 1998; Chaplin and Jablonski, 1998; Zhang and Ryder, 1998; Jablonski, 1998) is not supported by my analysis. Genetic data for the newly described $R$. strykeri is not available yet, but it clearly differs from its congenerics by various morphological characters (Geissmann et al., 2011).

By combining our knowledge of phylogeny, divergence time and palaeoenvironmental data, the following phylogeographic scenario for odd-nosed monkeys can be envisioned. Since fossil colobines were found in Eurasia at the end of the Miocene, the ancestor(s) of the Asian colobines probably invaded Eurasia via an emerging land bridge connecting Africa and the Arabian Peninsula and dispersed into Asia (Whybrow, 1992; Stewart and Disotell, 1998; Delson, 2000). The Hengduan Mountains in the border region of today's Myanmar, India and China have been identified as a possible diversification hotspot. Thus, the origin of the odd-nosed monkeys might have been here (Peng et al., 1993; Jablonski, 1998b). In this area, all larger south-eastern rivers (Mekong, Salween, and Yangtze) rise and exist here at least since the early Miocene (Hallet and Molnar, 2001). These rivers have been well known as barriers for arboreal primates (Meijaard and Groves, 2006). After the langur progenitor separated from the odd-nosed monkeys' ancestor in the latest Miocene, the odd-nosed monkeys successively separated and migrated from China to the south and expanded their range into Indochina and Sundaland via a land bridge during periods of 
lower sea levels. Due to selective pressures in various environments, the odd-nosed monkeys chose a wide range of altitudinal and climatic zones and different types of vegetation (Peng et al., 1993). The radiation of Rhinopithecus started in the early Pleistocene probably caused by the reduction and the fragmentation of suitable habitat occurring during that time (Pan and Jablonski, 1987; Jablonski, 1992; Pan, 1995; Jablonski, 1998c; Hartwig, 2002). The divergence time between $P$. nemaeus $+P$. cinerea that I estimated is similar to the divergence time of the species splits among crested gibbons (Thinh et al. 2010) and among limestone langurs (Perelman et al., 2011), thus, the split of Pygathrix most likely was trigged by similar changes in habitat as shrinking forests due to climate changes. Finally, the ancestors of the Nasalis + Simias lineage immigrated into Sundaland most likely during periods of low sea levels (Miller et al., 2005). Although currently Nasalis lives on Borneo and Simias is distributed on the Mentawai Islands, Simias or at least an extinct progenitor of Simias and Nasalis might have existed also on Sumatra. This can be assumed because according to my analysis, gene flow between the two lineages was possible until the end of the early Pleistocene.

\section{Limited genetic variation of Guizhou snub-nosed monkeys ( $R$. brelichi)}

Population genetic data were previously only available for $R$. roxellana and $R$. bieti (Li et al. 2007; Liu et al. 2007). My study presents the first comparative population genetic study on all three Chinese snub-nosed monkey species.

Theoretical considerations suggest that genetic diversity is related to population size because a small population harbours less genetic variation and loses genetic variation faster than larger ones (Wright, 1969, 1978; Frankham et al., 1995; Höglund, 2009). The fossil records indicated that the Chinese snub-nosed monkeys were once widespread in Asia (Li et al., 2002). They experienced a significant range reduction in the late Pleistocene or Holocene according to fossil records (Jablonski 1998c; Quan and Xie 2002). During the last 400 years, their ranges became highly fragmented due to a dramatic human population increase in southern China, which led to accelerating deforestation and hunting activities. The current populations of snub-nosed monkeys survived only in restricted areas (Li et al., 2002). Yang et al. (2002) reported that $R$. brelichi consists of only one population with roughly 800 individuals living in the core zone of Fanjingshan National Nature Reserve. In my study, the analysis of 
mitochondrial DNA sequence data revealed rather low nucleotide diversities ( $\pi)$ for all three Chinese snub-nosed monkey species and the lowest genetic diversity for $R$. brelichi. A low $\pi$ can also be found in other primates with a restricted range and a relatively small population size such as Papio hamadryas in Saudi Arabia $(\pi=0.023$ : Winney et al., 2004) and Macaca sylvanus in Morocco ( $\pi=0.026$ : Modolo et al., 2005). Among the three Chinese snub-nosed monkey species, $R$. brelichi has the smallest population and the most restricted distribution (Bleisch and Xie, 1998; Groves, 2001; Yang et al., 2002; Wu et al., 2004; MacKinnon, 2008; IUCN, 2011). Therefore, the expected low genetic diversity in $R$. brelichi is not surprising. The genetic diversity indices of $R$. brelichi ( $\mathrm{h}=0.457 ; \pi=0.014$ ) were similar to those of Japanese macaques (Macaca fuscata yakui, $h=0.305 ; \pi=0.015$ ), which only lives on a small island (Hayaishi and Kawamoto, 2006). The isolation of the habitat due to historical environmental alterations and/or the recent human activities in the last centuries might provide an explanation for the low genetic diversity in all Chinese snub-nosed monkeys, in particular for $R$. brelichi.

However, analysis of mitochondrial data did not show that the population of $R$. brelichi experienced a recent bottleneck or contraction. The genetic impoverishment most likely was trigged by a population fragmentation and partial extinctions during the last centuries. The only existing $R$. brelichi population might have preserved its original low genetic variability for a certain time period. Additionally, a small population looses genetic variation not only because of a recent bottleneck but also due to genetic drift (Hartl and Clark, 2007; Hamilton, 2009). Genetic drift increases as the population size decreases (Hamilton, 2009). Therefore, genetic drift might cause the low genetic diversity in $R$. brelichi population as well.

\section{Implications for conservation}

All three Chinese snub-nosed monkeys ( $R$. roxellana, $R$. bieti and R. brelichi) are classified as "Endangered" by the IUCN Red List (IUCN, 2011). R. brelichi has the lowest genetic diversity hitherto reported for Chinese snub-nosed monkeys. Gene flow among subpopulations via migration of individuals is one important way to preserve and support the recovery of the genetic diversity within a population (Usher, 1997; Fox and Wolf, 2006) and extensive gene flow was found among subpopulations of $R$. roxellana and $R$. bieti, respectively (Liu et al., 2009; Pan et al., 2009). However, current 
knowledge indicates that for $R$. brelichi only one population exists (Yang et al., 2002). Therefore, an increase of genetic diversity through immigration of individuals from other populations in this species is not possible. The collected samples represented roughly $20 \%$ of the entire known population of $R$. brelichi and they revealed only five haplotypes. A population with low genetic diversity in general has lower adaptive potentials to follow possible environmental changes (Frankel and Soule, 1981; Simberloff, 1988; Barrett and Kohn, 1991; Ellstrand and Elam, 1993; Lande and Shannon, 1996). Furthermore, a study on reproductive pattern of $R$. brelichi disclosed that this species has the slowest reproduction (longest inter-birth interval and the latest age at the first birth) compared with the other two Chinese species (Ji et al., 1998; Zhang et al., 2000; Liang et al., 2001; Cui et al., 2006; Qi et al., 2008; Yang et a., 2009). Summarizing all these results, $R$. brelichi is probably the most vulnerable Rhinopithecus species of China. Accordingly, recovery of the population after suffering natural disasters, environmental changes or a disease can be expected to be rather slow. Any further loss of individuals due to hunting or habitat conversion would have even more dramatic effects than in the other two species. It most likely may not reach the necessary threshold for a sustainable population in time.

The current threats for $R$. brelichi are mainly caused by the habitat conversion induced by human economic activities. Accordingly, habitat protection and the elimination or the balancing of any further human economic activities is a major premise in protecting this species. Nonetheless, further research should collect more samples covering the whole distribution of $R$. brelichi to design effective conservation guidelines.

\section{Outlook}

The evolutionary relationships of the odd-nosed monkeys have been complemented and updated in my study. Future analyses should include $R$. strykeri to complete the phylogenetic relationships within the odd-nosed monkeys. Nuclear data should be applied as well to fully elucidate the phylogenetic relationships among oddnosed monkey species, and probably to uncover possible ancient hybridization events among them. 
In my study I was able to demonstrate a low level of genetic diversity in the wild population of $R$. brelichi, and a population equilibrium in this species based on mitochondrial data. Mitochondria are maternally inherited and therefore represent only one of many molecular tracings in the evolutionary histories of organisms. Therefore, nuclear DNA analyses (microsatellites, SNPs) are required to complement the analysis of genetic diversity and demographic history of $R$. brelichi. Furthermore, population genetic data are also required for $R$. avunculus and $R$. strykeri, and other members of the odd-nosed monkeys.

In the near future more samples from the southern part of the range of $R$. brelichi should be collected. These samples could be utilized to corroborate the results of my population genetic study and to test whether the remaining population of $R$. brelichi consists of more than one subpopulation.

\section{Conclusion}

My thesis shows that extended sequence data provide much better resolution of phylogenetic relationships than only short fragments. This is in particularly true for the herein analysed mitochondrial genome data. Early studies using only short fragments of the mitochondrial genome (Zhang and Ryder, 1998; Li et al., 2004; Roos, 2004; Whittaker et al., 2006; Roos et al., 2007) revealed no or only low resolution for various relationships among odd-nosed monkeys and species of the genus Rhinopithecus. Another good example for the increased resolution for phylogenetic relationships among taxa are the gibbons. While Thinh et al. (2010) with analysing only one mitochondrial gene revealed only low support for relationships among genera and Hylobates species, Chan et al. (2010) by using complete mitochondrial genome data resolved respective relationships with significance. Moreover, the analysis of differently inherited marker systems has proven to be useful to uncover hybridization events, although the therefore required incongruent tree topologies might have been caused by alternatives. Accordingly, testing alternatives is necessary.

Also for population genetic analysis, mitochondrial DNA in particular variable regions as the hypervariable region I of the control region can provide detailed insights into the evolutionary history of a species. However, mitochondrial DNA is only 
maternally inherited. Hence, to obtain a complete picture of the evolutionary history of a species, also paternally and biparentally inherited markers should be analyzed. These could shed light on male-mediated gene flow and socio-biological events in a species, which are otherwise not traceable by field observations. Finally, information about the evolutionary history and in particular the population genetic structure of a species or population provides helpful information for the conservation management of a species, which is of great importance of taxa with small habitat and small populations as in the case of the Guizhou snub-nosed monkey. 


\section{Acknowledgements}

I am deeply indebted to my supervisors Prof. Lutz Walter, Prof. Eckhard W. Heymann and Prof. Peter M. Kappeler, for giving me the opportunity to embark on this study, for their continuous advice, their support during this study and for their efforts as referees for this dissertation.

I extend my thanks to the Fanjingshan National Nature Reserve for the excellent logistical support in the field, in particular to Yeqin Yang and Xiaoping Lei. Thanks also to the Beijing Zoo for providing samples and granting a generous allowance to use them for this study.

At the DPZ I would like to thank everyone for making my time there so pleasant, especially Christiane Schwartz for all her invaluable help in doing laboratory work and her endless patience. Thanks to Nicole Otto, Nico Westphal, Christina Albrecht and Meike Hermes for their cooperative support as well.

I deeply thank Dr. Dietmar Zinner for his endless advice and support in writing papers, for applying for the camera-trapping project, for doing fieldwork and fruitful discussions. Furthermore I am thankful to Dr. Markus Brameier for his assistance in statistical matters and to Gisela Fickenscher for providing substantial input to my publications.

My special thanks goes to Dr. Christian Roos for spending a lot of his time with me and for sharing his experience in invaluable discussions at various stages of my study, for which I am extremely grateful. I will never forget him drafting the proposal for this project and offering me the opportunity to work at the DPZ as a guest in 2007. I sincerely thank him for his guidance, for his infinite patience, and for always believing in me.

Genuine thanks to my parents for always backing and understanding my decisions. Finally many thanks to my husband Alois who always encouraged and supported me in fulfilling my dream. 


\section{References}

Arnold ML, Meyer A. 2006. Natural hybridization in primates: one evolutionary mechanism. Zoology 109:261-276.

Avise JC. 2004. Molecular Markers, Natural History, and Evolution. Sunderland, MA: Sinauer Associates.

Avise JC, Hamrick JL. 1995. Conservation genetics, case histories from nature. New York: Chapman and Hall.

Bandelt HJ, Forster P, Röhl A. 1999. Median-joining networks for inferring intraspecific phylogenies. Mol Biol Evol 16:37-48.

Barrett SCH, Kohn JR. 1991. Genetic and evolutionary consequences of small population size in plants: implications for conservation. In: Falk DA, Holsinger KE, editors. Genetics and Conservation of Rare Plants. New York: Oxford University Press. p 3-30.

Barton NH. 2001. The role of hybridization on evolution. Mol Ecol 10:551-568.

Batzer MA, Deininger PL. 1991. A human-specific subfamily of Alu sequences. Genomics 9:481-487.

Benefit BR, McCrossin ML. 2002. The Victoriapithecidae, Cercopithecoidea. In: Hartwig WC, editor. The Primate Fossil Record. Cambridge: Cambridge University Press. p 241-253.

Bennett EL, Davies AG. 1994. The ecology of Asian colobines. In: Davies AG, Oates JF, editors. Colobine monkeys: their ecology, behavior, and evolution. Cambridge: Cambridge University Press. p 129-171.

Benson DA, Boguski MS, Lipman DJ, Ostell J, Ouellette BF. 1998. Genbank. Nucleic Acids Res 26:1-7.

Bigoni F, Stanyon R, Wimmer R, Schempp W. 2003. Chromosome painting shows that the proboscis monkey (Nasalis larvatus) has a derived karyotype and is phylogenetically nested within Asian colobines. Am J Primatol 60 (3):85-93.

Bigoni F, Houck ML, Ryder OA, Wienberg J, Stanyon R. 2004. Chromosome painting shows that Pygathrix nemaeus has the most basal karyotype among Asian colobines. Int J Primatol 25 (3):679-688.

Bleisch W, Xie JH. 1998. Ecology and behavior of the Guizhou snub-nosed langur (Rhinopithecus [Rhinopithecus] brelichi), with a discussion of socioecology in 
the genus. In: Jablonski NG, editor. The natural history of the doucs and snub-nosed monkeys. Singapore: World Scientific Publishing. p 217-239.

Boonratana R, Canh LX. 1998. Preliminary observations of the ecology and behavior of the Tonkin snub-nosed monkey (Rhinopithecus [Presbytiscus] avunculus) in Northern Vietnam. In: Jablonski NG, editor. The Natural History of the Doucs and Snub-nosed Monkeys. Singapore: World Scientific Publishing. p 207-215.

Brandon-Jones D. 1984. Colobus and leaf monkeys. In: MacDonald ID, editor. Encylopedia of Mammals. London: George Allen and Unwin. p:398-408.

Brandon-Jones D. 1995. A revision of the Asian pied leaf monkeys (Mammalia: Cercopithecidae: Superspecies Semnopithecus auratus), with a description of a new subspecies. Raffles Bull Zool 43:3-43.

Brandon-Jones D, Eudey AA, Geissmann T, Groves CP, Melnick DJ, Morales JC, Shekelle M, Stewart CB. 2004. Asian Primate Classification. Int J Primatol 25:97164.

Brunet M, Guy F, Pilbeam D, Lieberman DE, Likius A, Mackaye HT, Ponce de León MS, Zollokofer CP, Vignaud P. 2005. New material of the earliest hominid from the Upper Miocene of Chad. Nature 434:752-755.

Burnham KP, Anderson DR. 2002. Model Selection and Multimodel Inference: A Practical Information-Theoretic Approach. New York: Springer.

Castresana J. 2000. Selection of conserved blocks from multiple alignments for their use in phylogenetic analysis. Mol Biol Evol 17:540-552.

Chakraborty D, Ramakrishnan U, Panor J, Mishra C, Sinha A. 2007. Phylogenetic relationships and morphometric affinities of the Arunachal macaque Macaca munzala, a newly described primate from Arunachal Pradesh, northeastern India. Mol Phylogenet Evol 44:838-849.

Chan YC, Roos C, Inoue-Murayama M, Inoue E, Shih CC, Pei KJC, Vigilant L. 2010. Mitochondrial genome sequence effectively reveal the phylogeny of Hylobates gibbons. Plos One 5 (12):e14419.

Chaplin G, Jablonski NG. 1998. The integument of the odd-nosed colobines. In: Jablonski NG, editor. The natural history of the doucs and snub-nosed monkeys. Recent advances in human biology. Singapore and London: World Scientific Publishing Co. p 79-104. 
Chaves R, Sampaio I, Schneider MP, Schneider H, Page SL, Goodman M. 1999. The place of Callimico goeldii in the callithrichine phylogenetic tree: evidence from von Willebrand factor gene intron 11 sequences. Mol Phylogenet Evol 13:392404.

Collura RV, Auerbach MR, Stewart CB. 1996. A quick, direct method that can differentiate expressed mitochondrial genes from their nuclear pseudogenes. Curr Biol 6 (10): 1337-1339.

Cortés-Ortiz L, Duda TF Jr, Canales-Espinosa D, Garcia-Orduna F, RodriguezLuna E, Bermingham E. 2007. Hybridization in large-bodied New World primates. Genetics 176:2421-2425.

Covert HH, Le KQ, Wright BW. 2008. On the Brink of Extinction: research for the conservation of the Tonkin snub-nosed monkey (Rhinopithecus avunculus), In: Fleagle JG, editor. Elwyn Simons: A Search for Origins. New York: NY, Kluwer Press. p 409427.

Cui LW, Sheng AH, He XC, Xiao W. 2006. Birth seasonality and interbirth interval of captive Rhinopithecus bieti. Am J Primatol 68:457-463.

Darwin C.1859. On the origin of species by means of natural selection or the preservation of favoured races in the struggle of life. London: John Murray.

Davies AG, Oates JF. 1994. Colobine monkeys - their ecology, behaviour and evolution. Cambridge: Cambridge University Press.

de Jong G, de Ruiter JR, Haring R. 1994. Genetic structure of a population with social structure and migration. In: Loeschcke V, Tomiuk J, Jain SK, editors. Conservation genetics. Basel: Birkha"user Verlag. p 147-164.

Delson E. 1975. Evolutionary history of the Cercopithecidae. Contrib Primatol 5:167-217.

Delson E. 2000. Cercopithecinae. In: Delson E, Tattersall I, Van Couvering JA, Brooks AS, editors. Encyclopedia of human evolution and prehistory. New York: Garland Publishing Inc. p 166-171.

Dietz JM, Baker AJ, Ballou JD. 2000. Demographic evidence of inbreeding depression in wild golden lion tamarins. In: Yang A, Clark GM, editors. Genetics, demography, and viability of fragmented populations. Cambridge: Cambridge University Press. p 203-211. 
Di Fiore A. 2003. Molecular genetic approaches to the study of primate behavior, social organization, and reproduction. Am J Phys Anthropol 122:62-99.

Disotell TR, Honeycutt RL, Ruvolo M. 1992. Mitochondrial DNA phylogeny of the Old World Monkey Tribe Papionini. Mole Biol Evol 9(1):1-13.

Dobzhansky Th. 1973. Nothing in biology makes sense except in the light of evolution. Am Biol Teach 35:125-129.

Dong TH, Boonratana R. 2006. Further imformation on ecology and behavior of Tonkin snub-nosed monkey (Rhinopithecus avunculus) in Vietnam. Entebe, Uganda: paper presented at the the meeting of the international Primatological Society.

Drummond AJ, Ho SY, Phillips MJ, Rambaut A. 2006. Relaxed phylogenetics and dating with confidence. PLoS Biol 4:e88

Drummond AJ, Kearse M, Heled J, Moir R, Thierer T, Ashton B, Wilson A, Stones-Havas S. 2009. Geneious, version 4.6.1. http://www.geneious.com

Drummond AJ, Rambaut A. 2007. BEAST: Bayesian evolutionary analysis by sampling trees. BMC Evol Biol 7:214.

Dutrillaux B. 1979. Chromosomal evolution in Primate: tentative phylogeny from Microcebus murinus (Prosimians) to man. Hum Genet 48: 251-314.

Edwards AWF, Cavalli-Sforza LL. 1964. Reconstruction of evolutionary trees. In: Heywood VH, McNeill J, editors. Phenetic and Phylogenetic Classification. London: Systematics Association pub. p 67-76.

Ellerman JR, Morrison-Scott TCS. 1951. Checklist of Palaearctic and Indian mammals. London: Trustees of the British Museum (Natural History). p 22-89.

Ellstrand NC, Elam DR. 1993. Population genetic consequences of small population size: implications for plant conservation. Annu Rev Ecol Syst 24: 217-242.

Evans RC. 1999. Molecular, morphological, and ontogenetic evaluation of relationships and evolution in the Rosaceae. PhD diss. University of Toronto.

Excoffier L, Lischer HL. 2010. Arlequin suite ver 3.5: a new series of programs to perform population genetics analyses under Linux and Windows. Mol Ecol Res 10:564-567.

Excoffier L, Smouse PE, Quattro JM. 1992. Analysis of molecular variance inferred from metric distances among DNA haplotypes: application to human mitochondrial DNA restriction data. Genetics 131:479-491. 
Fabre V, Condemi S, Degioanni A. 2009. Genetic evidence of geographical groups among Neanderthals. PloS ONE 4 (4): e5151.

Falk D. 2000. Primate diversity. New York: W.W. Norton and Company.

Fashing PJ. 2011. African colobine monkeys: Their behavior, ecology, and conservation. In: Campbell C, Fuentes A, MacKinnon K, Bearder S, Stumpf R, editors. Primates in Perspective. Oxford: Oxford University Press. p 203-229.

Fox CW, Wolf JB. 2006. Evolutionary genetics: concepts and case studies. New York: Oxford University Press.

Frankel OH, Soule ME. 1981. Conservation and Evolution. Cambridge: Cambridge University Press.

Frankham R. 1995. Conservation genetics. Annu Rev Genet 29:305-327

Frankham R, Ballou JD, Briscoe DA. 2004. A primer of conservation genetics. Cambridge: Cambridge University Press.

Frost SR, Marcus L, Bookstein F, Reddy C, Delson E. 2003. Cranial allometry, phylogeography, and systematics of large-bodied papionins (Primates: Cercopithecinae) inferred from geometric morphometric analysis of landmark data. Anat. Rec. 275A: 1048-1072.

Fu YX. 1997. Statistical tests of neutrality of mutations against population growth, hitchhiking and background selection. Genetics 147:915-925.

Funk D, Omland K. 2003. Species-level paraphyly and polyphyly: frequency, causes, and consequences, with insights from animal mitochondrial DNA. Annu Rev Ecol Evol Syst 34:397-423.

Geissmann T, Lwin N, Aung SS, Aung TN, Aung MZ, Hla TH, Grindler M, Momberg F. 2011. A new species of snub-nosed monkey, genus Rhinopithecus MilneEdwards, 1872 (Primates, Colobinae), from northern Kachin State, northeastern Myanmar. Am J Primatol 73:96-107.

Gilbert LB, Kasuga T, Glass NL, Taylor JW. 2011. Array CGH phylogeny: how accurate are comparative genomic hybridization-based trees? BMC Genomics 12:487501.

Goodman M, Porter CA, Czelusniak J, Page SL, Schneider H, Shoshani J, Gunnell G, Groves CP. 1998. Toward a phylogenetic classification of primates based on DNA evidence complemented by fossil evidence. Mol Phylogenet Evol 9:585-598. 
Grechko V. 2002. Using Molecular DNA Markers in Phylogeny and Systematics. Russ J Genet 38:1013-1033.

Green RE, Krause J, Briggs AW, Maricic T, Stenzel U, Kircher M, Patterson N, Li $H$, Zhai W, Fritz MH, Hansen NF, Durand EY, Malaspinas AS, Jensen JD, MarquesBonet T, Alkan C, Prüfer K, Meyer M, Burbano HA, Good JM, Schultz R, Aximu-Petri A, Butthof A, Höber B, Höffner B, Siegemund M, Weihmann A, Nusbaum C, Lander ES, Russ C, Novod N, Affourtit J, Egholm M, Verna C, Rudan P, Brajkovic D, Kucan Z, Gusic I, Doronichev VB, Golovanova LV, Lalueza-Fox C, de la Rasilla M, Fortea J, Rosas A, Schmitz RW, Johnson PL, Eichler EE, Falush D, Birney E, Mullikin JC, Slatkin M, Nielsen R, Kelso J, Lachmann M, Reich D, Pääbo S. 2010. A draft sequence of the Neandertal genome. Science 328:710-722.

Groves CP. 1970. The forgotten Leaf-Eaters and the phyologeny of the Colobinae. In: Napier JR, Napier PH, editors. Old World Monkeys. New York: Academic Press p 555-587.

Groves CP. 1978. Phylogenetic and population systematics of the mangabeys (Primates: Cercopithecoidea). Primates 19:1-34.

Groves CP. 1987. A theory of human and primate evolution. Oxford: Oxford University Press.

Groves CP. 1989. A Theory of Human and Primate Evolution. Oxford: Clarendon Press.

Groves CP. 2001. Primate taxonomy. Washington, DC: Smithsonian Institution Press.

Groves CP. 2007. The taxonomic diversity of the Colobinae of Africa. J Anthropol Sci 85: 7-34.

Grueter CC, Li D, Ren B Wei F, van Schaik CP. 2009. Dietary profile of Rhinopithecus bieti and its socioecological implications. Int J Primatol 30:601-624.

Grubb P, Butynski TM, Oates JF, Bearder SK, Disotell TR, Groves CP. Struhsaker TT. 2003. Assessment of the diversity of African primates. Int J Primatol 24 (6):1301-1357.

Hall TA. 1999. BioEdit: a user-friendly biological sequence alignment editor and analysis program for Windows 95/98/NT. Nucleic Acids Symp 41:95-98.

Hallet B, Molnar P. 2001. Distorted drainage basins as markers of crustal strain east of the Himalayas. J Geophys Res 106:13697-13709. 
Hamilton MB.2009. Populaiton genetics. Unite Kingdom: John Wiley \& Sons Ltd.

Harris EE, Disotell TR. 1998. Nuclear gene trees and the phylogenetic relationships of the mangabeys (Priamtes: Papionini). Mol Biol Evol 15:892-900.

Harpending HC. 1994. Signature of ancient population growth in a low-resolution mitochondrial DNA mismatch distribution. Hum Biol 66:591-600.

Hartl DL, Clark AG. 2007. Principles of population genetics. Sunderland, MA: Sinauer.

Hartwig WC, 2002. The primate fossil record. United Kingdom: Cambridge University Press.

Harvey PH, Leigh Brown AJ, Maynard SJ, Nee S. 1996. New Uses for New Phylogenies. New York: Oxford University Press.

Hayaishi S, Kawamoto Y. 2006. Low genetic diversity and biased distribution of mitochondrial DNA haplotypes in the Japanese macaque (Macaca fuscata yakui) on Yakushima Island. Primates 47:158-164.

Hellborg L, Ellegren H. 2003. Y chromsome conserved anchored tagged sequences (YCATS) for the analysis of mammalian male-specific DNA. Mol Ecol 12:283-291.

Hennig W. 1965. Phylogenetic systematic. Annu. Rev. Entomol 10:97-116.

Herke SW, Xing J, Ray DA, Zimmermann JW, Cordaux R, Batzer MA. 2007. A SINEbased dichotomous key for primate identification. Gene 390:39-51.

Hill WCO. 1934. A monography on the purple-faced leaf-monkeys (Pithecus vetulus). Ceylon J Sci 9:23-88.

Hill WCO. 1952. The external and visceral anatomy of the Olive colobus monkey (Procolobus verus). J Zool 122 (1):127-186.

Hill WCO. 1974. Primates: Comparative Anatomy and Taxonomy, Cynopithecinae (Cercocebus, Macaca, Cynopithecus). Edinburgh: Edinburgh University Press.

Höglund J. 2009. Evolutionary conservation genetics. Oxford: Oxford University Press.

Huelsenbeck JP, Ronquist F, Nielsen R, Bollback JP. 2001. Bayesian inference of phylogeny and its impact on evolutionary biology. Science 294:2310-2314.

IUCN. 2011. The IUCN red list of threatened species. Available at: http://www.iucnredlist.org. 
Jablonski NG. 1992. Dental agenesis as evidence of possible genetic differences isolation in the colobine monkey, Rhinopithecus roxellana. Primates 33: 371-376.

Jablonski NG. 1998a. The response of catarrhine primates to Pleistocene environmental fluctuations in East Asia. Primates 39:29-37.

Jablonski NG. 1998b. The evolution of the doucs and snubnosed monkeys and the question of the phyletic unity of the odd-nosed colobines. In: Jablonski NG, editor. The natural history of the doucs and snub-nosed monkeys. Singapore: World Scientific Publishing. p 13-52.

Jablonski NG.1998c. Natural History of the Doucs and Snub-Nosed Monkeys. New Jersey: World Scientific Publishing Company.

Jablonski NG. 1999. Primate evolution - in and out of Africa. Curr Biol 9:R119,R122.

Jablonski NG, Peng YZ. 1993. The phylogenetic relationships and classification of the doucs and snub-nosed langurs of China and Vietnam. Folia Primatol 60:36-55.

Ji WZ, Zou RJ, Shang EY, Zhou HW, Yang SC, Tian BP. 1998. Maintenance and breeding of Yunnan snub-nosed monkeys (Rhinopithecus [Rhinopithecus] bieti) in captivity. In: Jablonski NG, editor. The natural history of the doucs and snubnosed monkeys. Singapore: World Scientific Publishing. p 323-335.

Johnson AD, Drum M, Bachvarova RF, Masi T, White ME, Crother BI. 2003. Evotution of predetermined germ cells in vertebrate embryos: implications for macroevolution. Evol Dev 5 (4):414-431.

Jolly CJ. 1966. Introduction to the Cercopithecoidea, with notes on their use as laboratory animals. Symp Zool Soc London 17:427-457.

Jolly CJ, Whitehead PF. 2000. Old world monkeys: three decades of development and change in the study of the Cercopithecoidea. In: Whitehead PF, Jolly CJ, editors, Old world monkeys. Unite Kingdom: Cambridge University Press. p 1-28.

Karanth KP, Singh L, Collura RV, Stewart CB. 2008. Molecular phylogeny and biogeography of langurs and leaf monkeys of South Asia (Primates: Colobinae). Mol Phylogenet Evol 46:683-694.

Karanth KP. 2010. Molecular systematic and conservation of the langurs and leaf monkeys of South Asia. J Genet 89 (4):393-399.

Katoh K, Kuma K, Toh H, Miyata T. 2005. MAFFT version 5: improvement in accuracy of multiple sequence alignment. Nucleic Acids Res 33:511-518. 
Kazazian HH. 2004. Mobile elements: drivers of genome evolution. Science 303 (5664):1626-1632.

Kelley J. 2002. The hominoid radiation in Asia. In: Hartwig WC, editor. The Primate Fossil Record. Cambridge: Cambridge University Press. p 369-384.

Kingdon J. 1997. The Kingdon Field Guide to African Mammals. San Diego, California, USA: Academic Press Natural World.

Kirkpatrick RC. 1995. The natural history and conservation of the snub-nosed monkeys (genus Rhinopithecus). Biol Conserv 72(3):363-9.

Kirkpatrick RC. 2011. The Asian colobines: Diversity among leaf-eating monkeys. In: Campbell C, Fuentes A, MacKinnon K, Bearder S, Stumpf R, editors. Primates in Perspective. Oxford: Oxford University Press. p 189-202.

Kishino H, Hasegawa M. 1989. Evaluation of the maximum likelihood estimate of the evolutionary tree topologies from DNA sequence data, and the branching order in Hominoidea. J Mol Evol 29:170-179.

Koblmüller S, Duftner N, Sefc KM, Aibara M, Stipacek M, Blanc M, Egger B, Sturmbauer C. 2007. Reticulate phylogeny of gastropod-shell-breeding cichlids from Lake Tanganyika - the result of repeated introgressive hybridization. BMC Evol Biol $7: 7$.

Kubatko LS, Carstens BC, Knowles LL. 2009. STEM: species tree estimation using maximum likelihood for gene trees under coalescence. Bioinformatics 25:971973.

Kuhn HJ. 1972. On the perineal organ of male Procolobus badius. J Hum Evol 1:371-378.

Lande R, Shannon S. 1996. The role of genetic variation in adaptation and population persistence in a changing environment. Evolution 50: 434-437.

Leakey MG. 1993. Evolution of Theropithecus in the Turkana Basin. In: Jablonski NG, editor. Theropithecus, the Rise and Fall of a Primate Genus. Cambridge: Cambridge University Press. p 85-124.

Lebatard $A E$, Bourlès $D L$, Duringer $P$, Jolivet $M$, Braucher $R$, Carcaillet $J$, Schuster M, Arnaud N, Monié P, Lihoreau F, Likius A, Mackaye HT, Vignaud P, Brunet M. 2008. Cosmogenic nuclide dating of Sahelanthropus tchadensis and Australopithecus bahrelghazali: Mio-Pliocene hominids from Chad. Proc Natl Acad Sci USA 105:3226-3231. 
Li BG, Pan RL, Oxnard CE. 2002. Extinction of snub-nosed monkeys in China during the past 400 years. Int J Primatol 23:1227-1244.

Li HP, Meng SJ, Men ZM, Fu YX, Zhang YP. 2003. Genetic diversity and population history of golden monkeys (Rhinopithecus roxellana). Genetics 164:269275.

Li J, Han K, Xing J, Kim HS, Rogers J, Ryder OA, Disotell T, Yue B, Batzer MA. 2009. Phylogeny of macaques (Cercopithecidae: Macaca) based on Alu elements. Gene 448:242-249.

Li M, Liang B, Feng ZJ, Tamate HB. 2001. Molecular phylogenetic relationships among Sichuan snub-nosed monkeys (Rhinopithecus roxellana) inferred from mitochondrial cytochrome b gene sequences. Primates 42:153-160.

Li M, Liu ZJ, Gou JX, Gou JX, Ren BP, Pan RL, Su YJ, Funk SM, Wei FW. 2007. Phylogeography and population structure of the golden monkeys (Rhinopithecus roxellana): inferred from mitochondrial DNA sequences. Am J Primatol 69:1195-1209.

Li Y, Wang X, Ting N, Zhang YP. 2011. Mitogenomic analysis of Chinese snubnosed monkeys: Evidence of positive selection in $\mathrm{NADH}$ dehydrogenase genes in highaltitude adaptation. Mitochondrion 11:497-503.

Li M, Wei FW, Huang CM, Pan RL, de Ruiter J. 2004. Phylogeny of snub-nosed monkeys inferred from mitochondrial DNA, cytochrome $b$, and 12S rRNA sequences. Int J Primatol 25:861-873.

Liang B, Qi HJ, Zhang SY, Ren BP. 2001. Developmental traits of captive Sichuan snub-nosed monkeys (Rhinopithecus roxellana) at different age stages. Acta Zool Sinica 47:381-387.

Librado P, Rozas J. 2009. DnaSP version 5: a software for comprehensive analysis of DNA polymorphism data. Bioinformatics 25:1451-1452.

Liu WT, Huang CM, Roos C, Zhou QH, Li YB, Wei FW. 2008. Identification of the species, origin and sex of smuggled douc langur (Pygathrix sp.) remains. Vietnamese $\mathrm{J}$ Primatol 2: 63-69.

Liu ZJ, Ren BP, Wei FW, Long YC, Hao YL, Li M. 2007. Phylogeography and population structure of the Yunnan snubnosed monkey (Rhinopithecus bieti) inferred from mitochondrial control region DNA sequence analysis. Mol Ecol 16:3334-3349.

Liu ZJ, Ren BP, Wu RD, Zhao L, Hao YL, Wang BS, Wei FW, Long YC, Li M. 2009. The effect of landscape features on population genetic structure in Yunnan snub- 
nosed monkeys (Rhinopithecus bieti) implies an anthropogenic genetic discontinuity. Mol Ecol 18:3831-3846.

Losos JB. 2011. Convergence, adaptation, and constraint. Evolution 65 (7):1827-1840.

MacKinnon J. 2008. Order primates. In: Smith AT, Xie Y, editors. A guide to the mammals of China. Princeton: Princeton University Press. p 158-172.

Maddison WP, Maddison DR. 2009. Mesquite: a modular system for evolutionary analysis, version 2.6. http://mesquite.org

Mayr E. 1963. Animal species and evolution. Cambridge, MA: Belknap Press.

McCracken K, Sorenson M. 2005. Is homoplasy or lineage sorting the source of incongruent mtDNA and nuclear gene trees in the stiff-tailed ducks (NomonyxOxyyura)? Syst Biol 54:35-55.

McGraw WS, Fleagle JG. 2000. Biogeography and evolution of the Cercocebus Mandrillus clade. Am J Phys Anthro Supp 30:225.

Md-Zain BM, Lee SJ, Lakim M, Ampeng A, Mahani MC. 2010. Phylogenetic position of Tarsius bancanus based on partial Cytochrome b DNA sequences. J. Biol Sci 10 (4):348-354.

Meijaard E, Groves CP. 2006. The geography of mammals and rivers in mainland Southeast Asia. In: Lehman SM, Fleagle JG, editors. Primate Biogeography. New York: Springer. p 305-329.

Merker S, Driller C, Perwitasari-Farajallah D, Pamungkas J, Zischler H. 2009. Elucidating geological and biological processes underlying the diversification of Sulawesi tarsiers. Proc Natl Acad Sci USA 106:8459-8464.

Messier W, Stewart CB. 1997. Episodic adaptive evolution of primate lysozymes. Nature 385:151-154.

Meyer D, Rinaldi ID, Ramlee H, Perwitasari-Farajallah D, Hodges JK, Roos C. 2011. Mitochondrial phylogeny of leaf monkeys (genus Presbytis, Eschscholtz, 1821) with implications for taxonomy and conservation. Mol Phylogenet Evol 59:311-319.

Miller KG, Kominz MA, Browning JV, Wright JD, Mountain GS, Katz ME, Sugarman PJ, Cramer BS, Christie-Blick N, Pekar SF. 2005. The phanerozoic record of global sea-level change. Science 310:1293-1298.

Mittermeier RA, Wallis J, Rylands AB, Ganzhorn JU, Oates JF, Williamson EA, Palacios E, Heymann EW, Kierulff MCM, Long Y, Supriatna J, Roos C, Walker S, 
Corte's-Ortiz L, Schwitzer C. 2009. Primates in peril: the world's 25 most endangered primates 2008-2010. J Primate Conserv 24:1-57.

Modolo L, Salzburger W, Martin RD. 2005. Phylogeography of Barbary macaques (Macaca sylvanus) and the origin of the Gibraltar colony. Proc Natl Acad Sci USA 102:7392-7397.

Morales JC, Melnick DJ. 1998. Phylogenetic relationships of the macaques (Cercopithecoida: Macaca), as revealed by high resolution restriction site mapping of mitochondrial ribosomal genes. J Hum Evol 34:1-23.

Morin PA, Chambers KE, Boesch C, Vigilant L. 2001. Quantitative polymerase chain reaction analysis of DNA from noninvasive samples for accurate microsatellite genotyping of wild chimpanzees (Pan troglodytes verus). Mol Ecol 10:1835-1844.

Moulin S, Gerbault-Seureau M, Dutrilluaux B, Richard FA. 2008. Phylogenomics of African guenons. Chromosome Res 16 (5): 783-99.

Nadler T, Momberg F, Nguyen XD, Lormée N. 2003. Vietnam Primate Conservation Status Review 2002. Part 2: Leaf Monkeys. Hanoi: Frankfurt Zoological Society and Fauna \& Flora Internatioan-Indochina Programme.

Napier JR, Napier PH. 1967. A handbook of living primates. London: Academic Press.

Napier PH. 1985. Catalogue of primates in the British Museum (Natural History) and elsewhere in the British Isles. Part III: Family Cercopithecidae, subfamily Colobinae. London, British Museum (Natural History).

Nei M. 1975. Molecular population genetics and evolution. New York: American Elsevier.

Nei M. 1977. F-statistics and analysis of gene diversity in subdivided populations. Ann Hum Genet 41:225-233.

Nei M. 1987. Molecular evolutionary genetics. New York: Columbia University Press.

Nei M, Li WH. 1979. Mathematical model for studying genetic variation in terms of restriction endonucleases. Proc Natl Acad Sci. USA 76:5269-5273.

Newton PN, Dunbar RIM. 1994. Colobine monkey society. In: Davies AG, Oates JF, editors. Colobine monkeys: their ecology, behaviour and evolution. Cambridge: Cambridge University Press p 311e346. 
Nichols R. 2001. Gene trees and species trees are not the same. Trends Ecol Evol 16:358-364.

Nguyen N. 2000. A survey of Tonkin snub-nosed monkeys (Rhinopithecus avunculus) in Northern Vietnam. Folia Primatol 71:157-160.

Oates JF. 1994. The natural history of African colobines. In: Davies AG, Oates JF, editors. Colobine Monkeys: Their Ecology, Behaviour and Evolution. Cambridge: Cambridge University Press. p 75-128.

Oates JF, Davies AG, Delson E. 1994. The diversity of living colobines. In: Davies AG, Oates JF, editors. Colobine monkeys: their ecology, behavior and evolution. Cambridge: Cambridge University Press.

Okada N. 1991. SINEs. Curr Opin Genet Dev 1:498-504.

Osterholz M, Walter L, Roos C. 2008. Phylogenetic position of the langur genera Semnopithecus and Trachypithecus among Asian colobines, and genus affiliations of their species groups. BMC Evol Biol 8:58.

Osterholz M, Walter L, Roos C. 2009. Retropositional events consolidate the branching order among New World monkey genera. Mol Phylogenet Evol 50:507-513.

Page SL, Chiu C, Goodman M. 1999. Molecular phylogeny of Old World monkeys (Cercopithecidae) as inferred from y-globin DNA sequences. Mol Phylogenet Evol 13:348-359.

Pan YR. 1995. Fossil primates discovered in China. In: Xia W, Zhang Y, editors. Primate Research and Conservation. Beijing: China Forestry Publishing House. p 99_ 105.

Pan D, Hu HX, Meng SJ, Men ZM, Fu YX, Zhang YP. 2009. A high polymorphism level in Rhinopithecus roxellana. Int J Primatol 30:337-351.

Pan YR, Jablonski NG. 1987. The age and geographical distribution of fossil cercopithecids in China. Hum Evol 2: 59-69.

Pan RL, Oxnard CE. 2001. Cranial morphology of the golden monkey (Rhinopithecus) and douc langur (Pygathrix nemaeus). Hum Evol 16:199-223.

Patterson N, Richter DJ, Gnerre S, Lander ES, Reich D. 2006. Genetic evidence for complex speciation of humans and chimpanzees. Nature 441:1103-1108.

Pääbo S. 2003. The mosaic that is our genome. Nature 421:409-412.

Peakall R, Smouse PE. 2006. GENALEX 6: genetic analysis in Excel: population genetic software for teaching and research. Mol Ecol Notes 6:1288-1295. 
Peng YZ, Pan RL, Jablonski N. 1993. Classification and evolution of Asian colobines. Folia Primatol 60:106-117.

Peng YZ, Ye ZZ, Zhang YP, Pan RL. 1988. The classification and phylogeny of snub-nosed monkey Rhinopithecus spp. based on gross morphological characters. Zool Res 9:239-248.

Perelman P, Johnson WE, Roos C, Seuảnez HN, Horvath JE, Moreira MAM, Kessing B, Pontius J, Roelke M, Rumpler Y, Schneider MPC, Silva A, O'Brien SJ, Pecon-Slattery J. 2011. Am molecular phylogeny of living primates. PLoS Genet 7:e1001342.

Petit RJ, Excoffier L. 2009. Gene flow and species delimitation. Trends Ecol Evol 4:386-393.

Philippe H, Laurent J. 1998. How good are deep phylogenetic trees? Curr Opin Genetics Dev 8:616-623.

Pocock RI. 1935. The monkeys of the generae Pithecus (Presbytis) and Pygathrix found to the east of the Bay of Bengal. Proc Zool Soc 104:1895-1961.

Posada D. 2008. jModelTest: Phylogenetic model averaging. Mol Biol Evol 25:1253-1256.

Pollard DA, lyer VN, Moses AM, Eisen MB. 2006. Widespread discordance of gene trees with species tree in Drosophila: evidence for incomplete lineage sorting. PLoS Genet 2:e173.

Posada D, Crandall KA. 1998. Modeltest: testing the model of DNA substitution. Bioinformatics 14:817-818.

Posada D. 2008. Model Test: phylogenetic model averaging. Mol Biol Evol 25:1253-1256.

Pusey AE, Packer C. 1987. Dispersal and philopatry. In: Smuts BB, Cheney DL, Seyfarth RM, Wrangham RW, Struhsaker TT, editors. Primate Societies. Chicago: University of Chicago Press. p 250-266.

Qi XG, Li BG, Ji WH. 2008. Reproductive parameters of wild female Rhinopithecus roxellana. Am J Primatol 70:311-319.

Quan GQ, Xie JH. 1981. Notes on Rhinopithecus roxellanae brelichi (Thomas). Acta Theriol Sinica 1:113-116.

Quan GQ, Xie JY. 2002. Research on the golden monkey. Shanghai: Science and Education Publishing House. 
Raaum RL, Sterner KN, Noviello CM, Stewart CB, Disotell TR. 2005. Catarrhine primate divergence dates estimated from complete mitochondrial genomes: concordance with fossil and nuclear DNA evidence. J Hum Evol 48:237-257.

Rambaut A. 2008. FigTree: Tree figure drawing tool, version 1.2.2. Available at: http://tree.bio.ed.ac.uk/software/figtree/.

Rambaut A, Drummond AJ. 2007. Tracer: MCMC trace analysis tool, version 1.4.1. Available at: http://tree.bio.ed.ac.uk/software/ tracer/.

Ray DA, Xing J, Salem AH, Batzer MA. 2006. SINEs of a nearly perfect character. Syst Biol 55:928-935. Ray DA, Xing J, Hedges DJ Hall MA, Laborde ME, Anders BA, White BR, Stoilova N, Fowlkes JD, Landry KE, Chemnick LG, Ryder OA, Batzer MA. 2005. Alu insertion loci and platyrrhine primate phylogeny. Mol Phylogenet Evol 35:117-126.

Reichenbach HGL. 1862. Die Vollständige Naturgeschichte der Affen. In Die Vollständige Naturgeschichte des in- und Auslandes Dresden. Dresden and Leipzig, Germany: Central-Atlas für Zoologische Gärten.

Ren B, Li M, Long Y, Grüter CC, Wei F. 2008. Measuring daily ranging distances of Rhinopithecus bieti via a Global Positioning System collar at Jinsichang, China: a methodological consideration. Int J Primatol 29:783-794.

Rogers AR, Harpending H. 1992. Population growth makes waves in the distribution of pairwise genetic differences. Mol Biol Evol 9:552-569.

Ronquist F, Huelsenbeck JP. 2003. MrBayes 3: Bayesian phylogenetic inference under mixed models. Bioinformatics 19: 1572-1574.

Roos C, Nadler T. 2001. Molecular evolution of the douc langurs. Zool Garten NF 71:1-6.

Roos C, Ziegler T, Hodges K, Zischler H, Abegg C. 2003. Molecular phylogeny of Mentawai macaques: taxonomic and biogeographic implications. Mol Phylogenet Evol 29:139-150.

Roos C. 2004. Molecular evolution and systematics of Vietnamese primates. In: Nadler T, Streicher U, Ha Thang Long, editors. Conservation of primates in Vietnam. Hanoi: Haki Publishing. p 23-28.

Roos C, Schmitz J, Zischler H. 2004. Primate jumping genes elucidate strepsirrhine phylogeny. Proc Natl Acad Sci USA 101:10650-10654. 
Roos C, Thanh VN, Walter L, Nadler T. 2007. Molecular systematic of Indochinese primates. Vietn J Primatol 1:41-53.

Roos C, Nadler T, Walter L.2008. Mitochondrial phylogeny, taxonomy and biogeography of the silvered langur species group (Trachypithecus cristatus). Mol Phylogenet Evol 47 (2):629-636.

Rowe N. 1996. The pictorial guide to the living primates. East Hampton: Pogonias Press.

Rumpler Y, Warter S, Hauwy M, Fausser JL, Roos C, Zinner D. 2008. Comparing chromosomal and mitochondrial phylogenies of sportive lemurs (genus Lepilemur, Primates). Chromosome Res 16:1143-1158.

Salem AH, Ray DA, Xing J, Callinan PA, Myers JS, Hedges DJ, Garber RK, Witherspoon DJ, Jorde LB, Batzer MA. 2003. Alu elements and hominid phylogenetics. Proc Natl Acad Sci USA 22:12787-12791.

Salem AH, Ray DA, Batzer MA. 2005. Identity by descent and DNA sequence variation of human SINE and LINE elements. Cytogenet Genome Res 108:63-72.

Santos C, Montiel R, Sierra B, Bettencourt C, Fernandez E, Alvarez L, Lima M, Abade A, Aluja MP. 2005. Understanding differences between phylogenetic and pedigree-derived mtDNA mutation rate: a model using families from the Azores islands (Portugal). Mol Biol Evol 22:1490-1505.

Savage JM. 1997. Society of Systematic Biologists Presidential Address. Boulder: University of Colorado.

Seehausen O. 2004. Hybridization and adaptive radiation. Trends Ecol Evol 19:198-207.

Schmidt D, Pool J. 2002. The effect of population history on the distribution of the Tajima's D statistic. New York: Cornell University Press.

Schmitz J, Ohme M, Zischler H. 2001. SINE insertions in cladistic analyses and the phylogenetic affiliations of Tarsius bancanus to other primates. Genetics 157:777784.

Schmitz J, Roos C, Zischler H. 2005. Primate phylogeny: molecular evidence from retroposons. Cytogenet Genome Res 108:26-37.

Schneider S, Excoffier L. 1999. Estimation of demographic parameters from the distribution of pairwise differences when the mutation rates vary among sites: application to human mitochondrial DNA. Genetics 152:1079-1089. 
Shedlock AM, Okada N. 2000. SINE insertions: powerful tools for molecular systematics. Bioessays 22:148-160.

Simberloff D. 1988. The contribution of population and community biology to conservation science. Annu Rev Ecol Syst 19:473-511.

Shimodaira H, Hasegawa M. 1999. Multiple comparisons of log-likelihoods with applications to phylogenetic inference. Mol Biol Evol 16:1114-1116.

Soares P, Ermini L, Thomson N, Mormina M, Rito T, Ro“hl A, Salas A, Oppenheimer S, Macaulay V, Richards MB. 2009. Correcting for purifying selection: an improved human mitochondrial molecular clock. Am J Hum Genet 84:740-759.

Soltis PS, Soltis DE. 2003. Applying the bootstrap in phylogeny reconstruction. Statistical Sci 18 (2):256-267.

Spielman D, Brook BW, Frankham R. 2004. Most species are not driven to extinction before genetic factors impact them. Proc Natl Acad Sci USA 101:1526115264.

Stearns SC, Hoekatry RF. 2000. Evolution: an introdeuction. New York: Oxford University Press.

Sterner KR, Raaum RL, Zhang YP, Stewart CB, Disotell TR. 2006. Mitochondrial data support an odd-nosed colobine clade. Mol Phylogenet Evol 40:1-7.

Stewart CB, Disotell TR. 1998. Primate evolution - in and out of Africa. Curr Biol 8:R582-R588.

Stewart CB, Disotell TR. 1999. Primate evolution - in and out of Africa. Curr Biol 9:R120-R122.

Strasser E, Delson E. 1987. Cladistic analysis of cercopithecid relationships. J Hum Evol 16:81-99.

Swofford, DL. 2003. PAUP*: phylogenetic analysis using parsimony (* and other methods), version 4.0b10. Sunderland, MA: Sinauer.

Struhsaker TT. 1981. Census methods for estimating densities. In: Struhsaker TT, editor. Techniques for the Study of Primate Population Ecology. Washington DC: National Academy Press. p 36-80.

Szalay FS, Delson E. 1979. Evolutionary history of the primates. New York: Academic Press.

Tajima F. 1989. Statistical method for testing the neutral mutation hypothesis by DNA polymorphism. Genetics 123:585-595. 
Tan C, Guo S, Li B. 2007. Population structure and ranging patterns of Rhinopithecus roxellana in Zhouzhi National Nature Reserve, Shaanxi, China. Int J Primatol 28:577-591.

Thalmann O, Fischer A, Lankester F, Pääbo S, Vigilant L. 2007. The complex evolutionary history of gorillas: insights from genomic data. Mol Biol Evol 24:146-158.

Thinh VN, Mootnick AR, Geissmann T, Li M, Ziegler T, Agil M, Moisson P, Nadler T, Walter L, Roos C. 2010. Mitochondrial evidence for multiple radiations in the evolutionary history of small apes. BMC Evol Biol 10:74.

Ting N. 2005. Mitochondrial relationships and divergence dates of the African colobines: evidence of Miocene origins for the living colobus monkeys. J Hum Evol 55:312-325.

Ting N. 2008. Mitochondrial relationships and divergence dates of the African colobines: evidence of Miocene origins for the living colobus monkeys. J Hum Evol $55: 312-325$.

Ting N, Tosi AJ, Li Y, Zhang YP, Disotell TR. 2008. Phylogenetic incongruence between nuclear and mitochondrial markers in the Asian colobines and the evolution of the langurs and leaf monkeys. Mol Phylogenet Evol 46:466-474.

Tosi AJ, Detwiler KM, Disotell TR. 2003. X-chromosomal window into the evolutionary history of the guenons (Primates: Cercopithecini). Mol Phylogenet Evol 36:58-66.

Tosi AJ, Melnick DJ, Disotell TR. 2004. Sex chromosome phylogenetics indicate a single transition to terrestriality in the guenons (tribe Cercopithecini). J Hum Evol 46 (2), 223-237.

Tosi AJ, Detwiler KM, Disotell TR. 2005. Y-chromosomal markers suitable for non-invasive studies of guenon hybridization. Int J Primatol 36:58-66.

Usher MB. 1997 . Small populations: Fragmentation, population dynamics and population genetics. In: Tew TE, Crawford TJ, Spencer JW, Stevens DP, Usher MB, Warren J, editors. The role of genetics in conserving small populations. Unite Kingdom: Joint Nature Conservation Committee, Peterborough. p $11-21$.

Van de Lagemaat LN, Gagnier L, Medstrand P, Mager DL. 2005. Genomic deletions and precise removal of transposable elements mediated by short identical DNA segments in primates. Genome Res 15:1243-1249. 
Van Oosterhout C, Hutchinson WF, Wills DPM, Shipley P. 2004. Micro-Checker: software for identifying and correcting genotyping errors in microsatellite data. Mol Ecol Notes 4:535-538.

Verheyen WN. 1962. Contribution a la craniologie comparee des Primates: les genres Colobus Illiger 1811 et Cercopithecus Linne. 1758. Ann. Mus. Roy. de l'Afrique Centrale, Ser. in $8^{\circ}$, Sci Zool 105:1-255.

Vignaud $\mathrm{P}$, Duringer $\mathrm{P}$, Mackaye HT, Likius A, Blondel C, Boisserie JR, De Bonis L, Eisenmann V, Etienne ME, Geraads D, Guy F, Lehmann T, Lihoreau F, Lopez-Martinez N, Mourer-Chauviré C, Otero O, Rage JC, Schuster M, Viriot L, Zazzo A, Brunet M. 2002. Geology and palaeontology of the Upper Miocene Toros-Menalla hominid locality, Chad. Nature 418:152-155.

Vogel C. 1976. Ecology, lifestyle and social behavior of langur groups in differing Indian habitats. Fort der Verhal Tensf 17: 1-160.

Wakeley J. 1997. Using the variance of pairwise differences to estimate the recombination rate. Genet Res 69:45-48.

Waits LP, Luikart G, Taberlet P. 2001. Estimating the probability of identity among genotypes in natural populations: cautions and guidelines. Mol Ecol 10:249256.

Wang W, Forstner MRJ, Zhang YP, Liu ZM, Wei Y, Huang HQ, Hu HG, Xie YX, Wu DH, Melnick DJ. 1997. A phylogeny of Chinese leaf monkeys using mitochondrial ND3-ND4 gene sequences. Int J Primatol 18:305-320.

Weitzel V, Yang CM, Groves CP. 1988. A catalogue of primates in the Singapore Zoological Reference Collection. Raffles Bull Zool 36:1-166.

Whelan S, Lió P, Goldman N. 2001. Molecular phylogenetics: state-of-the-art methods for looking into the past. Trends in Genetics 17 (5):262-272

Whittaker DJ, Ting N, Melnick DJ. 2006. Molecular phylogenetic affinities of the simakobu monkey (Simias concolor). Mol Phylogenet Evol 39:887-892.

Whitfield LS, Lovell-Badge R, Goodfellow PN. 1993. Rapid sequence evolution of the mammalian sex-determining gene SRY. Nature 364:713-715.

Whybrow PJ. 1992. Land movements and species dispersal. In: Jones S, Martin RE, Pilbeam D, editors. The Cambridge Encyclopedia of Human Evolution. Cambridge: Cambridge University Press. p 169-173. 
Wiley EQD, Siegel-Causey DRB, Funk DVA. 1991. The compleat cladist. Univ Kans Mus Nat Hist Spec Publ 19:1-158.

Winney BJ, Hammond RL, Macasero W, Flores B, Boug A, Biquand V, Biquand S, Bruford MW. 2004. Crossing the Red Sea: phylogeography of the hamadryas baboon, Papio hamadryas hamadryas. Mol Ecol 13:2819-2827.

Wright S. 1969. Evolution and the genetics of populations. II: The theory of gene frequencies. Chicago: University of Chicago Press.

Wright S. 1978. Evolution and the genetics of populations. IV: the variability within and among natural populations. Chicago: University of Chicago Press.

Wu G, Wang HC, Fu HW, Zhao JZ, Yang YQ. 2004. Habitat selection of Guizhou golden monkey (Rhinopithecus roxellanae brelichi) in Fanjing Mountain Biosphere Reserve, China. J Forest Res 15:197-202.

Wyman SK, Jansen RK, Boore JL. 2004. Automatic annotation of organellar genomes with DOGMA. Bioinformatics 20:3252-3255.

Xing J, Wang H, Han K, Ray DA, Huang CH, Chemnick LG, Stewart CB, Disotell TR, Ryder OA, Batzer MA. 2005. A mobile element based phylogeny of Old World monkeys. Mol Phylogenet Evol 37:872-880.

Xing J, Wang H, Zhang Y, Ray DA, Tosi AJ, Disotell TR, Batzer MA. 2007. A mobile element based evolutionary history of guenons (Tribe Cercopithecini). BMC Biol $5: 5$.

Xing J, Witherspoon DJ, Ray DA, Batzer MA, Jorde LB. 2007. Mobile DNA elements in primate and human evolution. Am J Phys Anthropol 45(Suppl):2-19.

Yang M, Sun D, Zinner D, Roos C. 2009. Reproductive parameters in Guizhou snub-nosed monkeys (Rhinopithecus brelichi). Am J Primatol 71:266-270.

Yang YQ, Lei XP, Sun DY, Yang CD, He RT, Zhang WY. 2002. Ecology of the wild Guizhou snub-nosed monkey. Guiyang: Guizhou Scientific Institute Press.

Yang Z. 2002. Likelihood and Bayes estimation of ancestral population sizes in hominoids using data from multiple loci. Genetics 162:1811-1823.

Young NM, MacLatchy L. 2004.The phylogenetic position of Moropithecus. J Hum Evol 46:163-184.

Zalmout IS, Sanders WJ, MacLatchy LM, Gunnell GF, Al-Mufarreh YA, Ali MA, Nasser AAH, Al-Masari AM, Al-Sobhi SA, Nadhra AO, Matari AH, Wilson JA, Gingerich 
PD. 2010. New Oligocene primate from Saudi Arabia and the divergence of apes and Old World monkeys. Nature 466:360-365.

Zhang SY, Liang B, Wang LX. 2000. Seasonality of mating and births in captive Sichuan golden monkeys (Rhinopithecus roxellanae). Am J Primatol 51:265-269.

Zhang YP, Ryder OA, Fan Z, Zhang H. 1997. Sequence variation and genetic diversity in giant panda. Sci China C Life Sci 27:139-144.

Zhang YP, Ryder OA. 1998. Mitochondrial cytochrome b gene sequences of Old World monkeys: with a special reference on evolution of Asian colobines. Primates 39:39-49.

Zhang YP, Ryder OA. 1999. Primate evolution - in and out of Africa. Curr Biol 9:R119-R120.

Zhang YZ, Quan GQ, Zhao TG, Southwick CH. 1992. Distribution of primates (except Macaca) in China. Acta Theriol Sinica 12:81-95.

Zhang P, Watanabe K, Li B. 2008. Female social dynamics in a provisioned freeranging band of the Sichuan snub-nosed monkey (Rhinopithecus roxellana) in the Qinling mountains, China. Am J Primatol 70:1013-1022.

Ziegler T, Abegg C, Meijaard E, Perwitasari-Farajallah D, Walter L, Hodges JK, Roos C. 2007. Molecular phylogeny and evolutionary history of Southeast Asian macaques forming the M. silenus group. Mol Phyl Evol 42:807-816.

Zinner D, Arnold ML, Roos C. 2009. Is the new primate genus Rungwecebus a baboon? PLoS ONE 4:e4859.

Zinner D, Arnold ML, Roos C (in press). The strange blood: natural hybridization in primates. Evol Anthropol

Zinner D, Groeneveld LF, Keller C, Roos C. 2009. Mitochondrial phylogeography of baboons (Papio spp.) - indication for introgressive hybridization? BMC Evol Biol 9:83.

Zwickl DJ. 2006. Genetic algorithm approaches for the phylogenetic analysis of large biological sequence datasets under the maximum-likelihood criterion. PhD thesis. The University of Texas at Austin. 


\section{Curriculum Vitae}

PERSONAL

Date of Birth: January 22, 1973

Status: Married

Gender: Female

Nationality: P.R. China

\section{EDUCATION}

2007 - Present

Ph.D. in Biological Sciences - Georg-AugustUniversity Göttingen, Germany

Thesis:

Evolutionary History of Snub-nosed Monkeys (Rhinopithecus spp.) and Population Genetic Structure of the Guizhou Snub-nosed Monkey ( $R$. brelichi)

Area of Specialization: Phylogenetics and Population Genetics

Advisor:

Prof. Dr. Peter M. Kappeler

$1994-1997$

M.S. in Medicine Sciences - Guiyang Medical College, Guizhou, China,

Thesis:

The Analysis of Physiological and Biochemical Index in Guizhou Snub-nosed Monkey (R. brelichi)

Advisor: Prof. Hanbin Chen

$1990-1994$

B.A. in Biological Sciences - South-West Forestry University, Yunnan, China. 


\section{Publications}

Yang MY, Roos C, Nadler T, Zinner D. in press. The evolutionary history of Oddnosed monkeys. In: Tan CL, Grueter C, Wright B, editors. Odd-nosed monkeys: Recent advances in the study of the forgotten Colobines. Primatology: Progress and Prospects series.

Roos C, Zinner D, Kubatko LS, Schwarz C, Yang MY, Meyer D, Dash SD, Xing JC, Batzer MA, Brameier M, Leendertz FH, Ziegler T, Perwitasari-Farajallah D, Nadler T, Walter L, Osterholz M. 2011. Nuclear versus mitochondrial DNA: evidence for hybridization in Colobine monkeys. BMC Evolutionary Biology 11: 1-13. DOI: 10.1186/1471-2148-11-77.

Yang MY, Yang YQ, Cui DY, Fickenscher G, Roos C, Brameier M. 2011. Population genetic structure of Guizhou snub-nosed monkeys (Rhinopithecus brelichi) as inferred from mitochondrial control region sequences, and comparison with R.bieti and R.roxellana. Am J Physi Anthropol. DOI: 10.1002/ajpa.21618

Yang MY, Sun DY, Zinner D, Roos C. 2009. Reproductive parameters in Guizhou snub-nosed monkeys (Rhinopithecus brelichi). Am J Primatol 71 (3): 266 270.

Yang MY, Pyörnilä A, Meyer-Rochow BV. 2004. UV-reflectivity of parafocal eyespot elements on butterfly wings in normal and abnormal specimens. Entomol Fennica 15 (1):34 - 40.

Yang MY, Meyer-Rochow BV. 2004. Fine-structural details of the photoreceptor membranes of the ocellus of the scale-insect parasite Centrodora sp. (Hymenoptera, Apheneilidae): A case of gene transfer between host and parasite? Biocell 28 (2):151154.

Meyer-Rochow BV, Yang MY. 2004. Not just an empty cavity: the interrhabdomal space in the Jamaican cave fly Neoditomyia farri (Diptera, Mycetophilidae). Zool Sci 21:719-722.

Yang MY, Cheng HB. 2002. To determine and analysis E2 and TEST of Guizhou Golden Monkey by using radioimmunoassay. Guizhou Science 20 (3):56-58. (in Chinese) 
Yang MY, Lei XP, Qiu Y. 1998. Test and analysis eight microelements from hair of wild and artificial breeding Guizhou Golden Monkey. J Guiyang Medical College 11(2):67-69. (in Chinese)

Wu FL, Tao BY, Yang MY, Sun DY. 1997. Test of T3, T4 and TSH blood concentration: compare and analysis the relation among Guizhou Golden Monkey, Yunnan Golden Monkey and Sichuan Golden Monkey. J Guiyang Medical College 22 (2):193-194. (in Chinese)

Wu FL, Tao BY, Yang MY, Sun DY. 1997. The analysis of serum estradiol content of Guizhou snub-nosed monkeys. Guizhou Sci 15 (2):118-120. (in Chinese)

Yang MY, Wu FL, Tao BY, Sun DY. 1996. To determine and analysis blood coagulation factor of Guizhou Golden Monkey. Guizhou Sci 14 (4):29-32. (in Chinese) 


\section{Statement of Authorship}

Except where reference is made in the text of this dissertation, this dissertation contains no material published elsewhere or extracted in whole or in part from a dissertation presented by me for another degree or diploma. No other person's work has been used without due acknowledgement in the main text of the dissertation. This dissertation has not been submitted for the award of any other degree or diploma in any other tertiary institution.

All sentences or passages quoted in this dissertation from other people's work have been specifically acknowledged by clear cross-referencing to author, work and page(s). Any illustrations which are not the work of the author of this dissertation have been used with the explicit permission of the originator and are specifically acknowledged.

I understand that failure to do this amounts to plagiarism and will be considered grounds for failure in this dissertation and the degree examination as a whole.

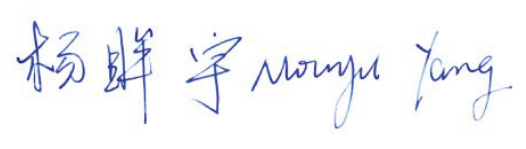

Mouyu Yang

Bad Homburg, November 2011 